\title{
Wave turbulence in one-dimensional models
}

\author{
V.E. Zakharov ${ }^{\mathrm{a}, \mathrm{b}}$, P. Guyenne ${ }^{\mathrm{c}}$, A.N. Pushkarev ${ }^{\mathrm{d}}$, F. Dias ${ }^{\mathrm{e}, *}$ \\ a Landau Institute for Theoretical Physics, Moscow, Russia \\ ${ }^{\mathrm{b}}$ Department of Mathematics, University of Arizona, Tucson, AZ 85721, USA \\ c Institut Non-Linéaire de Nice, France \\ ${ }^{\mathrm{d}}$ Waves and Solitons LLC, 738 W. Sereno Dr., Gilbert, AZ, USA \\ e Centre de Mathematiques et de Leurs Applications, Ecole Normale Supérieure de Cachan, \\ 61 Avenue du Président Wilson, 94235 Cachan Cedex, France
}

\begin{abstract}
A two-parameter nonlinear dispersive wave equation proposed by Majda, McLaughlin and Tabak is studied analytically and numerically as a model for the study of wave turbulence in one-dimensional systems. Our ultimate goal is to test the validity of weak turbulence theory. Although weak turbulence theory is independent on the sign of the nonlinearity of the model, the numerical results show a strong dependence on the sign of the nonlinearity. A possible explanation for this discrepancy is the strong influence of coherent structures - wave collapses and quasisolitons — in wave turbulence. (C) 2001 Published by Elsevier Science B.V.
\end{abstract}

Keywords: Weak turbulence; Wave collapses; Quasisolitons; Kinetic wave equation; Kolmogorov spectra

\section{Introduction}

A wide variety of physical problems involve random nonlinear dispersive waves. The most common tool for the statistical description of these waves is a kinetic equation for squared wave amplitudes, the so-called kinetic wave equation. Sometimes this equation is also called Boltzmann's equation. This terminology is in fact misleading because the kinetic wave equation and Boltzmann's equation are the opposite limiting cases of a more general kinetic equation for particles which obey Bose-Einstein statistics like photons in stellar atmospheres or phonons in liquid helium. It was first derived by Peierls in 1929 [1]. In spite of the fact that both the kinetic wave equation and Boltzmann's equation can be derived from the quantum kinetic equation, the kinetic wave equation was derived independently and almost simultaneously in plasma physics and for surface waves on deep water. This was done in the early 1960s while Boltzmann's equation was derived in the 19th century! The derivation for surface waves is due to Hasselmann [2,3] (see also Zakharov [4]).

Once the kinetic wave equation has been derived, the shape of wave number spectra can be predicted by the so-called weak turbulence (WT) theory. It is called weak because it deals with resonant interactions between small-amplitude waves. Thus, contrary to fully developed turbulence, it leads to explicit analytical solutions provided some assumptions are made. So far, there have been only a few studies to check the results of WT the-

\footnotetext{
* Corresponding author.

E-mail address: dias@cmla.ens-cachan.fr (F. Dias).
} 
ory. Recently, Pushkarev and Zakharov [5] numerically solved the three-dimensional dynamical equations for the free-surface elevation and the velocity potential in the case of capillary water waves. They obtained an isotropic spectrum close to the theoretical power-law found by Zakharov and Filonenko [6]. Majda, McLaughlin and Tabak [7] (hereafter referred to as MMT) considered four-wave interactions by introducing a one-dimensional model equation. This equation can be integrated numerically quite efficiently on large inertial intervals. They examined a family of Kolmogorov-type solutions depending on the parameters of the equation. The validity of several theoretical hypotheses was then assessed numerically. Namely, MMT confirmed the random phase and quasi-Gaussian approximations. They also showed the independence of the solutions on the nature of forces, initial conditions, and the size and level of discreteness of the computational domain. However, their simulations surprisingly displayed spectra steeper than the predicted ones. They explained the discrepancy by proposing a new inertial range scaling technique which seems to yield the appropriate exponents. More recently, Cai et al. [8-10] revisited their earlier results and found some results which agree with WT theory as well. ${ }^{1}$ They considered two kinds of Hamiltonians: Hamiltonians which are the sum of a quadratic term and a quartic term (positive nonlinearity), as in [7], and Hamiltonians which are the difference between a quadratic and a quartic term (negative nonlinearity). For either sign of nonlinearity, they found agreement with MMT theory in some cases and agreement with WT theory in some other cases. Since their computations were performed with a dispersion relation in which the frequency varies like the square root of the wave number, one can see an analogy with deep water waves. Incidentally, the WT theory was recently developed for shallow water waves by Zakharov [11].

As in many other fields, numerical modeling leads to some difficulties, especially when one wants to compare with the theory. Most of these difficulties are related to finite-size effects, i.e. the domain is discretized into a grid of points in computations whereas one assumes an infinite medium in theory. We can mention the bottleneck phenomenon [12] which tends to flatten the slope of the inertial range at small scales. It is commonly observed in problems with a dissipative cutoff. In addition, Pushkarev [13] revealed the phenomenon of frozen turbulence at very low levels of nonlinearity. In this situation, the resonance conditions have very few solutions (or may not be fulfilled at all!) because of the discrete values of wave numbers. As a consequence, there is no energy flux due to the lack of resonating wave vectors. The power-law regime only takes place at moderate levels of nonlinearity where quasi-resonant interactions come into play. Pushkarev concluded that WT in bounded systems combines the features of both frozen and Kolmogorov-type turbulence. The beauty of the MMT model equation is that the above mentioned difficulties can be controlled completely.

After introducing the model equation, the paper is divided into two parts. In the first part (Sections 3-11), the MMT equation is studied analytically. A WT description of the equation is provided (see [7]). We find the Kolmogorov solutions of the kinetic equation and determine the set of parameters for which such solutions can be realized. Then we discuss the coherent structures which can compete with WT. The most simple coherent structures are solitons similar to the soliton solutions of the nonlinear Schrödinger equation (NLS).

Solitons for the MMT equation exist only if nonlinearity is negative. In the cases of interest, they are shown to be unstable (see Section 7) and cannot play an important role in the wave dynamics.

As an alternative to soliton coherent structures, there are wave collapses described by self-similar solutions of the MMT equation. These solutions can exist in a certain parameter regime for both signs of nonlinearity. Theoretically speaking, both solitons and collapses can coexist with WT.

Another type of coherent structures are quasisolitons, or envelope solitons. They were discussed recently by Zakharov and Kuznetsov [14]. In the MMT model quasisolitons exist at positive nonlinearity only. Their stability remains an open question.

\footnotetext{
${ }^{1}$ These three papers were kindly given to us when the present manuscript was essentially completed. Some of the results are similar to ours, but their interpretation is different.
} 
The main new theoretical results of the first part are a careful tabulation of the signs of the fluxes for the MMT model equation, the existence and possible role of quasisolitons for positive nonlinearity, and an analogy with Phillips spectrum associated with the formation of collapses.

In the second part (Sections 12-14), we describe the results of the numerical study of the MMT equation. We find that the wave turbulence described by the MMT equation is different both quantitatively and qualitatively for both signs of nonlinearity. Since the predictions of WT theory are identical for both signs of nonlinearity, WT theory can be applied at best for one sign of nonlinearity. Our analysis of the results leads to somewhat contradictory results.

For positive nonlinearity the balance of energy and particle fluxes as well as the level of turbulence are in good agreement with WT theory. Meanwhile the slope of the spectrum in the window of transparency is steeper than predicted by WT theory.

In the case of negative nonlinearity the picture of turbulence is quite different from the WT predictions, both qualitatively and quantitatively. First of all, the turbulence is stabilized on a level which is one order of magnitude less than predicted by WT theory. Then the sign of the flux of particles is opposite to the one predicted by WT theory. Both these facts lead to a conjecture on the existence of a strong and essentially nonlinear mechanism which competes successfully with WT quartic resonances. In our opinion, this mechanism is the wave collapse, described by self-similar solutions of the MMT equations. At the same time, the high-frequency tail of the spectrum has a slope which coincides exactly with the slope predicted by WT theory. This leads to the conclusion that in spite of the presence of wave collapses, the high-frequency asymptotics of spectra is governed by the WT processes which are responsible for carrying only a small part of the energy. The coexistence of wave collapses and WT was already described in the context of the 2D NLS [15].

Wave collapse is an example of an essentially nonlinear coherent structure arising in wave turbulence under certain conditions. As said above, another important type of coherent structures are quasisolitons or envelope solitons living for a finite time. Such structures can arise in the MMT model in the case of positive nonlinearity. We believe that these structures are responsible for the deviation of the spectra from the ones predicted by WT theory.

\section{Model equation}

We investigate the family of dynamical equations

$$
\mathrm{i} \frac{\partial \psi}{\partial t}=\left|\frac{\partial}{\partial x}\right|^{\alpha} \psi+\lambda\left|\frac{\partial}{\partial x}\right|^{\beta / 4}\left(\left.\left.|| \frac{\partial}{\partial x}\right|^{\beta / 4} \psi\right|^{2}\left|\frac{\partial}{\partial x}\right|^{\beta / 4} \psi\right), \quad \lambda= \pm 1,
$$

where $\psi(x, t)$ denotes a complex wave field and $\alpha, \beta$ are real parameters.

If $\lambda=+1$, one exactly recovers the MMT model which was treated in [7]. Note that our parameter $\beta$ is the opposite of the parameter $\beta$ in MMT. The extension $\lambda= \pm 1$ in Eq. (2.1), which was also treated in [8-10], raises an interesting problem because the balance between nonlinear and dispersive effects may change according to $\lambda$.

Besides the Hamiltonian

$$
H=H_{\mathrm{L}}+H_{\mathrm{NL}}=\int\left(\left.\left.|| \frac{\partial}{\partial x}\right|^{\alpha / 2} \psi\right|^{2}+\left.\left.\frac{\lambda}{2}|| \frac{\partial}{\partial x}\right|^{\beta / 4} \psi\right|^{4}\right) \mathrm{d} x,
$$

the system (2.1) preserves two other integrals of motion: wave action and momentum, respectively

$$
N=\int|\psi|^{2} \mathrm{~d} x \quad \text { and } \quad M=\frac{\mathrm{i}}{2} \int\left(\psi \frac{\partial \psi^{*}}{\partial x}-\frac{\partial \psi}{\partial x} \psi^{*}\right) \mathrm{d} x .
$$


As usual, it is convenient to work in Fourier space. Let us write Eq. (2.1) as

$$
\mathrm{i} \frac{\partial \hat{\psi}_{k}}{\partial t}=\omega(k) \hat{\psi}_{k}+\int T_{123 k} \hat{\psi}_{1} \hat{\psi}_{2} \hat{\psi}_{3}^{*} \delta\left(k_{1}+k_{2}-k_{3}-k\right) \mathrm{d} k_{1} \mathrm{~d} k_{2} \mathrm{~d} k_{3},
$$

where $\hat{\psi}_{k}=\hat{\psi}(k, t)$ denotes the $k$ th component in the Fourier decomposition of $\psi(x, t)$ and $(*)$ stands for complex conjugation.

In this form, Eq. (2.3) looks like the so-called one-dimensional Zakharov's equation determined by the linear dispersion relation

$$
\omega(k)=|k|^{\alpha}, \quad \alpha>0,
$$

and the simple interaction coefficient

$$
T_{123 k}=T\left(k_{1}, k_{2}, k_{3}, k\right)=\lambda\left|k_{1} k_{2} k_{3} k\right|^{\beta / 4} .
$$

One easily sees that the kernel $T_{123 k}$ possesses the symmetry required by the Hamiltonian property

$$
T_{123 k}=T_{213 k}=T_{12 k 3}=T_{3 k 12} .
$$

Moreover, the absolute values in Eqs. (2.4) and (2.5) ensure the basic assumptions of isotropy and scale invariance. In other words, $\omega(k)$ and $T_{123 k}$ are invariant with respect to rotations $(k \rightarrow-k)$ and they are homogeneous functions of their arguments with degrees $\alpha$ and $\beta$, respectively, i.e.

$$
T\left(\xi k_{1}, \xi k_{2}, \xi k_{3}, \xi k\right)=\xi^{\beta} T\left(k_{1}, k_{2}, k_{3}, k\right), \quad \xi>0 .
$$

Following MMT, we fix $\alpha=\frac{1}{2}$ by analogy with gravity waves whose dispersion relation reads as $\omega(k)=(g k)^{1 / 2}$ ( $g$ being the acceleration due to gravity). The power $\beta$ takes the value +3 if the analogy is extended to the nonlinear term but we will consider a wider range of values for $\beta$.

Eq. (2.3) describes four-wave interaction processes obeying the resonant conditions

$$
\begin{aligned}
& k_{1}+k_{2}=k_{3}+k, \\
& \omega_{1}+\omega_{2}=\omega_{3}+\omega .
\end{aligned}
$$

For $\alpha>1$ these equations only have the trivial solution $k_{3}=k_{1}, k=k_{2}$ or $k_{3}=k_{2}, k=k_{1}$. For $\alpha<1$ there is also a non-trivial solution. Note that in this case the signs of $k_{i}$ must be different. For instance, $k_{1}<0$ and $k_{2}, k_{3}, k>0$. If $\alpha=\frac{1}{2}$, Eqs. (2.8) and (2.9) can be parameterized by two parameters $A$ and $\xi$

$$
k_{1}=-A^{2} \xi^{2}, \quad k_{2}=A^{2}\left(1+\xi+\xi^{2}\right)^{2}, \quad k_{3}=A^{2}(1+\xi)^{2}, \quad k=A^{2} \xi^{2}(1+\xi)^{2} .
$$

In the case $\alpha=2$ and $\beta=0$, Eq. (2.1) becomes the NLS equation

$$
\mathrm{i} \frac{\partial \psi}{\partial t}=-\frac{\partial^{2} \psi}{\partial x^{2}}+\lambda|\psi|^{2} \psi
$$

(note here that $|\partial / \partial x|^{2}=-\partial^{2} / \partial x^{2}$ ).

Positive nonlinearity $\lambda=+1$ corresponds to the defocusing NLS, while negative nonlinearity corresponds to the focusing NLS. 


\section{Weak turbulence description of the model equation}

If one only considers small nonlinear effects, then the statistical behavior can be mainly described by the evolution of the two-point correlation function

$$
\left\langle\hat{\psi}(k, t) \hat{\psi}^{*}\left(k^{\prime}, t\right)\right\rangle=n(k, t) \delta\left(k-k^{\prime}\right),
$$

where brackets denote ensemble averaging. We introduce also the four-wave correlation function

$$
\left\langle\hat{\psi}\left(k_{1}, t\right) \hat{\psi}\left(k_{2}, t\right) \hat{\psi}^{*}\left(k_{3}, t\right) \hat{\psi}^{*}(k, t)\right\rangle=J_{123 k} \delta\left(k_{1}+k_{2}-k_{3}-k\right) .
$$

On this basis, WT theory leads to the kinetic equation for $n(k, t)$ and provides tools for finding stationary power-law solutions (for details, see [7]). Here we explain the main steps of the procedure applied to our model.

The starting point is the original equation for $n(k, t)$. From Eq. (2.3), we have

$$
\frac{\partial n_{k}}{\partial t}=2 \int \operatorname{Im} J_{123 k} T_{123 k} \delta\left(k_{1}+k_{2}-k_{3}-k\right) \mathrm{d} k_{1} \mathrm{~d} k_{2} \mathrm{~d} k_{3} .
$$

Due to the quasi-Gaussian random phase approximation

$$
\operatorname{Re} J_{123 k} \simeq n_{1} n_{2}\left[\delta\left(k_{1}-k_{3}\right)+\delta\left(k_{1}-k\right)\right] .
$$

The imaginary part of $J_{123 k}$ can be found through an approximate solution of the equation imposed on this correlator. The result is (see [16])

$$
\operatorname{Im} J_{123 k} \simeq 2 \pi T_{123 k}^{*} \delta\left(\omega_{1}+\omega_{2}-\omega_{3}-\omega\right)\left(n_{1} n_{2} n_{3}+n_{1} n_{2} n_{k}-n_{1} n_{3} n_{k}-n_{2} n_{3} n_{k}\right) .
$$

This gives

$$
\begin{aligned}
\frac{\partial n_{k}}{\partial t}= & 4 \pi \int\left|T_{123 k}\right|^{2}\left(n_{1} n_{2} n_{3}+n_{1} n_{2} n_{k}-n_{1} n_{3} n_{k}-n_{2} n_{3} n_{k}\right) \delta\left(\omega_{1}+\omega_{2}-\omega_{3}-\omega\right) \\
& \times \delta\left(k_{1}+k_{2}-k_{3}-k\right) \mathrm{d} k_{1} \mathrm{~d} k_{2} \mathrm{~d} k_{3} .
\end{aligned}
$$

Since the square norm cancels the sign of $T_{123 k}$, it is clear that the WT approach is independent on $\lambda$. Here we point out that MMT mistakenly wrote a factor $12 \pi$ instead of $4 \pi$ in Eq. (3.5) and the right-hand side of Eq. (3.5) with the opposite sign. This fact is particularly important when determining the fluxes of wave action and energy.

Assuming that $n(-k)=n(k)$ (similarly to an angle averaging in higher dimensions), one gets

$$
\begin{aligned}
\frac{\partial \mathcal{N}(\omega)}{\partial t}= & \frac{4 \pi}{\alpha^{4}} \int\left(\omega_{1} \omega_{2} \omega_{3} \omega\right)^{(\beta / 2-\alpha+1) / \alpha}\left(n_{1} n_{2} n_{3}+n_{1} n_{2} n_{\omega}-n_{1} n_{3} n_{\omega}-n_{2} n_{3} n_{\omega}\right) \delta\left(\omega_{1}+\omega_{2}-\omega_{3}-\omega\right) \\
& \times\left[\delta\left(\omega_{1}^{1 / \alpha}+\omega_{2}^{1 / \alpha}-\omega_{3}^{1 / \alpha}+\omega^{1 / \alpha}\right)+\delta\left(\omega_{1}^{1 / \alpha}+\omega_{2}^{1 / \alpha}+\omega_{3}^{1 / \alpha}-\omega^{1 / \alpha}\right)\right. \\
& \left.+\delta\left(\omega_{1}^{1 / \alpha}-\omega_{2}^{1 / \alpha}-\omega_{3}^{1 / \alpha}-\omega^{1 / \alpha}\right)+\delta\left(-\omega_{1}^{1 / \alpha}+\omega_{2}^{1 / \alpha}-\omega_{3}^{1 / \alpha}-\omega^{1 / \alpha}\right)\right] \mathrm{d} \omega_{1} \mathrm{~d} \omega_{2} \mathrm{~d} \omega_{3}
\end{aligned}
$$

where $\mathcal{N}(\omega)=n(k(\omega)) \mathrm{d} k / \mathrm{d} \omega, n_{\omega}$ stands for $n(k(\omega))$ and $\omega$ is given by Eq. (2.4).

The next step consists in inserting the power-law ansatz

$$
n(\omega) \propto \omega^{-\gamma},
$$

and then performing the Zakharov's conformal transformations $[7,15,16]$. Finally, the kinetic equation becomes

$$
\frac{\partial \mathcal{N}(\omega)}{\partial t} \propto \omega^{-y-1} I(\alpha, \beta, \gamma)
$$


where

$$
\begin{aligned}
I(\alpha, \beta, \gamma)= & \frac{4 \pi}{\alpha^{4}} \int_{\Delta}\left(\xi_{1} \xi_{2} \xi_{3}\right)^{((\beta / 2+1) / \alpha)-1-\gamma}\left(1+\xi_{3}^{\gamma}-\xi_{1}^{\gamma}-\xi_{2}^{\gamma}\right) \delta\left(1+\xi_{3}-\xi_{1}-\xi_{2}\right) \\
& \times \delta\left(\xi_{1}^{1 / \alpha}+\xi_{2}^{1 / \alpha}+\xi_{3}^{1 / \alpha}-1\right)\left(1+\xi_{3}^{y}-\xi_{1}^{y}-\xi_{2}^{y}\right) \mathrm{d} \xi_{1} \mathrm{~d} \xi_{2} \mathrm{~d} \xi_{3}
\end{aligned}
$$

with

$$
\Delta=\left\{0<\xi_{1}<1,0<\xi_{2}<1, \xi_{1}+\xi_{2}>1\right\} \text { and } y=3 \gamma+1-\frac{2 \beta+3}{\alpha} .
$$

The non-dimensionalized integral $I(\alpha, \beta, \gamma)$ is obtained by using the change of variables $\omega_{j} \rightarrow \omega \xi_{j}(j=1,2,3)$.

The ansatz (3.7) makes sense if the integral in (3.6) converges. It could diverge both at low and high frequencies. The condition of convergence at low frequencies coincides with the condition of convergence of the integral in (3.9) and can be easily found. It reads

$$
2 \gamma<-1+\frac{\beta+4}{\alpha}
$$

The condition of convergence at high frequencies can be found after substituting (3.7) into (3.6). Omitting the details, we get the result

$$
\gamma>\frac{\beta+\alpha-1}{\alpha}
$$

In all the cases discussed in this paper, both conditions (3.10) and (3.11) are satisfied.

For the case $\alpha=\frac{1}{2}$, one can transform Eq. (3.6) into the form

$$
\frac{\partial \mathcal{N}(\omega)}{\partial t}=64 \pi \omega^{4(\beta+1)}\left[S_{1}+S_{2}+S_{3}+S_{4}\right]
$$

where

$$
\begin{aligned}
S_{1}= & \int_{0}^{1} \frac{u^{2 \beta+2}(u+1)^{2 \beta+1}}{\left(1+u+u^{2}\right)^{3 \beta+4}}\left[n\left(\frac{u}{1+u+u^{2}} \omega\right) n\left(\frac{u(u+1)}{1+u+u^{2}} \omega\right) n\left(\frac{u+1}{1+u+u^{2}} \omega\right)\right. \\
& +n(\omega) n\left(\frac{u(u+1)}{1+u+u^{2}} \omega\right) n\left(\frac{u+1}{1+u+u^{2}} \omega\right)-n(\omega) n\left(\frac{u}{1+u+u^{2}} \omega\right) n\left(\frac{u+1}{1+u+u^{2}} \omega\right) \\
& \left.-n(\omega) n\left(\frac{u}{1+u+u^{2}} \omega\right) n\left(\frac{u(u+1)}{1+u+u^{2}} \omega\right)\right] \mathrm{d} u \\
S_{2}= & \int_{0}^{1} \frac{\left(1+u+u^{2}\right)^{\beta+1}(u+1)^{2 \beta+1}}{u^{2 \beta+3}}\left[n\left(\frac{1+u+u^{2}}{u} \omega\right) n((u+1) \omega) n\left(\frac{u+1}{u} \omega\right)\right. \\
& +n(\omega) n((u+1) \omega) n\left(\frac{u+1}{u} \omega\right)-n(\omega) n\left(\frac{1+u+u^{2}}{u} \omega\right) n\left(\frac{u+1}{u} \omega\right) \\
& \left.-n(\omega) n\left(\frac{1+u+u^{2}}{u} \omega\right) n((u+1) \omega)\right] \mathrm{d} u, \\
S_{3}= & \int_{0}^{1} \frac{u^{2 \beta+2}\left(1+u+u^{2}\right)^{\beta+1}}{(1+u)^{2 \beta+3}}\left[n(u \omega) n\left(\frac{1+u+u^{2}}{1+u} \omega\right) n\left(\frac{u}{1+u} \omega\right)\right. \\
& \left.+n(\omega) n\left(\frac{1+u+u^{2}}{1+u} \omega\right) n\left(\frac{u}{1+u} \omega\right)-n(\omega) n(u \omega) n\left(\frac{1+u+u^{2}}{1+u} \omega\right)-n(\omega) n(u \omega) n\left(\frac{u}{1+u} \omega\right)\right] \mathrm{d} u,
\end{aligned}
$$




$$
\begin{aligned}
S_{4}= & \int_{0}^{1} \frac{\left(1+u+u^{2}\right)^{\beta+1}}{u^{2 \beta+3}(1+u)^{2 \beta+2}}\left[n\left(\frac{\omega}{u}\right) n\left(\frac{1+u+u^{2}}{u(1+u)} \omega\right) n\left(\frac{\omega}{1+u} \omega\right)+n(\omega) n\left(\frac{1+u+u^{2}}{u(1+u)} \omega\right) n\left(\frac{\omega}{u}\right)\right. \\
& \left.-n(\omega) n\left(\frac{\omega}{u}\right) n\left(\frac{\omega}{1+u}\right)-n(\omega) n\left(\frac{\omega}{u} \omega\right) n\left(\frac{1+u+u^{2}}{u(1+u)} \omega\right)\right] \mathrm{d} u .
\end{aligned}
$$

This equation can be used for the numerical simulation of WT.

\section{Kolmogorov solutions}

The aim is to look for stationary solutions of the kinetic equation. From Eqs. (3.8) and (3.9) we easily find that the stationarity condition

$$
\frac{\partial \mathcal{N}(\omega)}{\partial t}=0 \Leftrightarrow I(\alpha, \beta, \gamma)=0
$$

is satisfied only for $\gamma=0,1$ and $y=0,1$.

In terms borrowed from statistical mechanics, the cases $\gamma=0,1$ represent the thermodynamic equilibrium solutions

$$
n(\omega)=c
$$

where $c$ is an arbitrary constant and

$$
n(\omega) \propto \omega^{-1} \propto|k|^{-\alpha},
$$

which stem from the more general Rayleigh-Jeans distribution

$$
n_{\mathrm{RJ}}(\omega)=\frac{c_{1}}{c_{2}+\omega} .
$$

They correspond respectively to equipartition of particle number $N$ and quadratic energy $E$

$$
\begin{aligned}
& N=\int n(k) \mathrm{d} k=\int \mathcal{N}(\omega) \mathrm{d} \omega \\
& E=\int \omega(k) n(k) \mathrm{d} k=\int \omega \mathcal{N}(\omega) \mathrm{d} \omega
\end{aligned}
$$

The cases $y=0,1$ give the non-equilibrium Kolmogorov-type solutions, respectively

$$
n(\omega) \propto \omega^{(-2 \beta / 3-1+\alpha / 3) / \alpha} \propto|k|^{-2 \beta / 3-1+\alpha / 3},
$$

and

$$
n(\omega) \propto \omega^{-(2 \beta / 3+1) / \alpha} \propto|k|^{-2 \beta / 3-1},
$$

which exhibit typical dependence on the parameter $\beta$ of the interaction coefficient. The latter solutions are more interesting since realistic sea spectra are of Kolmogorov-type by analogy.

For the case $\alpha=\frac{1}{2}$ and $\beta=0$, the Kolmogorov-type solutions are

$$
\begin{aligned}
& n(\omega) \propto \omega^{-5 / 3} \propto|k|^{-5 / 6}, \\
& n(\omega) \propto \omega^{-2} \propto|k|^{-1} .
\end{aligned}
$$

Both exponents satisfy the conditions of locality (3.10) and (3.11). 


\section{Nature and sign of the fluxes}

The stationary non-equilibrium states are related to fluxes of integrals of motion, namely the quantities $N$ and $E$ in our four-wave interaction problem. We define the flux of particles (or wave action) and energy as, respectively

$$
\begin{aligned}
& Q(\omega)=-\int_{0}^{\omega} \frac{\partial \mathcal{N}\left(\omega^{\prime}\right)}{\partial t} \mathrm{~d} \omega^{\prime}, \\
& P(\omega)=-\int_{0}^{\omega} \omega^{\prime} \frac{\partial \mathcal{N}\left(\omega^{\prime}\right)}{\partial t} \mathrm{~d} \omega^{\prime} .
\end{aligned}
$$

Here, Eq. (4.7), respectively Eq. (4.8), is associated with a constant mean flux $Q_{0}$, respectively $P_{0}$, of particles, respectively energy. Let us now mention a physical argument which plays a crucial role in deciding the realizability of the Kolmogorov-type spectra. A more detailed justification is provided in Section 11 (see also [7,16]). Suppose that pumping is performed at some frequencies around $\omega=\omega_{f}$ and damping at $\omega$ near zero and $\omega_{\gg} \gg$. Weak turbulence theory then states that the energy is expected to flow from $\omega_{f}$ to higher $\omega$ 's (direct cascade with $P_{0}>0$ ) while the particles mainly head for lower $\omega$ 's (inverse cascade with $Q_{0}<0$ ). Accordingly, we need to evaluate the fluxes in order to select, among the rich family of power laws (4.7) and (4.8), those which are likely to result from numerical simulations of Eq. (2.1) with damping and forcing.

By inserting Eq. (3.8) into Eqs. (5.1) and (5.2), we obtain

$$
Q_{0} \propto \lim _{y \rightarrow 0} \frac{\omega^{-y}}{y} I, \quad P_{0} \propto \lim _{y \rightarrow 1} \frac{\omega^{-y+1}}{1-y} I
$$

which become

$$
\left.Q_{0} \propto \frac{\partial I}{\partial y}\right|_{y=0},\left.\quad P_{0} \propto \frac{\partial I}{\partial y}\right|_{y=1} .
$$

Using Eq. (3.9), the derivatives in Eq. (5.4) can be expressed as

$$
\begin{aligned}
-\left.\frac{\partial I}{\partial y}\right|_{y=0}= & \int_{\Delta} S\left(\xi_{1}, \xi_{2}, \xi_{3}\right)\left(1+\xi_{3}^{\gamma}-\xi_{1}^{\gamma}-\xi_{2}^{\gamma}\right) \delta\left(1+\xi_{3}-\xi_{1}-\xi_{2}\right) \\
& \times \ln \left(\frac{\xi_{1} \xi_{2}}{\xi_{3}}\right) \delta\left(\xi_{1}^{1 / \alpha}+\xi_{2}^{1 / \alpha}+\xi_{3}^{1 / \alpha}-1\right) \mathrm{d} \xi_{1} \mathrm{~d} \xi_{2} \mathrm{~d} \xi_{3}, \\
\left.\frac{\partial I}{\partial y}\right|_{y=1}= & \int_{\Delta} S\left(\xi_{1}, \xi_{2}, \xi_{3}\right)\left(1+\xi_{3}^{\gamma}-\xi_{1}^{\gamma}-\xi_{2}^{\gamma}\right) \delta\left(1+\xi_{3}-\xi_{1}-\xi_{2}\right) \\
& \times\left[\xi_{1} \ln \left(\frac{1}{\xi_{1}}\right)+\xi_{2} \ln \left(\frac{1}{\xi_{2}}\right)-\xi_{3} \ln \left(\frac{1}{\xi_{3}}\right)\right] \delta\left(\xi_{1}^{1 / \alpha}+\xi_{2}^{1 / \alpha}+\xi_{3}^{1 / \alpha}-1\right) \mathrm{d} \xi_{1} \mathrm{~d} \xi_{2} \mathrm{~d} \xi_{3}
\end{aligned}
$$

with

$$
S\left(\xi_{1}, \xi_{2}, \xi_{3}\right)=\frac{4 \pi}{\alpha^{4}}\left(\xi_{1} \xi_{2} \xi_{3}\right)^{((\beta / 2+1) / \alpha)-1-\gamma} .
$$

The sign of each integral above is determined by the factor (see [15])

$$
f(\gamma)=1+\xi_{3}^{\gamma}-\xi_{1}^{\gamma}-\xi_{2}^{\gamma}
$$

It is found that $f(\gamma)$ is positive when

$$
\gamma<0 \quad \text { or } \quad \gamma>1
$$


Table 1

Signs of the fluxes for the Kolmogorov-type solutions, $\alpha=\frac{1}{2}$

\begin{tabular}{lllllll}
\hline$\beta$ & -1 & $-\frac{3}{4}$ & $-\frac{1}{2}$ & $-\frac{1}{4}$ & 0 & +3 \\
$\gamma_{Q}$ & $\frac{1}{3}$ & $\frac{2}{3}$ & 1 & $\frac{4}{3}$ & $\frac{5}{3}$ & $\frac{17}{3}$ \\
Sign of $Q_{0}$ & + & + & 0 & $\frac{5}{3}$ & $\frac{2}{6}$ & - \\
$\gamma_{P}$ & $\frac{2}{3}$ & 1 & $\frac{4}{3}$ & + & + & + \\
Sign of $P_{0}$ & - & 0 & + & + & & + \\
\hline
\end{tabular}

For the same values of $\beta$ as those considered by MMT and the additional value $\beta=+3$, Table 1 displays the corresponding frequency slopes from Eqs. (4.7) and (4.8) and the signs of $Q_{0}, P_{0}$ according to the criterion (5.5).

Our calculations show that WT theory should work most successfully for $\beta=0$ (instead of $\beta=-1$ in [7]) at which they yield both $Q_{0}<0$ and $P_{0}>0$. Incidentally, MMT reported the smallest difference between numerics and theory for $\beta=0$. The cases with spectral slopes less steep than the Rayleigh-Jeans distribution (i.e. $\gamma<1$ ) are non-physical. At best, a thermodynamic equilibrium is expected in the conservative regime. Hence, we cannot strictly rely on the Kolmogorov-type exponents for $\beta=-1,-\frac{3}{4}$ to compare with the numerical results in forced regimes. Note that for $\beta=-\frac{1}{2}$, although we find $P_{0}>0$, a pure thermodynamic equilibrium state (i.e. $\gamma=1$ ) is predicted instead of the inverse cascade. This is however not valid because of the necessity for a finite flux of particles towards $\omega=0$. The direct cascade may then be influenced one way or another, possibly making the theory not applicable to the whole spectrum. Using both criteria (5.5), we deduce that the fluxes of particles and energy simultaneously have the correct signs in the region of parameter

$$
\beta<-\frac{3}{2} \text { and } \beta>2 \alpha-\frac{3}{2},
$$

or

$$
\beta<-\frac{3}{2} \quad \text { and } \beta>-\frac{1}{2} \quad \text { if } \alpha=\frac{1}{2} .
$$

Since the strength of nonlinearity decreases with $\beta$, the case $\beta<-\frac{3}{2}$, which is close to a linear problem, is not interesting from a general viewpoint and may raise some difficulties in numerical studies.

Restricting again to $\alpha=\frac{1}{2}$ and $\beta=0$, one has for the spectrum

$$
n(\omega)=a P^{1 / 3} \omega^{-2},
$$

where $P$ is the flux of energy towards high frequencies and

$$
a=\left(\left.\frac{\partial I}{\partial y}\right|_{y=1}\right)^{-1 / 3}
$$

is the Kolmogorov constant. Numerical calculations give for $a$

$$
a=0.376 .
$$

An important question is the stability of the stationary spectra. This question was studied by Balk and Zakharov in [17] from a general point of view. The particular situation discussed in the present paper requires an additional study based on the work [17]. However, one should note that instability of the present spectra is unlikely. The reason is that the stationary spectra are solutions of the kinetic equation, which is not sensitive to changing the sign of the nonlinearity in the dynamical equation. In other words, if the Kolmogorov solution was unstable, it would be unstable in both cases. Since, we observe the Kolmogorov spectrum in the numerical simulation for one of the signs of nonlinearity, instability is unlikely. 


\section{Solitons and quasisolitons}

Besides random radiative waves, solitons are the most interesting features of nonlinear Hamiltonian models such as the focusing NLS. These localized coherent structures can naturally emerge and persist as the result of the stable competition between nonlinear and dispersive mechanisms. It is known that they act as statistical attractors to which the system relaxes and they can influence the dynamics in a substantial way.

Equally important coherent structures are quasisolitons. They could be defined as solitons having finite but long enough life time. Solitons and quasisolitons can be compared with stable and unstable elementary particles. Formally, both solitons and quasisolitons are defined as solutions of Eq. (2.3) of the form

$$
\hat{\psi}_{k}(t)=\mathrm{e}^{\mathrm{i}(\Omega-k V) t} \hat{\phi}_{k} .
$$

Here $\Omega$ and $V$ are constants. In the $x$-space,

$$
\psi(x, t)=\mathrm{e}^{\mathrm{i} \Omega t} \xi(x-V t),
$$

where $\xi(x)$ is the inverse Fourier transform of $\hat{\phi}_{k}$ and $V$ is the soliton velocity. The amplitude $\hat{\phi}_{k}$ satisfies the integral equation

$$
\hat{\phi}_{k}=-\frac{1}{\Omega-k V+\omega(k)} \int T_{123 k} \hat{\phi}_{1} \hat{\phi}_{2} \hat{\phi}_{3}^{*} \delta\left(k_{1}+k_{2}-k_{3}-k\right) \mathrm{d} k_{1} \mathrm{~d} k_{2} \mathrm{~d} k_{3} .
$$

The "classical" or "true" soliton is a localized solution of Eq. (6.3). In this case,

$$
|\xi(x)|^{2} \rightarrow 0 \quad \text { as }|x| \rightarrow \infty .
$$

This implies that $\hat{\phi}_{k}$ is a continuous function which has no singularities for real $k$. Thus the denominator in Eq. (6.3) should not vanish on the real axis

$$
\Omega-k V+\omega(k) \neq 0, \quad-\infty<k<+\infty .
$$

For $\omega(k)=|k|^{\alpha}$ and $\alpha<1$, the last condition is violated for any $V \neq 0$. So "true solitons" can exist only if $V=0$.

Next, we show that "true" solitons can only exist for $\lambda=-1$. Eq. (6.3) can be rewritten in the variational form

$$
\delta(H+\Omega N)=0 .
$$

Obviously, $\Omega>0$ should hold (otherwise, the denominator (6.5) has zeroes). Since

$$
T_{123 k}=\lambda\left|k_{1} k_{2} k_{3} k\right|^{\beta / 4}, \quad \lambda= \pm 1,
$$

the Hamiltonian is positive for $\lambda=+1$ and Eq. (6.6) can be satisfied only if $\hat{\phi}_{k} \equiv 0$. There are no solitons in this case. Meanwhile, solitons can exist for $\lambda=-1$. A rigorous proof of existence is beyond the frame of this paper.

Quasisolitons are a more sophisticated object. Let us allow the denominator (6.5) to have a zero at $k=k_{0}$ and suppose that $\hat{\phi}_{k}$ is a function which is sharply localized near the wave number $k=k_{m}$. Let the width of $\hat{\phi}_{k}$ near $k=k_{m}$ be $\kappa$. One can introduce

$$
T(k)=\int T_{123 k} \hat{\phi}_{1} \hat{\phi}_{2} \hat{\phi}_{3}^{*} \delta\left(k_{1}+k_{2}-k_{3}-k\right) \mathrm{d} k_{1} \mathrm{~d} k_{2} \mathrm{~d} k_{3} .
$$

We might expect that

$$
T\left(k_{0}\right) \simeq \mathrm{e}^{-C\left(\left|k_{0}-k_{m}\right| / \kappa\right)}\left|\hat{\phi}\left(k_{m}\right)\right|^{2} \hat{\phi}\left(k_{m}\right) .
$$


In other words, $\hat{\phi}_{k}$ has a pole at $k=k_{0}$ but the residue at this pole is exponentially small. It means that the soliton (6.2) is not exactly localized and goes to a very small-amplitude monochromatic wave with wave number $k=k_{0}$ as $x \rightarrow-\infty$.

If one eliminates the pole from $\hat{\phi}_{k}$, one gets a quasisoliton, which is a stationary solution of (2.3) only approximately. Such a quasisoliton lives for a finite time. If this time is long enough, the quasisoliton could become the basic unit of wave turbulence. This is what we believe may happen in the MMT model with positive nonlinearity.

\section{Soliton stability and collapse}

Coherent structures can play a role in wave turbulence only if they are stable. For $\lambda=-1$, a soliton satisfies the equation

$$
\left(\Omega+|k|^{\alpha}\right) \hat{\phi}_{k}=\int\left|k_{1} k_{2} k_{3} k\right|^{\beta / 4} \hat{\phi}_{1} \hat{\phi}_{2} \hat{\phi}_{3}^{*} \delta\left(k_{1}+k_{2}-k_{3}-k\right) \mathrm{d} k_{1} \mathrm{~d} k_{2} \mathrm{~d} k_{3} .
$$

The free parameter $\Omega$ can be eliminated by the scaling

$$
\hat{\phi}_{k}=\Omega^{-(\beta-\alpha+2) / 2 \alpha} \chi\left(\Omega^{-1 / \alpha} k\right),
$$

where $\chi(\xi)$ satisfies the equation

$$
\left(1+|\xi|^{\alpha}\right) \chi(\xi)=\int\left|\xi_{1} \xi_{2} \xi_{3} \xi\right|^{\beta / 4} \chi_{1} \chi_{2} \chi_{3}^{*} \delta\left(\xi_{1}+\xi_{2}-\xi_{3}-\xi\right) \mathrm{d} \xi_{1} \mathrm{~d} \xi_{2} \mathrm{~d} \xi_{3} .
$$

Let us calculate the total wave action in the soliton

$$
N=\int\left|\hat{\phi}_{k}\right|^{2} \mathrm{~d} k=\Omega^{-(\beta-\alpha+1) / \alpha} N_{0},
$$

where

$$
N_{0}=\int|\chi|^{2} \mathrm{~d} \xi
$$

The stability question can be answered by computing $\partial N / \partial \Omega$. As is well-known (see [18]), a soliton is stable if $\partial N / \partial \Omega>0$. In our case,

$$
\frac{\partial N}{\partial \Omega}=-\left(\frac{\beta-\alpha+1}{\alpha}\right) \frac{N}{\Omega} .
$$

The soliton is stable if

$$
\beta<\alpha-1,
$$

otherwise the soliton is unstable. For $\alpha=\frac{1}{2}$, the condition of soliton instability reads

$$
\beta>-\frac{1}{2} \text {. }
$$

This condition is satisfied in all the cases we studied.

The soliton instability leads us to guess that the typical coherent structure in the case of negative nonlinearity is a collapsing singularity. Typically, the formation of such singularities is described by self-similar solutions of the initial equations. Eq. (2.3) has the following family of self-similar solutions

$$
\hat{\psi}(k, t)=\left(t_{0}-t\right)^{p+\mathrm{i} \epsilon} \chi\left[k\left(t_{0}-t\right)^{1 / \alpha}\right],
$$


where $p=(\beta-\alpha+2) / 2 \alpha$ and $\epsilon$ is an arbitrary constant. $\chi(\xi)$ satisfies the equation

$$
\mathrm{i}(p+\mathrm{i} \epsilon) \chi+\frac{\mathrm{i}}{\alpha} \xi \chi^{\prime}+|\xi|^{\alpha} \chi+\lambda \int\left|\xi_{1} \xi_{2} \xi_{3} \xi\right|^{\beta / 4} \chi_{1} \chi_{2} \chi_{3}^{*} \delta\left(\xi_{1}+\xi_{2}-\xi_{3}-\xi\right) \mathrm{d} \xi_{1} \mathrm{~d} \xi_{2} \mathrm{~d} \xi_{3}=0
$$

The soliton (7.9) should stay finite when $t \rightarrow t_{0}$. This requirement imposes the following asymptotic behavior on $\chi(\xi)$

$$
\chi(\xi) \rightarrow C \xi^{(-\beta+\alpha-2) / 2}, \quad \xi \rightarrow 0 .
$$

At time $t=t_{0}$, Eq. (7.9) turns to the powerlike function

$$
\hat{\psi}_{k} \rightarrow C k^{-v}, \quad v=\frac{1}{2}(\beta-\alpha+2) .
$$

In reality, the self-similar solution is realized in $x$-space in a finite domain of order $L$. Hence, the solution (7.12) should be cut off at $k \simeq 1 / L$. In $k$-space, Eq. (7.9) represents the formation of a powerlike "tail" (7.12). The wave action concentrated in this tail must be finite. Therefore the integral

$$
\int_{0}^{\infty}\left|\hat{\psi}_{k}\right|^{2} \mathrm{~d} k
$$

should converge as $k \rightarrow \infty$. It leads to the condition on parameters

$$
\beta>\alpha-1,
$$

which coincides with the condition for soliton instability.

Let us plug (7.9) into the Hamiltonian in Fourier space

$$
\begin{aligned}
H & =\int \omega(k)\left|\hat{\psi}_{k}\right|^{2} \mathrm{~d} k+\int T_{123 k} \hat{\psi}_{1} \hat{\psi}_{2} \hat{\psi}_{3}^{*} \hat{\psi}_{k}^{*} \delta\left(k_{1}+k_{2}-k_{3}-k\right) \mathrm{d} k_{1} \mathrm{~d} k_{2} \mathrm{~d} k_{3} \mathrm{~d} k \\
& =\left(t_{0}-t\right)^{(\beta-2 \alpha+1) / \alpha} H_{0},
\end{aligned}
$$

where

$$
H_{0}=\int|\xi|^{\alpha}|\chi|^{2} \mathrm{~d} \xi+\lambda \int\left|\xi_{1} \xi_{2} \xi_{3} \xi\right|^{\beta / 4} \chi_{1} \chi_{2} \chi_{3}^{*} \chi^{*} \delta\left(\xi_{1}+\xi_{2}-\xi_{3}-\xi\right) \mathrm{d} \xi_{1} \mathrm{~d} \xi_{2} \mathrm{~d} \xi_{3} \mathrm{~d} \xi .
$$

If $\alpha-1<\beta<2 \alpha-1$, then $H \rightarrow \infty$ as $t \rightarrow t_{0}$, unless

$$
H_{0}=0 .
$$

Apparently, this condition can be satisfied only for $\lambda=-1$ (negative nonlinearity). The condition (7.16) imposes implicitly a constraint on the constant $\epsilon$. In fact, it can be realized only at one specific value of $\epsilon$, which is an eigenvalue of the boundary problem (7.10) with the boundary conditions

$$
\chi(\xi) \rightarrow C \xi^{(-\beta+\alpha-2) / 2}, \quad \xi \rightarrow 0, \quad|\chi(\xi)| \rightarrow \infty, \quad|\xi| \rightarrow \infty .
$$

In the case $\beta>2 \alpha-1, H \rightarrow 0$ as $t \rightarrow t_{0}$. There is no limitation on the value of $H_{0}$ and the singularity can take place for either sign of $\lambda$. If $v<1$ in Eq. (7.12) or $\alpha-1<\beta<\alpha$, a collapse is the formation of an integrable singularity in $x$-space. If $\nu>1$ or $\beta>\alpha$, the singularity is the formation of a discontinuity of the function $\psi(x)$ or its derivatives.

The formation of singularities leads to the formation in $k$-space of a powerlike spectrum

$$
n_{k} \simeq\left|\hat{\psi}_{k}\right|^{2} \simeq|k|^{-2 v} \simeq|k|^{-\beta+\alpha-2} .
$$


For $\alpha=\frac{1}{2}$ and $\beta=0$, one obtains

$$
n_{k} \simeq|k|^{-3 / 2} \simeq \omega^{-3} .
$$

This spectrum can be called Phillips spectrum by analogy to the well-known " $\omega^{-5}$ spectrum" for deep water waves [19]. As $\omega \rightarrow \infty$, it decays faster than Kolmogorov spectra.

\section{More on quasisolitons}

Let us consider again the case of negative nonlinearity $\lambda=-1$ and denote

$$
F=-\Omega+k V-\omega(k)=-\Omega+k V-|k|^{\alpha} .
$$

If $V=0$ and $\Omega>0,|F|$ has a minimum at $k=0$. The Fourier transform of the solution $\hat{\phi}_{k}$ is concentrated near this minimum in a domain of width

$$
\kappa \simeq \Omega^{1 / \alpha} .
$$

Assuming that the soliton is smooth in $x$-space, $\hat{\phi}_{k}$ decays very fast outside of the domain (8.2). So far we assumed that $V=0$. Let now $V$ be positive but very small. Then the denominator $F$ has a zero at $k=k_{0} \simeq V^{1 /(\alpha-1)}$. For small $V$, the wave number $k_{0}$ is much larger than $\kappa$ and this zero occurs very far from the domain which supports the soliton. This means that $\hat{\phi}_{k}$ has a pole at $k=k_{0}$, but the residue at this pole is very small. The presence of this pole means that the stationary solution (6.3) looks in the $x$-space like a soliton, which is not completely localized. As $x \rightarrow+\infty$, it becomes a monochromatic wave with wave number $k_{0}$ and negligibly small amplitude.

If this "wave tail" is cut off in the initial data, one has a "quasisoliton" which slowly decays due to radiation of energy in the right direction. If $V$ is small enough, the lifetime of the quasisoliton is very long and its shape is close to the shape of "real" solitons.

It is unlikely that quasisolitons play an important role in wave turbulence at negative nonlinearity. If $V$ is not small, their lifetime is too short; if $V$ is small, they are unstable like real solitons. Quasisolitons are more relevant in the case of positive nonlinearity $\lambda=+1$.

Let us choose an arbitrary $k=k_{m}>0$ and plug in Eq. (6.3)

$$
V=\alpha k_{m}^{\alpha-1}, \quad \Omega=-(1-\alpha) k_{m}^{\alpha}-\frac{1}{2} \alpha(1-\alpha) k_{m}^{\alpha-2} q^{2} .
$$

Then

$$
F=k_{m}^{\alpha}-|k|^{\alpha}+\alpha k_{m}^{\alpha-1}\left(k-k_{m}\right)+\frac{1}{2} \alpha(1-\alpha) k_{m}^{\alpha-2} q^{2} .
$$

Note that if $\alpha<1$ then $F$ has a zero at $k=k_{0}<0$ for any $k_{m}$. Hence, $1 / F$ always has a pole on the negative real axis, and the soliton (6.3) cannot be a real soliton. But if $q^{2} \ll k_{m}^{2}, 1 / F$ has a sharp maximum at $k \simeq k_{m}$. Introducing

$$
\kappa=\left|k-k_{m}\right|,
$$

one has approximately

$$
F \simeq \frac{1}{2} \alpha(1-\alpha) k_{m}^{\alpha-2}\left[\kappa^{2}+q^{2}\right]
$$

and one gets for the width of the maximum of $1 / F$

$$
\kappa \simeq q .
$$


If $\kappa \ll\left|k_{0}\right|$, one can construct a quasisoliton which is supported in $k$-space near $k_{m}$. In the general case, $\left|k_{0}\right| \simeq k_{m}$. If $\alpha=\frac{1}{2}$ and $q=0$, one can easily find

$$
k_{0}=-(\sqrt{2}-1)^{2} k_{m} .
$$

The quasisoliton moves to the right direction with the velocity $V\left(k_{m}\right)$ and radiates backward monochromatic waves of wave number $k_{0}$. The shape of the quasisoliton can be found explicitly in the limit $q \rightarrow 0$. Now $\kappa \ll k_{m}$ and one has approximately

$$
\int\left|k_{1} k_{2} k_{3} k\right|^{\beta / 4} \hat{\phi}_{1} \hat{\phi}_{2} \hat{\phi}_{3}^{*} \delta\left(k_{1}+k_{2}-k_{3}-k\right) \mathrm{d} k_{1} \mathrm{~d} k_{2} \mathrm{~d} k_{3} \simeq k_{m}^{\beta} \int \hat{\phi}_{1} \hat{\phi}_{2} \hat{\phi}_{3}^{*} \delta\left(\kappa_{1}+\kappa_{2}-\kappa_{3}-\kappa\right) \mathrm{d} \kappa_{1} \mathrm{~d} \kappa_{2} \mathrm{~d} \kappa_{3} .
$$

Taking into account Eq. (8.6), one can rewrite Eq. (6.3) as

$$
\frac{1}{2} \alpha(1-\alpha) k_{m}^{\alpha-2}\left(\kappa^{2}+q^{2}\right) \hat{\phi}_{\kappa}=k_{m}^{\beta} \int \hat{\phi}_{1} \hat{\phi}_{2} \hat{\phi}_{3}^{*} \delta\left(\kappa_{1}+\kappa_{2}-\kappa_{3}-\kappa\right) \mathrm{d} \kappa_{1} \mathrm{~d} \kappa_{2} \mathrm{~d} \kappa_{3} .
$$

With the help of inverse Fourier transform, one can transform (8.10) into the stationary NLS

$$
\frac{1}{2} \alpha(1-\alpha) k_{m}^{\alpha-2}\left[-\frac{\partial^{2} \phi}{\partial x^{2}}+q^{2} \phi\right]=k_{m}^{\beta}|\phi|^{2} \phi
$$

which has the soliton solution

$$
\phi(x)=\sqrt{\frac{\alpha(1-\alpha)}{k_{m}^{\beta-\alpha+2}}} \frac{q}{\cosh q x} .
$$

It gives the following approximate quasisoliton solution of Eq. (2.1) with $\lambda=+1$ :

$$
\psi(x, t)=\phi(x-V t) \mathrm{e}^{\mathrm{i} \Omega t+\mathrm{i} k_{m}(x-V t)}, \quad \Omega=-(1-\alpha) k_{m}^{\alpha}-\frac{1}{2} \alpha(1-\alpha) k_{m}^{\alpha-2} q^{2}, \quad V=\alpha k_{m}^{\alpha-1} .
$$

The quasisoliton (8.13) is an "envelope soliton", which can be obtained directly from Eq. (2.1). Simply inject

$$
\psi(x, t)=U(x, t) \mathrm{e}^{-\mathrm{i}(1-\alpha) k_{m}^{\alpha} t+\mathrm{i} k_{m}(x-V t)},
$$

and use the binomial expansion

$$
\left|\frac{\partial}{\partial x}\right|^{a} \mathrm{e}^{\mathrm{i} k x} U=\mathrm{e}^{\mathrm{i} k x}\left[|k|^{a} U+a|k|^{a-1}\left(-\mathrm{i} \frac{\partial}{\partial x}\right) U+\frac{1}{2} a(a-1)|k|^{a-2}\left(-\mathrm{i} \frac{\partial}{\partial x}\right)^{2} U+\cdots\right] .
$$

Plugging Eq. (8.15) into Eq. (2.1) with $\lambda=+1$, one obtains a differential equation of infinite order

$$
\mathrm{i}\left(\frac{\partial U}{\partial t}+V \frac{\partial U}{\partial x}\right)=L_{2} U+L_{3} U+\cdots, \quad V=\alpha k_{m}^{\alpha-1} .
$$

Here

$$
\begin{aligned}
& L_{2} U=\frac{1}{2} \alpha(1-\alpha) k_{m}^{\alpha-2} \frac{\partial^{2} U}{\partial x^{2}}+k_{m}^{\beta}|U|^{2} U \\
& L_{3} U=\mathrm{i}\left[\frac{1}{6} \alpha(\alpha-1)(\alpha-2) k_{m}^{\alpha-3} \frac{\partial^{3} U}{\partial x^{3}}-\beta k_{m}^{\beta-1}|U|^{2} \frac{\partial U}{\partial x}\right] .
\end{aligned}
$$


Taking into consideration only the first non-trivial term $L_{2} U$, one gets the non-stationary NLS

$$
\mathrm{i}\left(\frac{\partial U}{\partial t}+V \frac{\partial U}{\partial x}\right)=\frac{1}{2} \alpha(1-\alpha) k_{m}^{\alpha-2} \frac{\partial^{2} U}{\partial x^{2}}+k_{m}^{\beta}|U|^{2} U .
$$

It has a soliton solution

$$
U(x, t)=\phi(x-V t) \mathrm{e}^{-(1 / 2) \mathrm{i} \alpha(1-\alpha) k_{m}^{\alpha-2} q^{2} t} .
$$

To find the shape of the quasisoliton more accurately, one should keep in the right-hand side of Eq. (8.15) a finite (but necessary odd!) number of terms. The expansion in Eq. (8.16) runs in powers of the parameter $q / k_{m}$. Note that one cannot find the lifetime of the quasisoliton. The lifetime grows as $\mathrm{e}^{\left|k_{0}\right| / q}$ and its calculation is beyond the perturbation expansion.

As a matter of fact, the parameter

$$
\epsilon=\frac{q}{k_{m}}
$$

is crucial for quasisolitons. The smaller it is, the closer the quasisoliton is to a "real soliton". The amplitude of a quasisoliton is proportional to $\epsilon$. Quasisolitons of small amplitude satisfy the integrable NLS and are stable. It is not obvious for quasisolitons of finite amplitude. One can guess that at least in the case $\beta>0$, when collapse is not forbidden, there is a critical value of the amplitude of a quasisoliton $\epsilon_{\mathrm{c}}$ such that for $\epsilon>\epsilon_{\mathrm{c}}$ it is unstable and generates a singularity at a finite time. Our numerical experiments confirm this conjecture for $\beta=+3$.

Quasisolitons move with different velocities and collide. If the amplitudes of the quasisolitons are small and their velocities are close, they obey the NLS and their interaction is elastic. One can guess that the same holds for small-amplitude quasisolitons even if their velocities are quite different. This is not obvious for quasisolitons of moderate amplitude. One can think that their interaction is inelastic and leads to the merging and formation of a quasisoliton of larger amplitude.

\section{Nonlinear frequency shift}

Let us consider one more important nonlinear effect. In a linear system, the harmonic of wave number $k$ oscillates with the frequency $\omega(k)$. In the presence of nonlinearity, the frequency changes due to the interaction with other harmonics. In a weakly nonlinear system, the frequency is modified by a functional depending linearly on the spectrum

$$
\omega(k) \rightarrow \omega(k)+\int T_{1 k} n_{1} \mathrm{~d} k_{1} .
$$

It is easy to show that $T_{1 k}$ can be expressed in terms of the coefficient $T_{123 k}$ in Eq. (2.3) as

$$
T_{1 k}=2 T_{1 k 1 k} .
$$

For the MMT model,

$$
T_{1 k}=2 \lambda\left(k_{1} k\right)^{\beta / 2} \text {. }
$$

For $\beta=0$,

$$
T_{1 k}=2 \lambda= \pm 2,
$$


and

$$
\omega^{ \pm}(k)=\omega(k) \pm 2 N,
$$

where $N=\int\left|\hat{\psi}_{k}\right|^{2} \mathrm{~d} k$ is the total number of particles.

In the general case $\beta \neq 0$, renormalization of the frequency leads to modified resonance conditions (2.8) and (2.9). But in the particular case $\beta=0$, renormalization terms in Eq. (2.9) cancel and the resonance conditions in the first nonlinear approximation remain unchanged. At the same time, the difference of frequencies for different signs of nonlinearity has the form

$$
\omega^{+}(k)-\omega^{-}(k)=4 N .
$$

In our case, it does not depend on the wave number.

\section{On the MMT model spectrum}

In [7], MMT found that in the case of positive nonlinearity the spectrum of wave turbulence is well described by the formula (MMT spectrum)

$$
n_{k} \simeq|k|^{-1-(\beta+\alpha) / 2} .
$$

They checked this result for $\alpha=\frac{1}{2}$ and different values of $\beta$. Our experiments are in agreement with (10.1). In [8-10], it was found that the MMT spectrum can appear for either sign of nonlinearity. So far there is no proper theoretical derivation of the MMT spectrum. In this section, we offer some heuristic derivation of (10.1).

Assuming formula (3.2) to be exact, the problem of closure for the equation on particle number lies in the expression of Im $J_{123 k}$ in terms of $n_{k}$. This expression should a priori satisfy the conditions of symmetry

$$
\operatorname{Im} J_{123 k}=\operatorname{Im} J_{213 k}=\operatorname{Im} J_{12 k 3}=-\operatorname{Im} J_{3 k 12} .
$$

Moreover, one can assume that the nonlinearity is weak and that the wave energy is roughly

$$
E \simeq \int \omega(k) n_{k} \mathrm{~d} k
$$

From conservation of energy, one obtains

$$
\int T_{123 k}\left(\omega_{1}+\omega_{2}-\omega_{3}-\omega\right) \operatorname{Im} J_{123 k} \mathrm{~d} k_{1} \mathrm{~d} k_{2} \mathrm{~d} k_{3} \mathrm{~d} k=0
$$

Hence, one must have

$$
\operatorname{Im} J_{123 k} \simeq \delta\left(\omega_{1}+\omega_{2}-\omega_{3}-\omega\right) .
$$

For Gaussian wave turbulence, the real part of $J_{123 k}$ is given by Eq. (3.3) and dimensional analysis gives

$$
\operatorname{Re} J_{123 k} \simeq \frac{n_{k}^{2}}{k}
$$

Up to this point, our consideration was more or less rigorous. Now, we present a heuristic conjecture. We suppose that the imaginary part of the four-wave correlator has the same scaling as the real part. In other words, it is quadratic in $n_{k}$. 
If one takes into account the necessary conditions (10.2) and (10.4) and the scaling (10.6) for $\operatorname{Im} J_{123 k}$, there are only a few possibilities for the construction of $\operatorname{Im} J_{123 k}$. We offer the following closure:

$$
\operatorname{Im} J_{123 k}=a\left(\frac{\partial \omega_{1}}{\partial k_{1}}+\frac{\partial \omega_{2}}{\partial k_{2}}+\frac{\partial \omega_{3}}{\partial k_{3}}+\frac{\partial \omega}{\partial k}\right) \delta\left(\omega_{1}+\omega_{2}-\omega_{3}-\omega\right)\left(n_{1} n_{2}-n_{3} n_{k}\right),
$$

where $a \ll 1$ is a dimensionless constant. The closure leads to the kinetic equation

$$
\begin{aligned}
\frac{\partial n_{k}}{\partial t}= & 2 a \int T_{123 k}\left(n_{1} n_{2}-n_{3} n_{k}\right)\left(\frac{\partial \omega_{1}}{\partial k_{1}}+\frac{\partial \omega_{2}}{\partial k_{2}}+\frac{\partial \omega_{3}}{\partial k_{3}}+\frac{\partial \omega}{\partial k}\right) \delta\left(\omega_{1}+\omega_{2}-\omega_{3}-\omega\right) \\
& \times \delta\left(k_{1}+k_{2}-k_{3}-k\right) \mathrm{d} k_{1} \mathrm{~d} k_{2} \mathrm{~d} k_{3} .
\end{aligned}
$$

It is easy to check that the Kolmogorov solution of Eq. (10.8) leads to the MMT spectrum. Eq. (10.8) resembles the Boltzmann's equation for interacting particles. Apparently, it can make sense only if $a T_{123 k}>0$. Otherwise, the $\mathcal{H}$-theorem and the second law of thermodynamics will be violated. We must stress that the formula (10.7) is heuristic and has no rigorous justification.

\section{Particle and energy balance}

In the presence of damping and linear instability, Eq. (2.3) can be written in the form

$$
\mathrm{i} \frac{\partial \hat{\psi}_{k}}{\partial t}=\frac{\delta H}{\delta \hat{\psi}_{k}^{*}}+\mathrm{i} D(k) \hat{\psi}_{k}
$$

where

$$
H=\int \omega(k)\left|\hat{\psi}_{k}\right|^{2} \mathrm{~d} k+\frac{1}{2} \int T_{123 k} \hat{\psi}_{1} \hat{\psi}_{2} \hat{\psi}_{3}^{*} \hat{\psi}_{k}^{*} \delta\left(k_{1}+k_{2}-k_{3}-k\right) \mathrm{d} k_{1} \mathrm{~d} k_{2} \mathrm{~d} k_{3} \mathrm{~d} k,
$$

is the Hamiltonian, $D(k)$ is the damping or the growth rate of instability depending on its sign.

Let $N=\int\left|\hat{\psi}_{k}\right|^{2} \mathrm{~d} k$ be the total number of particles in the system. From (11.1), one can obtain the exact equation for the particle balance

$$
\frac{\mathrm{d} N}{\mathrm{~d} t}=Q=2 \int D(k)\left|\hat{\psi}_{k}\right|^{2} \mathrm{~d} k .
$$

After averaging, one has

$$
\frac{\mathrm{d}\langle N\rangle}{\mathrm{d} t}=2 \int D(k) n_{k} \mathrm{~d} k=\langle Q\rangle .
$$

The total mean flux of particles $\langle Q\rangle$ is a linear functional of $n_{k}$ at any level of nonlinearity.

For the total flux of energy, one has the exact identity

$$
\begin{aligned}
P=\frac{\mathrm{d} H}{\mathrm{~d} t}= & 2 \int \omega(k) D(k)\left|\hat{\psi}_{k}\right|^{2} \mathrm{~d} k+\frac{1}{2} \int\left[D\left(k_{1}\right)+D\left(k_{2}\right)+D\left(k_{3}\right)+D(k)\right] T_{123 k} \hat{\psi}_{1} \hat{\psi}_{2} \hat{\psi}_{3}^{*} \hat{\psi}_{k}^{*} \\
& \times \delta\left(k_{1}+k_{2}-k_{3}-k\right) \mathrm{d} k_{1} \mathrm{~d} k_{2} \mathrm{~d} k_{3} \mathrm{~d} k .
\end{aligned}
$$

For the averaged density of energy, one has

$$
\begin{aligned}
\langle P\rangle= & 2 \int \omega(k) D(k) n_{k} \mathrm{~d} k+\frac{1}{2} \int\left[D\left(k_{1}\right)+D\left(k_{2}\right)+D\left(k_{3}\right)+D(k)\right] T_{123 k} \operatorname{Re} J_{123 k} \\
& \times \delta\left(k_{1}+k_{2}-k_{3}-k\right) \mathrm{d} k_{1} \mathrm{~d} k_{2} \mathrm{~d} k_{3} \mathrm{~d} k .
\end{aligned}
$$


Assuming that Gaussian statistics holds, one can write

$$
\operatorname{Re} J_{123 k} \simeq n_{1} n_{2}\left[\delta\left(k_{1}-k_{3}\right)+\delta\left(k_{1}-k\right)\right],
$$

and one obtains after simple calculations

$$
\langle P\rangle=2 \int \tilde{\omega}(k) D(k) n_{k} \mathrm{~d} k,
$$

where $\tilde{\omega}(k)=\omega(k)+\int T_{1 k} n_{1} \mathrm{~d} k_{1}$ is the renormalized frequency.

In the case $\beta=0$ and $T_{1 k}= \pm 2$,

$$
\langle P\rangle=2 \int \omega(k) D(k) n_{k} \mathrm{~d} k+2 \lambda N\langle Q\rangle .
$$

In the stationary state, $\langle Q\rangle=0,\langle P\rangle=0$ and the balance equations are

$$
\begin{aligned}
& \int D(k) n_{k} \mathrm{~d} k=0, \\
& \int \omega(k) D(k) n_{k} \mathrm{~d} k=0 .
\end{aligned}
$$

In this particular case, renormalization of the frequency does not influence the balance equations.

The balance equations (11.10) and (11.11) can be rewritten as

$$
\begin{aligned}
& Q_{0}=Q^{+}+Q^{-}, \\
& P_{0}=P^{+}+P^{-},
\end{aligned}
$$

where $Q_{0}$ and $P_{0}$ are the input of particles and energy in the area of instability $\omega \simeq \omega_{0} . Q^{+}$and $P^{+}$are the sinks of particles and energy in the high frequency region $\omega \sim \omega^{+} . Q^{-}$and $P^{-}$are the sinks in the low frequency region $\omega \sim \omega^{-}$.

Roughly speaking,

$$
\begin{aligned}
& P_{0} \simeq \omega_{0} Q_{0}, \\
& P^{+} \simeq \omega^{+} Q^{+}, \\
& P^{-} \simeq \omega^{-} Q^{-},
\end{aligned}
$$

and the balance equations can be written as

$$
\begin{aligned}
& Q_{0}=Q^{+}+Q^{-}, \\
& \omega_{0} Q_{0} \simeq \omega^{+} Q^{+}+\omega^{-} Q^{-} .
\end{aligned}
$$

Hence

$$
\frac{Q^{+}}{Q^{-}} \simeq \frac{\omega_{0}-\omega^{-}}{\omega^{+}-\omega_{0}}, \quad \frac{P^{+}}{P^{-}} \simeq \frac{\omega^{+}}{\omega^{-}} \frac{\omega_{0}-\omega^{-}}{\omega^{+}-\omega_{0}} .
$$

For $\omega^{-} \sim \omega_{0} \ll \omega^{+}$, one has

$$
\frac{Q^{+}}{Q^{-}} \simeq \frac{\omega_{0}-\omega^{-}}{\omega^{+}}, \quad \frac{P^{+}}{P^{-}} \simeq \frac{\omega_{0}-\omega^{-}}{\omega^{-}} .
$$


In other words, if $\omega_{0} \ll \omega^{+}$, almost all particles are absorbed at low frequencies. The amounts of energy absorbed in both ranges have the same order of magnitude. These conclusions are valid only under the hypothesis of approximate Gaussianity of wave turbulence.

\section{Numerical integration scheme}

The direct method employed to simulate the model is similar to that in [7]. With the aim of observing direct and inverse cascades, the complete equation to be integrated reads

$$
\mathrm{i} \frac{\partial \hat{\psi}_{k}}{\partial t}=\omega(k) \hat{\psi}_{k}+\int T_{123 k} \hat{\psi}_{1} \hat{\psi}_{2} \hat{\psi}_{3}^{*} \delta\left(k_{1}+k_{2}-k_{3}-k\right) \mathrm{d} k_{1} \mathrm{~d} k_{2} \mathrm{~d} k_{3}+\mathrm{i}[F(k)+D(k)] \hat{\psi}_{k}
$$

with

$$
F(k)=\sum_{j} f_{j} \delta\left(k-k_{j}\right) \quad \text { and } \quad D(k)=-v^{-}|k|^{-d^{-}}-v^{+}|k|^{d^{+}} .
$$

The forcing term $F(k)$ denotes an instability localized in a narrow spectral band. The damping part $D(k)$ contains a wave action sink at large scales and an energy sink at small scales. The presence of these two sinks is necessary to reach a stationary regime if two different fluxes are assumed to flow in opposite $k$-directions from the stirred zone. In our experiments, we set $d^{-}=8$ and $d^{+}=16$ unless other values are specified. The purpose of using high-order viscosity (also referred to as hyperviscosity), which separates sharply the inertial and dissipative ranges, is to minimize the effects of dissipation at intermediate scales of the simulated spectrum.

A pseudospectral code solves Eq. (12.1) in a periodic interval of Fourier modes. We define the discrete direct Fourier transform (FT) as

$$
\hat{\psi}\left(k_{n}\right)=\hat{\psi}_{n}=\mathrm{FT}\left(\psi_{j}\right)=\frac{1}{N_{d}} \sum_{j=0}^{N_{d}-1} \psi_{j} \mathrm{e}^{-\mathrm{i} k_{n} x_{j}},
$$

and the discrete inverse Fourier transform $\left(\mathrm{FT}^{-1}\right)$ as

$$
\psi\left(x_{j}\right)=\psi_{j}=\mathrm{FT}^{-1}\left(\hat{\psi}_{n}\right)=\sum_{n=-\left(N_{d} / 2\right)+1}^{N_{d} / 2} \hat{\psi}_{n} \mathrm{e}^{\mathrm{i} k_{n} x_{j}},
$$

where $N_{d}$ is the number of grid points, $k_{n}=2 \pi n / L$ the $n$th wave number, $x_{j}=j L / N_{d}$ the location of the $j$ th grid point and $L$ the size of the computational domain $0<x<L$. We usually choose $L=2 \pi$ so that the $k_{n} \mathrm{~s}$ are integers and the spacing in Fourier space is $\Delta k=1$. In our experiments, quantities defined as integrals along the spectral interval are computed in their discrete forms without any renormalization. For instance, we use for the number of particles the formula

$$
N=\sum_{n=-\left(N_{d} / 2\right)+1}^{N_{d} / 2}\left|\hat{\psi}_{n}\right|^{2},
$$

and for the quadratic part of energy

$$
E=H_{\mathrm{L}}=\sum_{n=-\left(N_{d} / 2\right)+1}^{N_{d} / 2} \omega\left(k_{n}\right)\left|\hat{\psi}_{n}\right|^{2} .
$$


The linear frequency term is treated exactly by an integrating factor technique, removing it from the timestepping procedure. As emphasized by MMT, we thus avoid the natural stiffness of the problem as well as possible numerical instabilities. Consequently, we do not need to shorten the inertial interval by downshifting the cutoff of ultraviolet absorption (as in [5]). The nonlinear term is calculated through the Fast Fourier Transform by first transforming to real space where a multiplication is computed and then transforming back to spectral space. For the multiplication operation, twice the effective number of grid points are required in order to avoid aliasing errors. A fourth-order Runge-Kutta scheme integrates the conservative model in time, giving a solution to which the diagonal factor $\mathrm{e}^{[F(k)+D(k)] \Delta t}$ is applied at each time step $\Delta t$.

\section{Numerical results for $\beta=0, \lambda= \pm 1$}

A series of numerical simulations of Eq. (12.1) with resolution up to $N_{d}=2048$ de-aliased modes has been performed. We choose the case $\beta=0$ as the candidate for testing WT in our experiments. Both cases $\lambda= \pm 1$ are examined, providing an additional test of the theory, and the study is focused on the direct cascade. Forcing is located at large scales and the inertial interval is defined by the right transparency window $k_{\mathrm{f}} \ll k \ll k_{\mathrm{d}}$ (where $k_{\mathrm{f}}$ and $k_{\mathrm{d}}$ are the characteristic wave numbers of forcing and ultraviolet damping, respectively). As displayed in Table 1, the theoretical spectrum which can be realized in this window is

$$
n_{k} \propto k^{-1}
$$

Typically, initial conditions are given by the random noise in the spectral space. Simulations are run until a quasi-steady regime is established which is characterized by small fluctuations of the energy and the number of particles around some mean value. Then time averaging begins and continues for a length of time which significantly exceeds the characteristic time scale of the slowest harmonic from the inertial range (free of the source and the sink). In turn, the time-step of the integration has to provide, at least, accurate enough resolution of the fastest harmonic in the system. As our experiments show, one has to use an even smaller time-step than defined by the last condition: the presence of fast nonlinear events in the system requires the use of a time-step $\Delta t=0.005$, which is 40 times smaller than the smallest linear frequency period. Time averaging with such a small time step leads to a computationally time consuming procedure despite the one-dimensionality of the problem.

From now on, we will present numerical results in the specific situations $v^{-}=196.61(\lambda= \pm 1), v^{+}=$ $5.39 \times 10^{-48}(\lambda=+1)$ or $v^{+}=2.1 \times 10^{-47}(\lambda=-1)$, and $f_{j}=0.2$, non-zero only for $k_{j} \in[6,9](\lambda= \pm 1)$.

The numerical simulations clearly display the development of dynamical chaos and statistically uniform turbulence. Both the amplitude and the phase of each harmonic fluctuate independently of each other. Figs. 1-4 show the behavior of the seventh and eighth harmonics.

Figs. 5-8 show the behavior of the real and imaginary parts of the amplitude of the harmonic $k=200$. One sees amplitude-modulated oscillations with carrying frequency close to the corresponding linear frequency of the harmonic $\omega \simeq 14$.

Figs. 9-12 represent FTs in time of the evolution of the harmonic $k=200$ from the previous pictures. One can see that the maximum of the spectra corresponds to the linear frequency shifted in accordance with the nonlinearity $\operatorname{sign} \lambda= \pm 1$.

Figs. 13 and 14 demonstrate the behavior of the fourth- and sixth-order moments as functions of the second-order moment. They fit the Gaussian laws very well. They provide a justification of the initial conjecture that the statistics of the turbulence is close to Gaussian.

Fig. 15 represents the time evolution of the quadratic energy $E$ for $\lambda= \pm 1$ with the same amplitude of forcing. The curves are plotted over the interval $t \in[5000,10000]$ where the time averaging actually takes place. One 


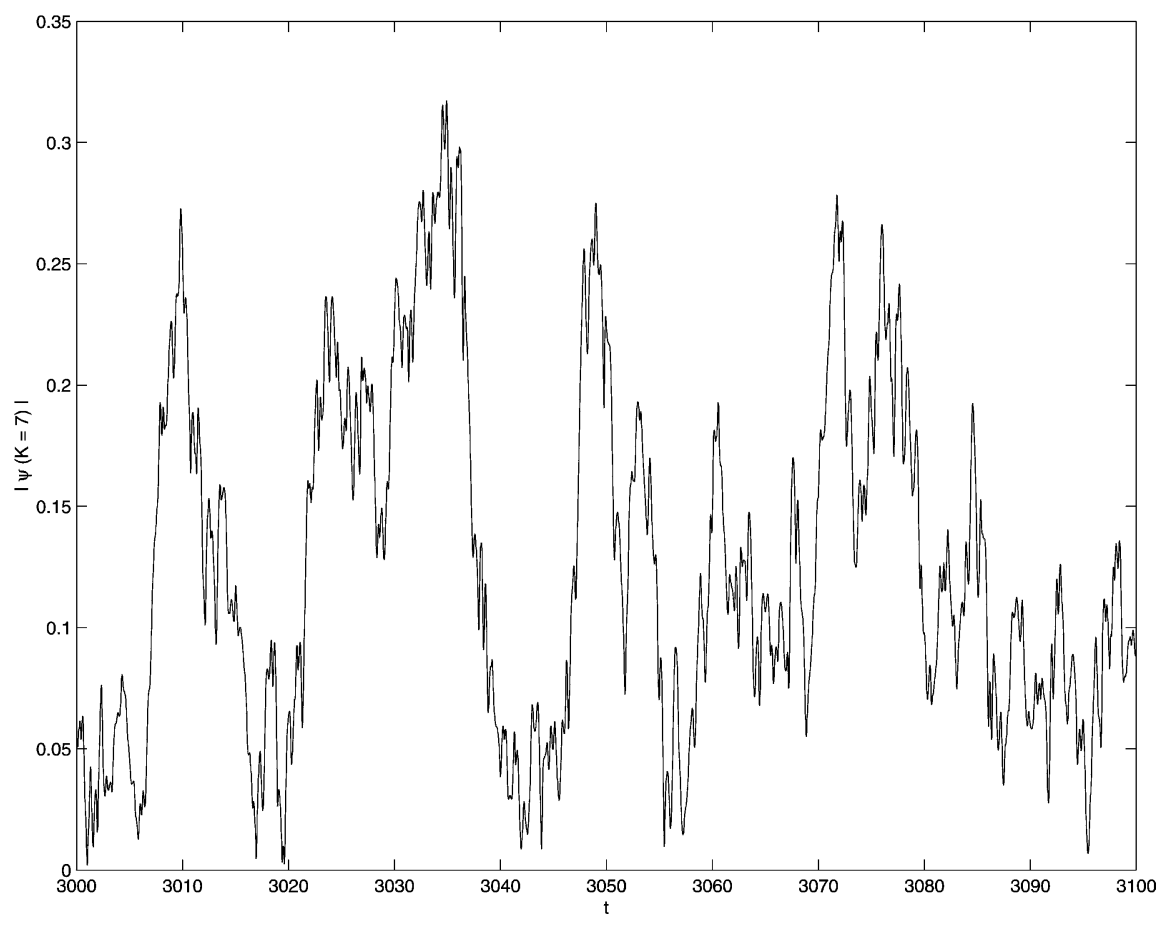

Fig. 1. $\beta=0, \lambda=-1$. Amplitude of the mode $k=7$ vs. time.

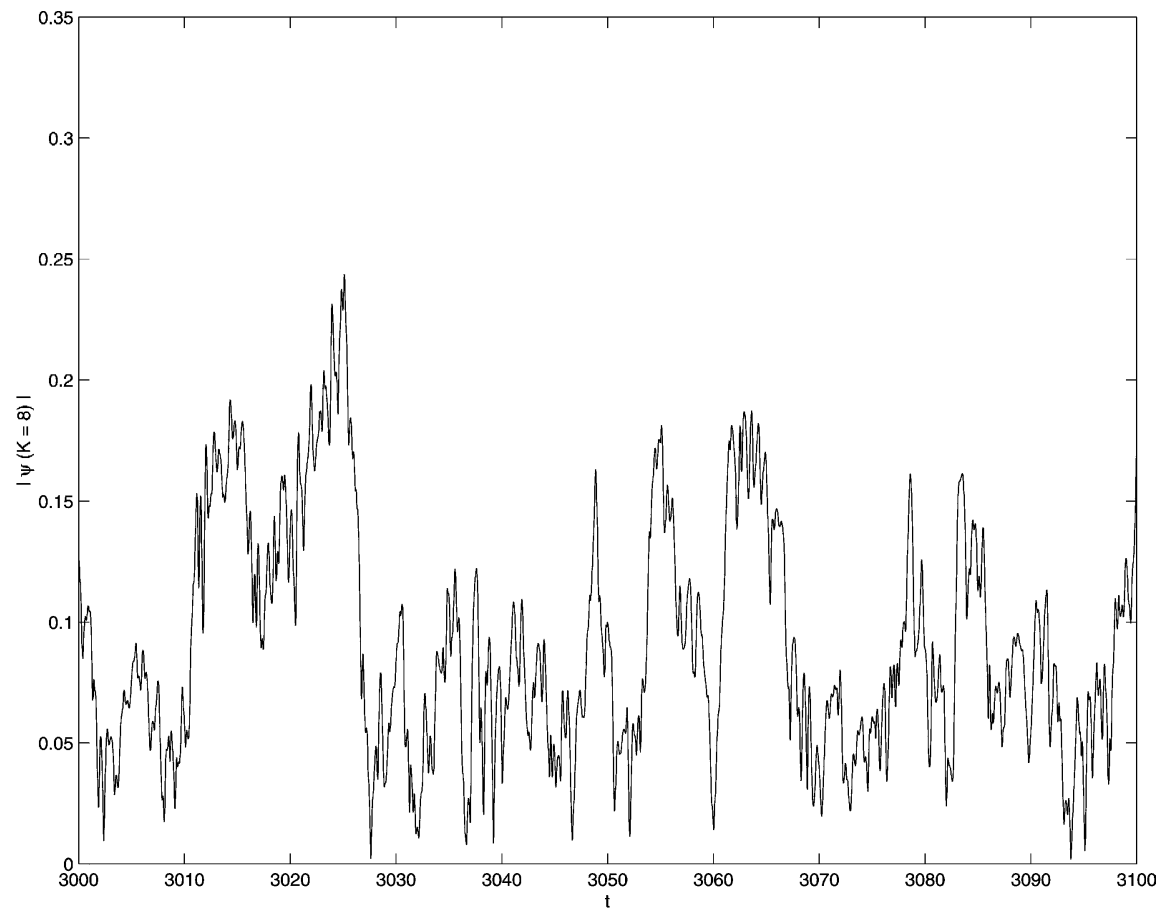

Fig. 2. $\beta=0, \lambda=-1$. Amplitude of the mode $k=8$ vs. time. 


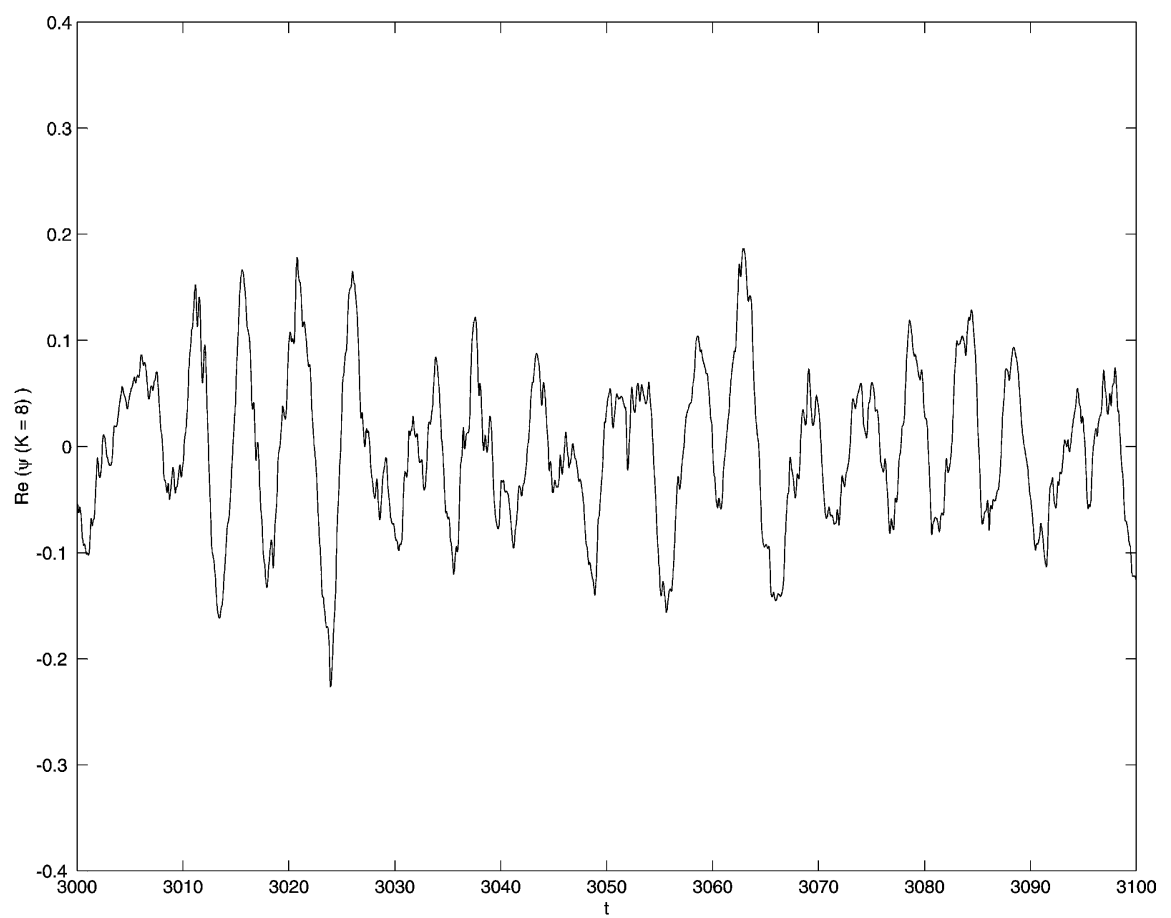

Fig. 3. $\beta=0, \lambda=-1$. Time evolution of the real part of the amplitude for the mode $k=8$.

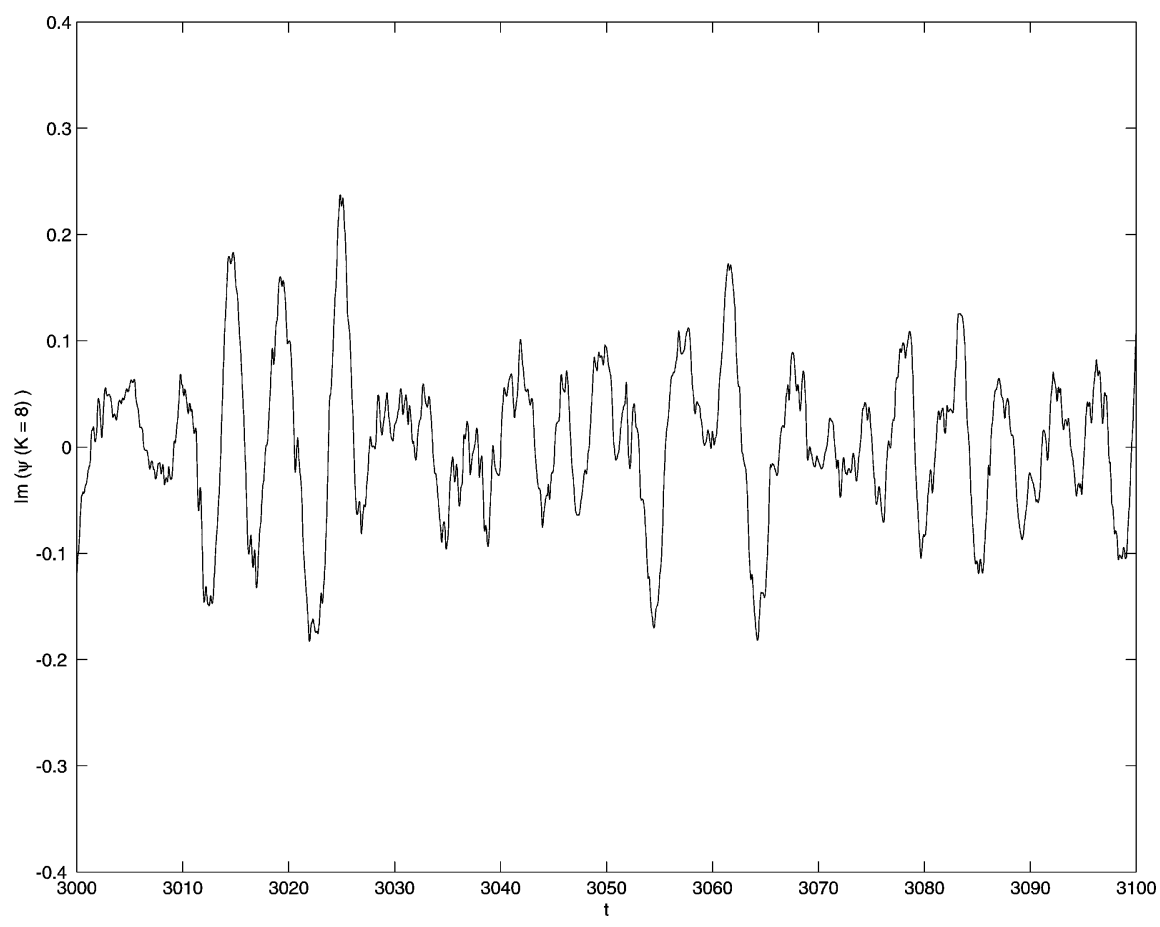

Fig. 4. $\beta=0, \lambda=-1$. Time evolution of the imaginary part of the amplitude for the mode $k=8$. 


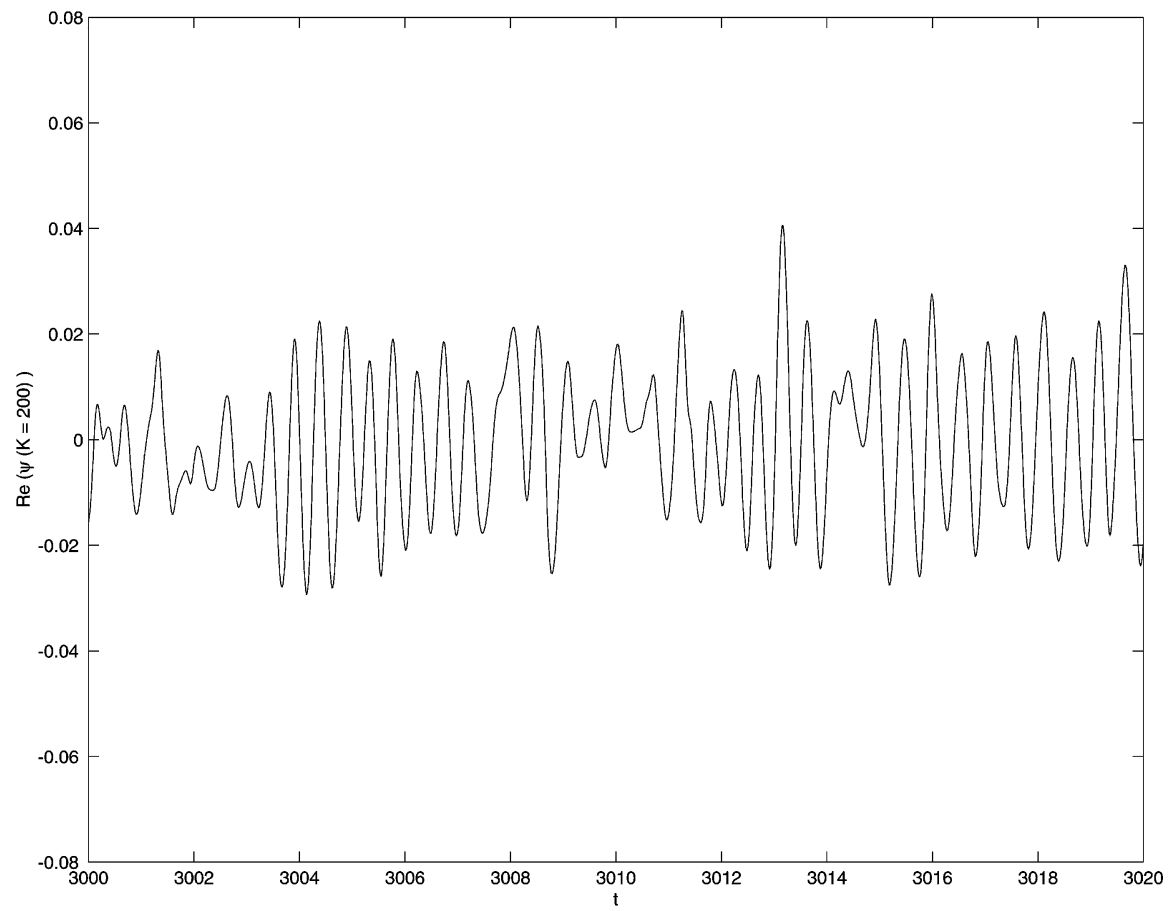

Fig. 5. $\beta=0, \lambda=-1$. Time evolution of the real part of the amplitude for the mode $k=200$ (time resolution $\tau=0.015$ ).

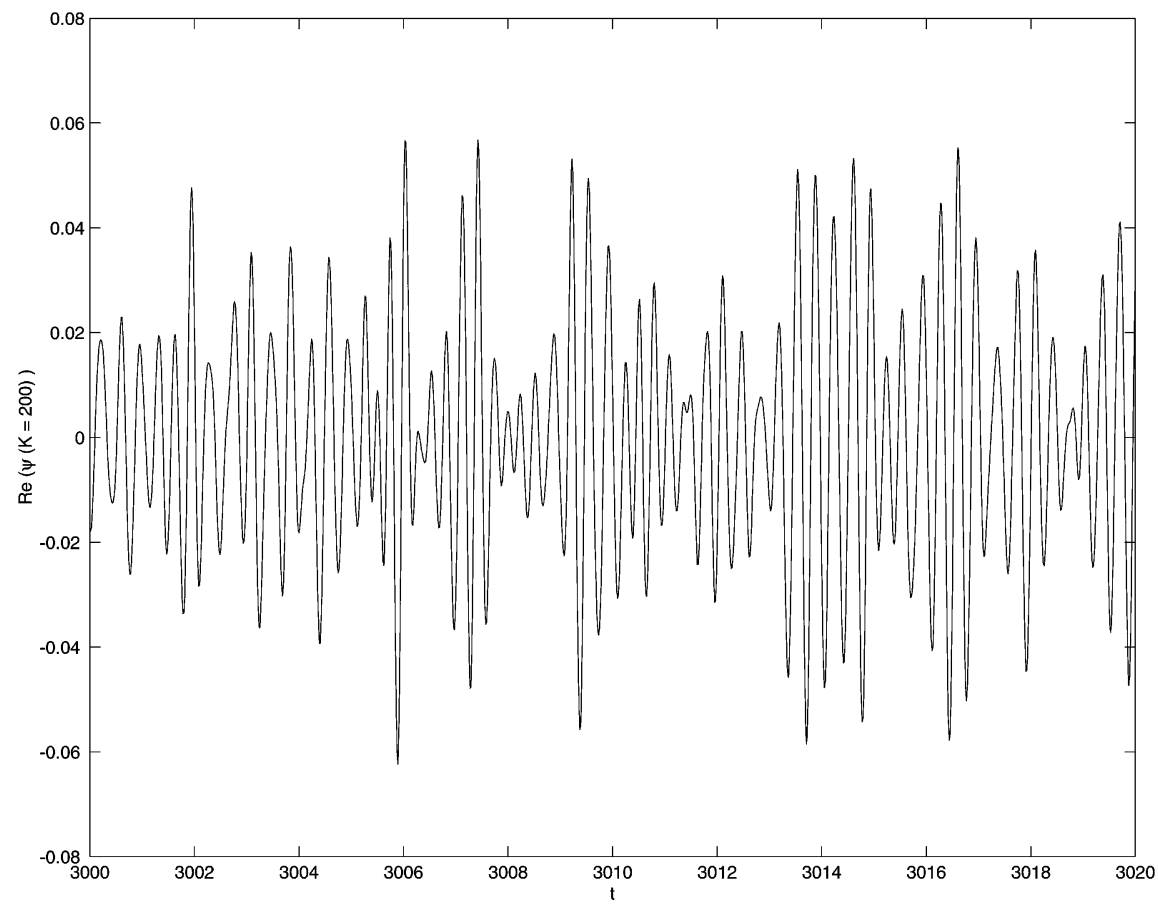

Fig. 6. $\beta=0, \lambda=+1$. Time evolution of the real part of the amplitude for the mode $k=200$ (time resolution $\tau=0.015$ ). 


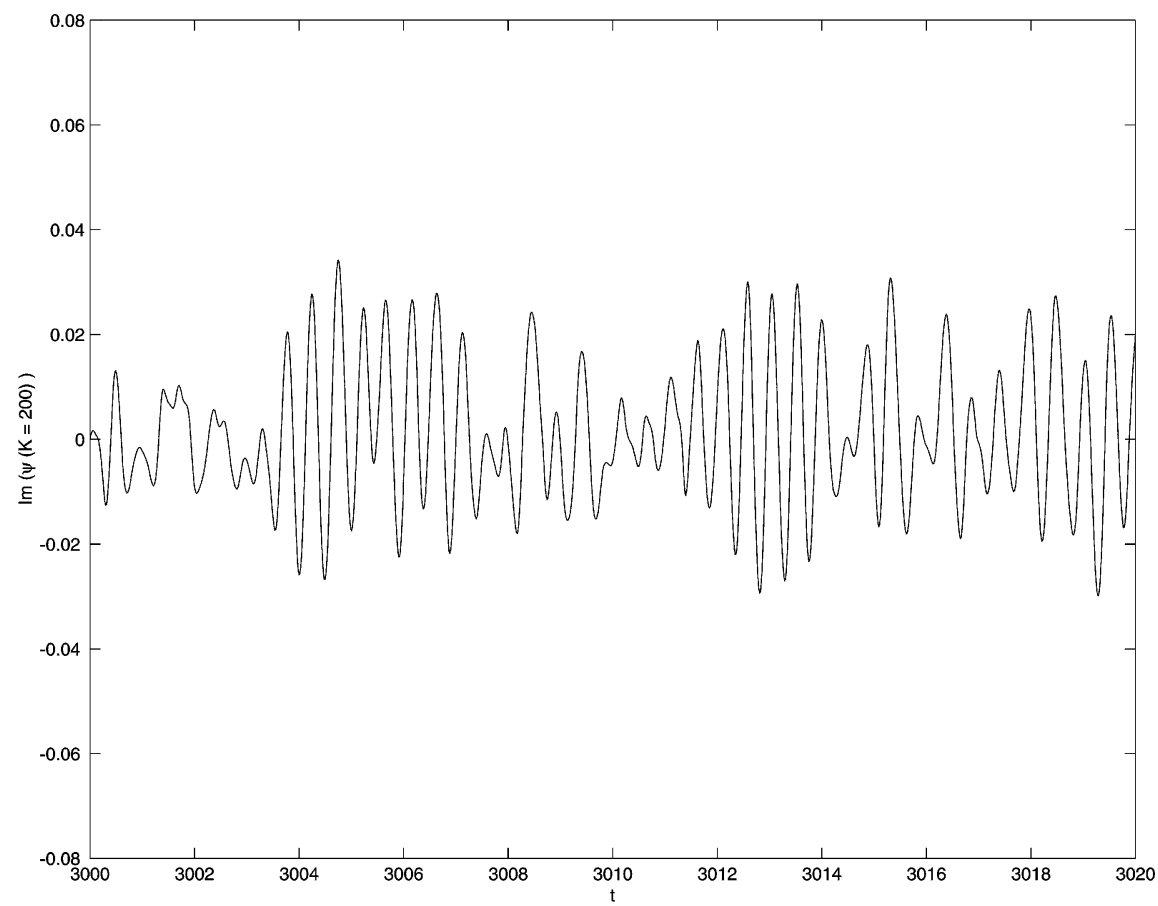

Fig. 7. $\beta=0, \lambda=-1$. Time evolution of the imaginary part of the amplitude for the mode $k=200$ (time resolution $\tau=0.015$ ).

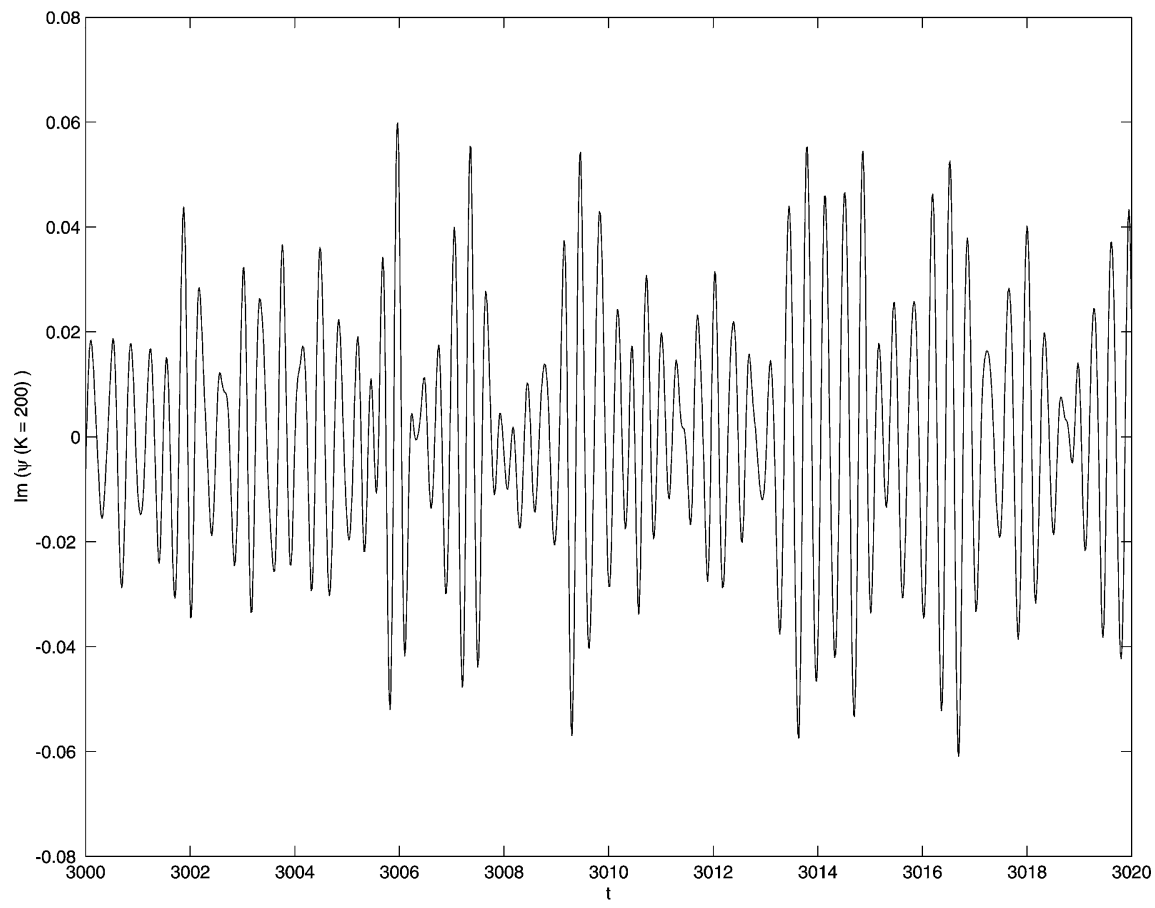

Fig. 8. $\beta=0, \lambda=+1$. Time evolution of the imaginary part of the amplitude for the mode $k=200$ (time resolution $\tau=0.015$ ). 
V.E. Zakharov et al. / Physica D 152-153 (2001) 573-619

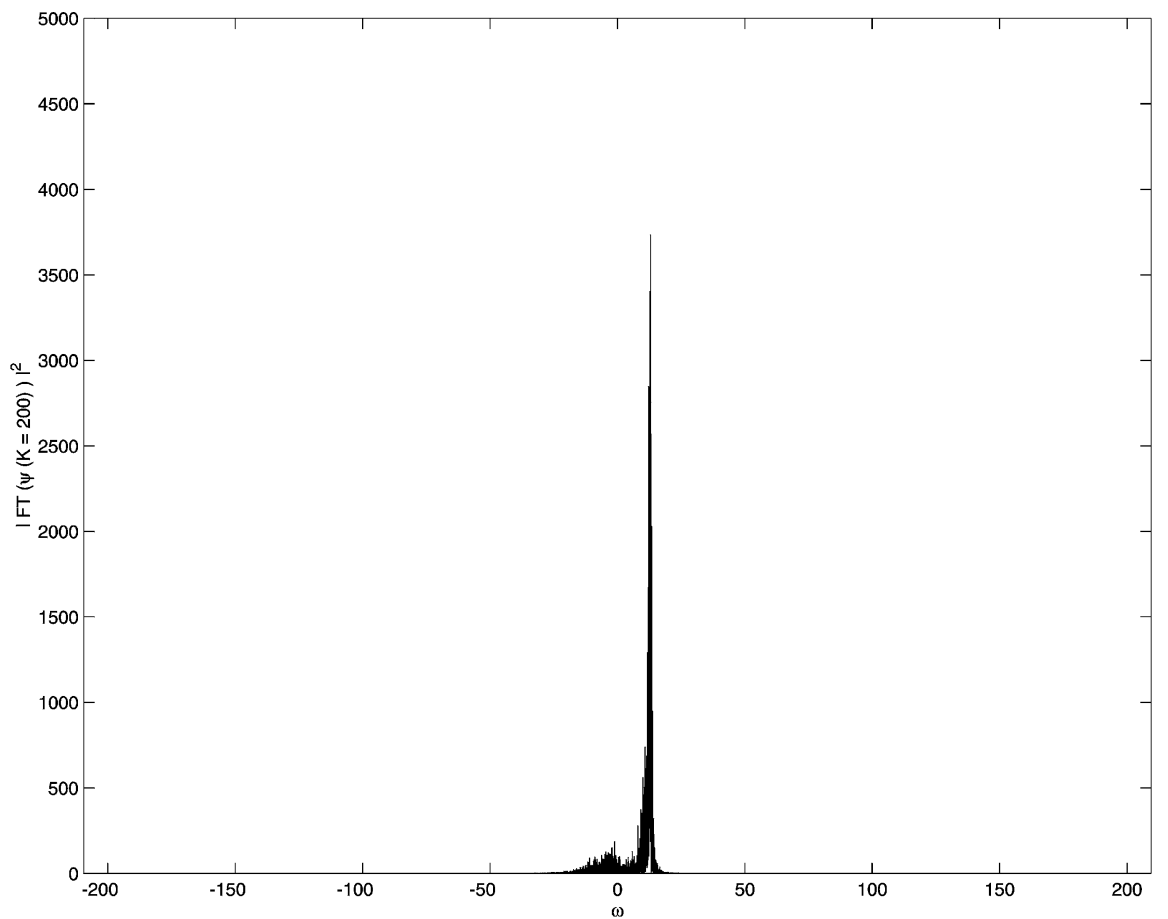

Fig. 9. $\beta=0, \lambda=-1$. Square amplitude of the FT for the mode $k=200$ vs. frequency (time resolution $\tau=0.015$ ).

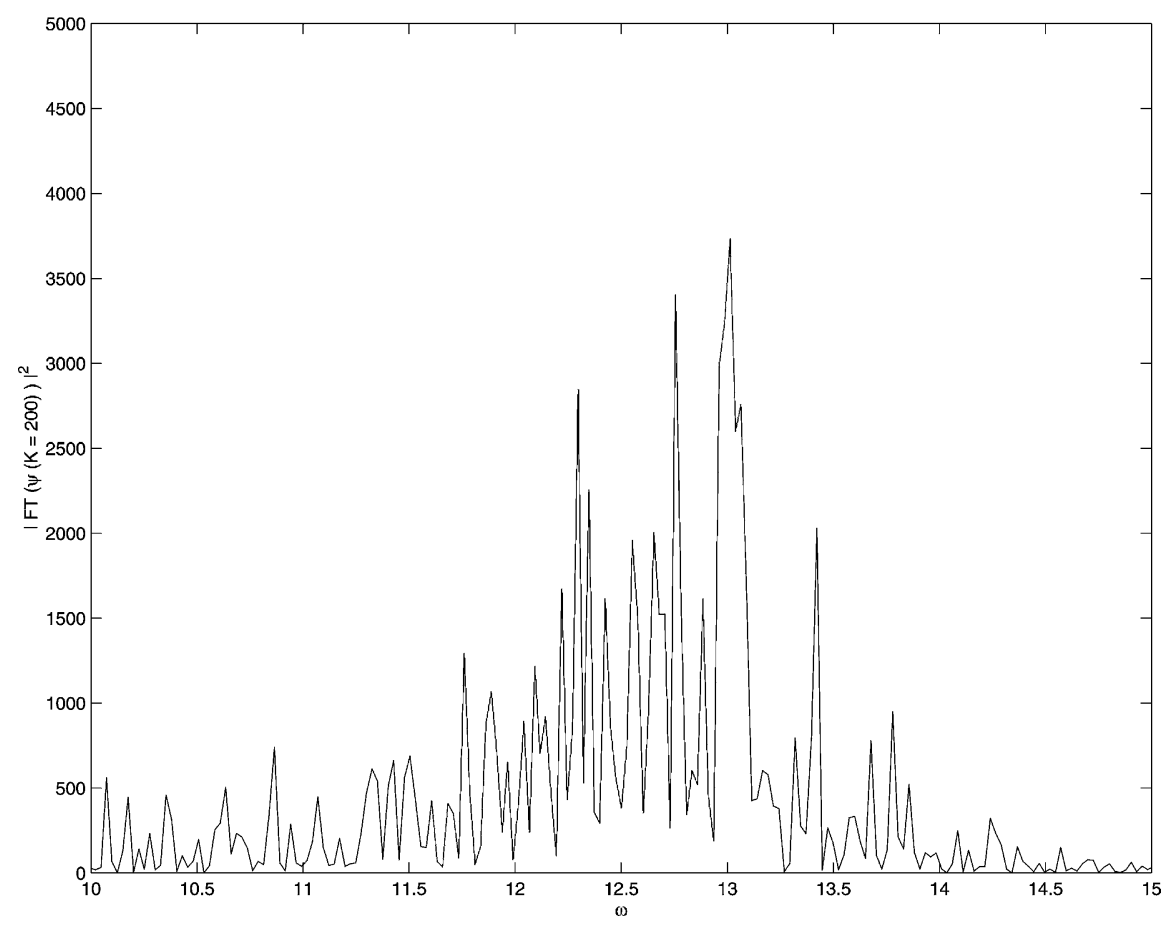

Fig. 10. $\beta=0, \lambda=-1$. Same as before but with a zoom on a smaller frequency window. 


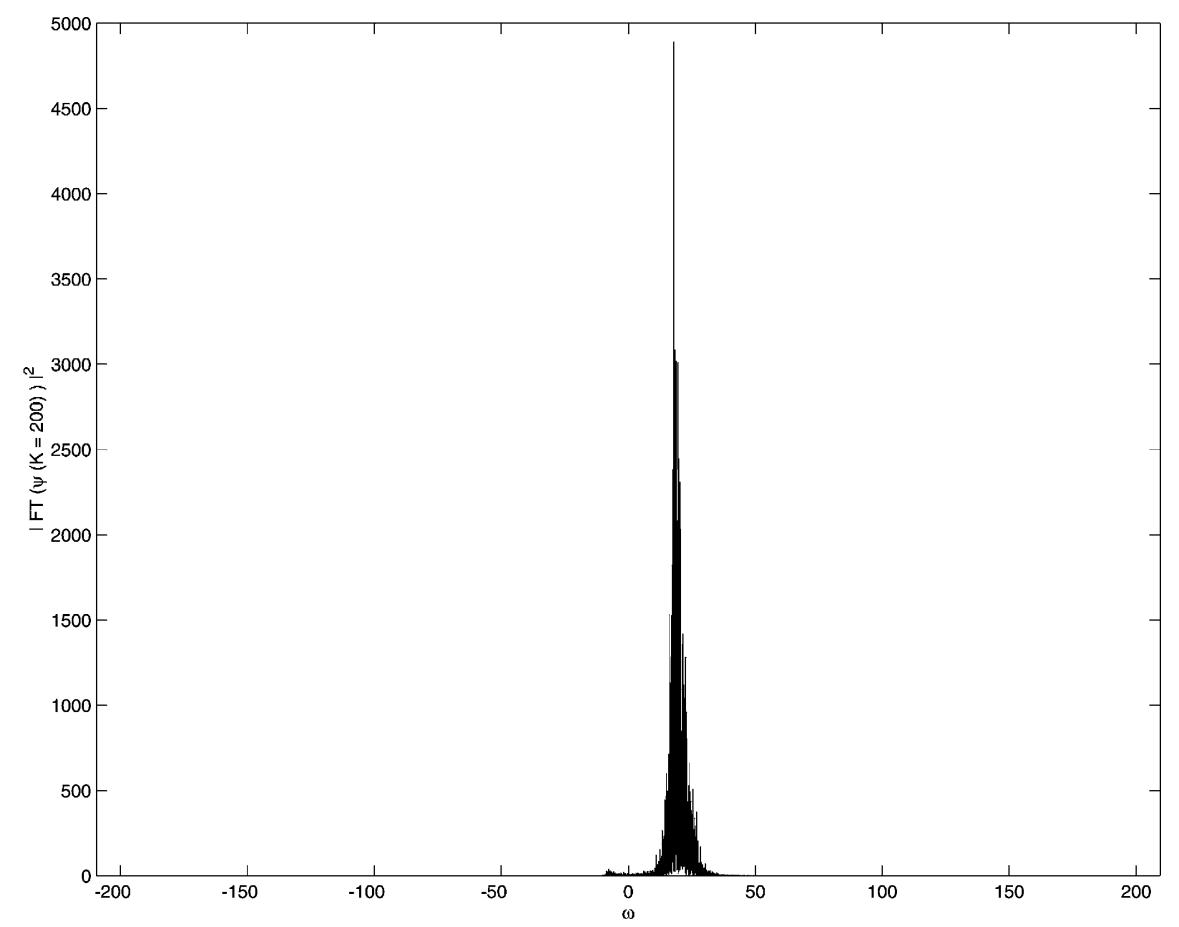

Fig. 11. $\beta=0, \lambda=+1$. Square amplitude of the FT for the mode $k=200 \mathrm{vs}$. frequency (time resolution $\tau=0.015$ ).

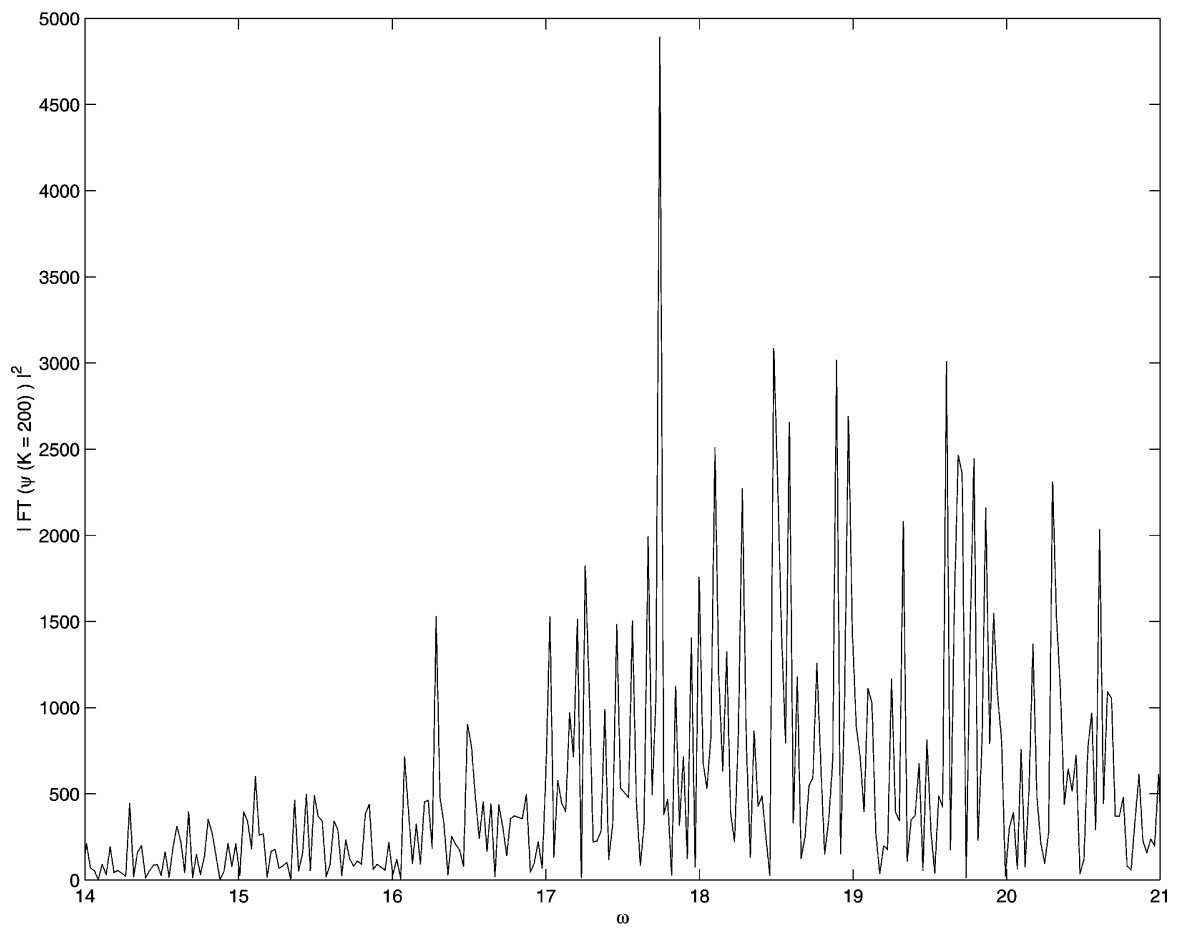

Fig. 12. $\beta=0, \lambda=+1$. Same as before but with a zoom on a smaller frequency window. 


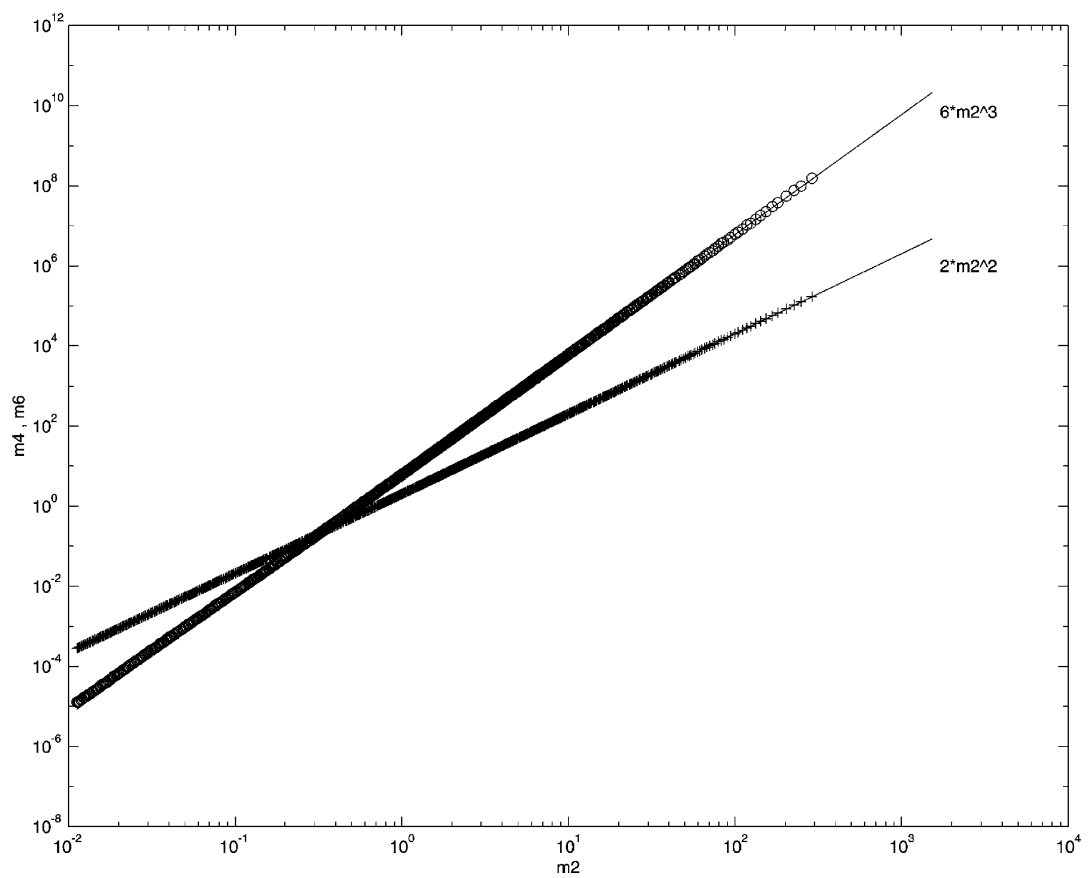

Fig. 13. $\beta=0, \lambda=+1$. Fourth-order (crosses) and sixth-order (circles) moments as functions of the second-order moments. The straight lines are the fitted Gaussian laws.

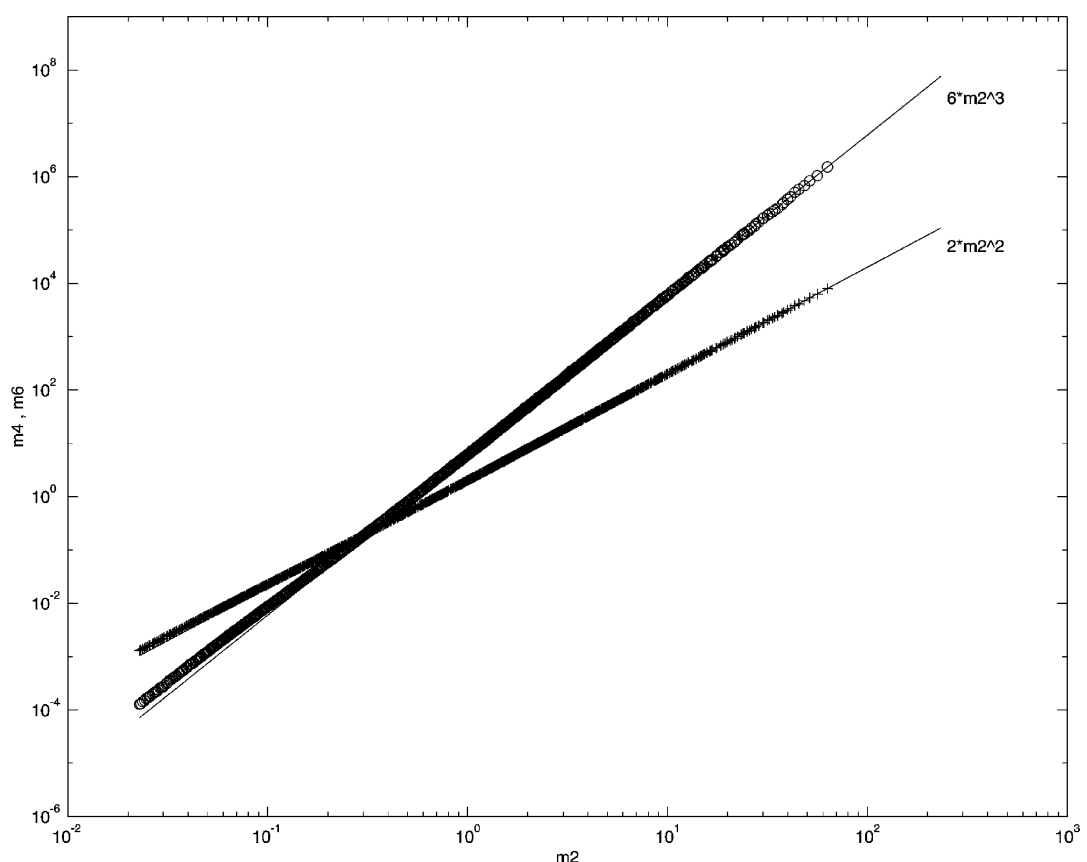

Fig. 14. $\beta=0, \lambda=-1$. Fourth-order (crosses) and sixth-order (circles) moments as functions of the second-order moments. The straight lines are the fitted Gaussian laws. 


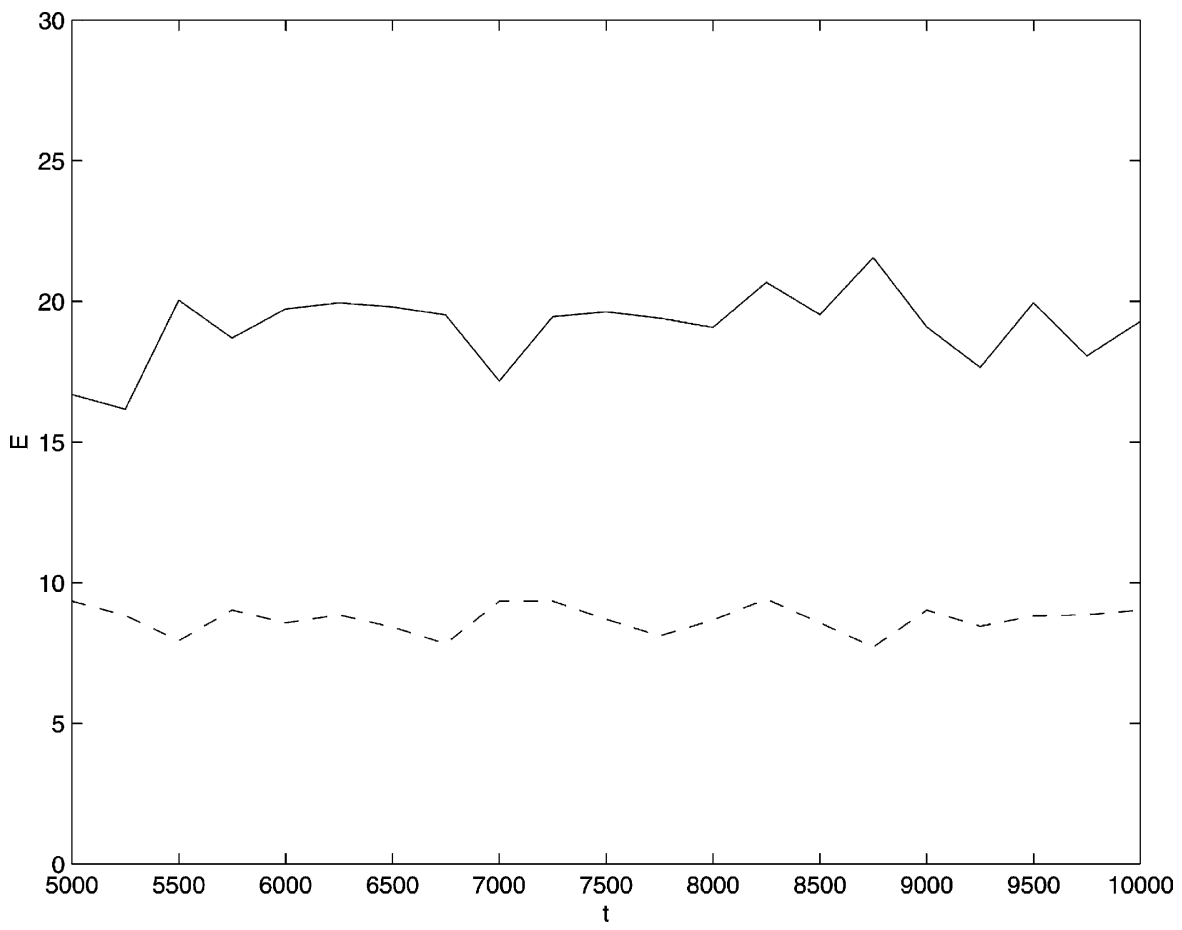

Fig. 15. $\beta=0$. Quadratic energy vs. time. $\lambda=+1$ (solid line), $\lambda=-1$ (dashed line).

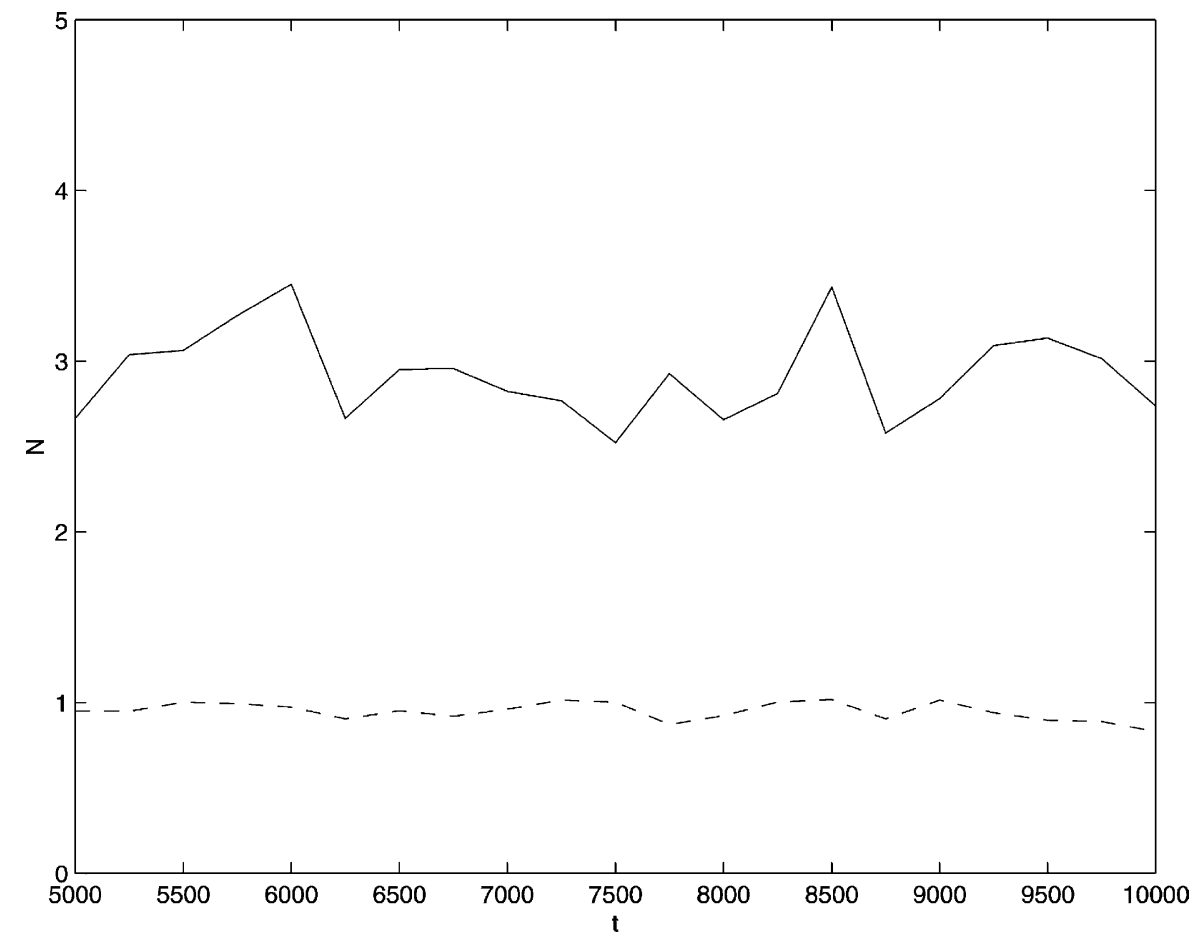

Fig. 16. $\beta=0$. Number of particles vs. time. $\lambda=+1$ (solid line), $\lambda=-1$ (dashed line). 


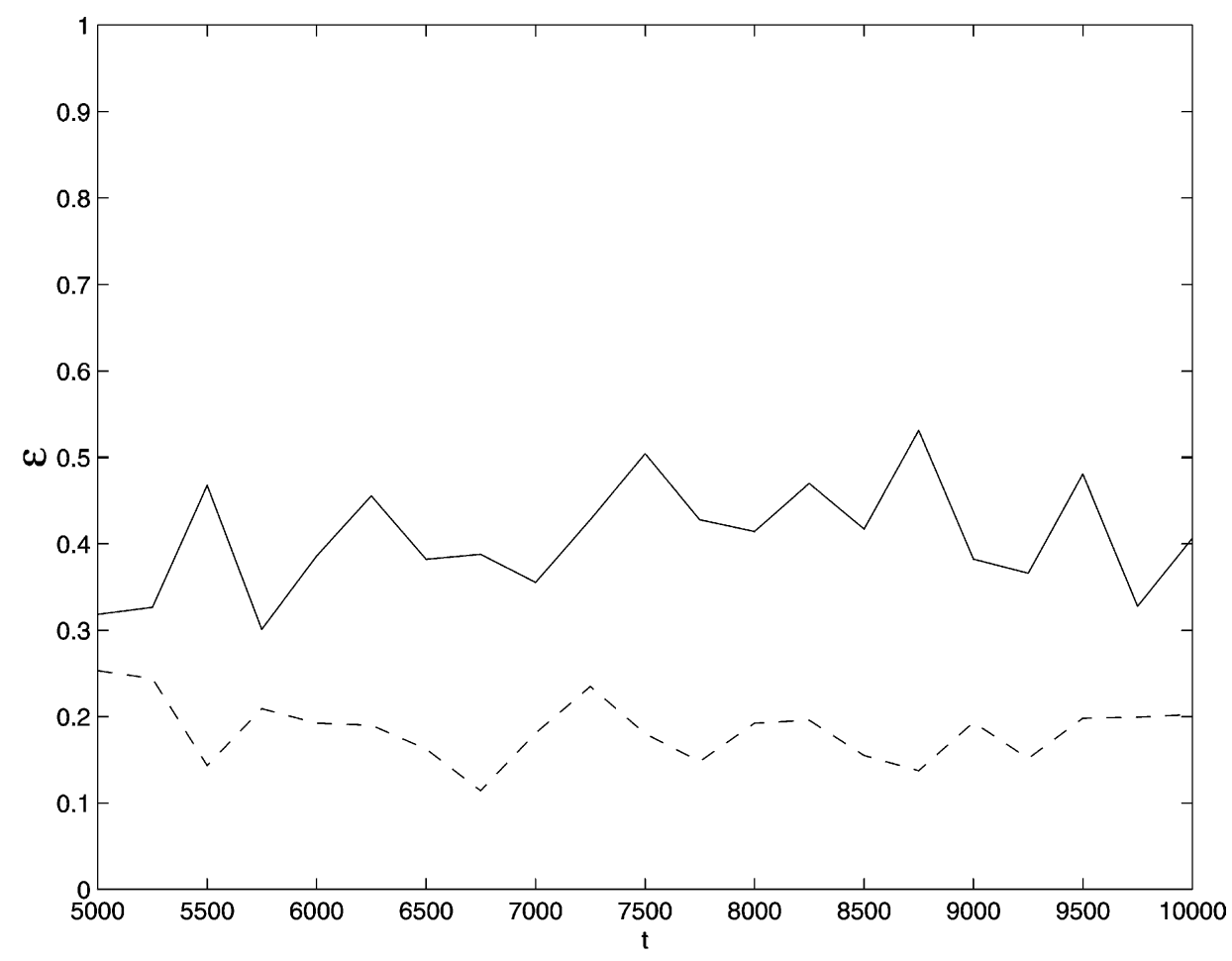

Fig. 17. $\beta=0$. Average nonlinearity $\epsilon=\left|H_{\mathrm{NL}} / H_{\mathrm{L}}\right|$ vs. time. $\lambda=+1$ (solid line), $\lambda=-1$ (dashed line).

obviously sees that the systems have already reached the steady state. Their energies moderately fluctuate about mean values which are $E \simeq 19(\lambda=+1)$ and $E \simeq 9(\lambda=-1)$. This significant difference with respect to the sign of $\lambda$ is quite unexpected from the viewpoint of the WT theory since the same rate of forcing is imposed in both systems. We can make the same remarks about the evolution of the number of particles $N$. In Fig. 16, the mean values stay near $N \simeq 3(\lambda=+1)$ and $N \simeq 1(\lambda=-1)$ so that their relative difference is even bigger than for $E$. Fluctuations also spread much more in the case $\lambda=+1$.

In Fig. 17, the stationarity as well as the gap between both signs of $\lambda$ are verified again in the time evolution of the average nonlinearity $\epsilon$. We define the average nonlinearity in the system as the ratio of the nonlinear part to the linear part of the Hamiltonian $\epsilon=\left|H_{\mathrm{NL}} / H_{\mathrm{L}}\right|$, each part being calculated over the whole field. Of course, this definition does not really make sense when external forces are applied but it provides a relatively good estimation of the level of nonlinearity once the systems reach the steady state. Note here that the mean values $\epsilon \simeq 0.4(\lambda=+1)$ and $\epsilon \simeq 0.2(\lambda=-1)$ are relatively small. Thus, the condition of small nonlinearity required by the theory holds for both systems. This conclusion is also supported by comparing Figs. 10 and 12. It is seen that the difference of frequencies caused by nonlinearity is relatively small. We point out that in our numerical

Table 2

Time-averaged values of the wave action, quadratic energy and corresponding fluxes in the stationary state, $\alpha=\frac{1}{2}, \beta=0$

\begin{tabular}{lllllll}
\hline$\lambda$ & $N$ & $E$ & $Q^{-}$ & $Q^{+}$ & $P^{-}$ & $P^{+}$ \\
\hline+1 & 3 & 19 & 0.1957 & 0.0090 & 0.276 & 0.258 \\
-1 & 1 & 9 & 0.0098 & 0.0478 & 0.014 & 1.430 \\
\hline
\end{tabular}



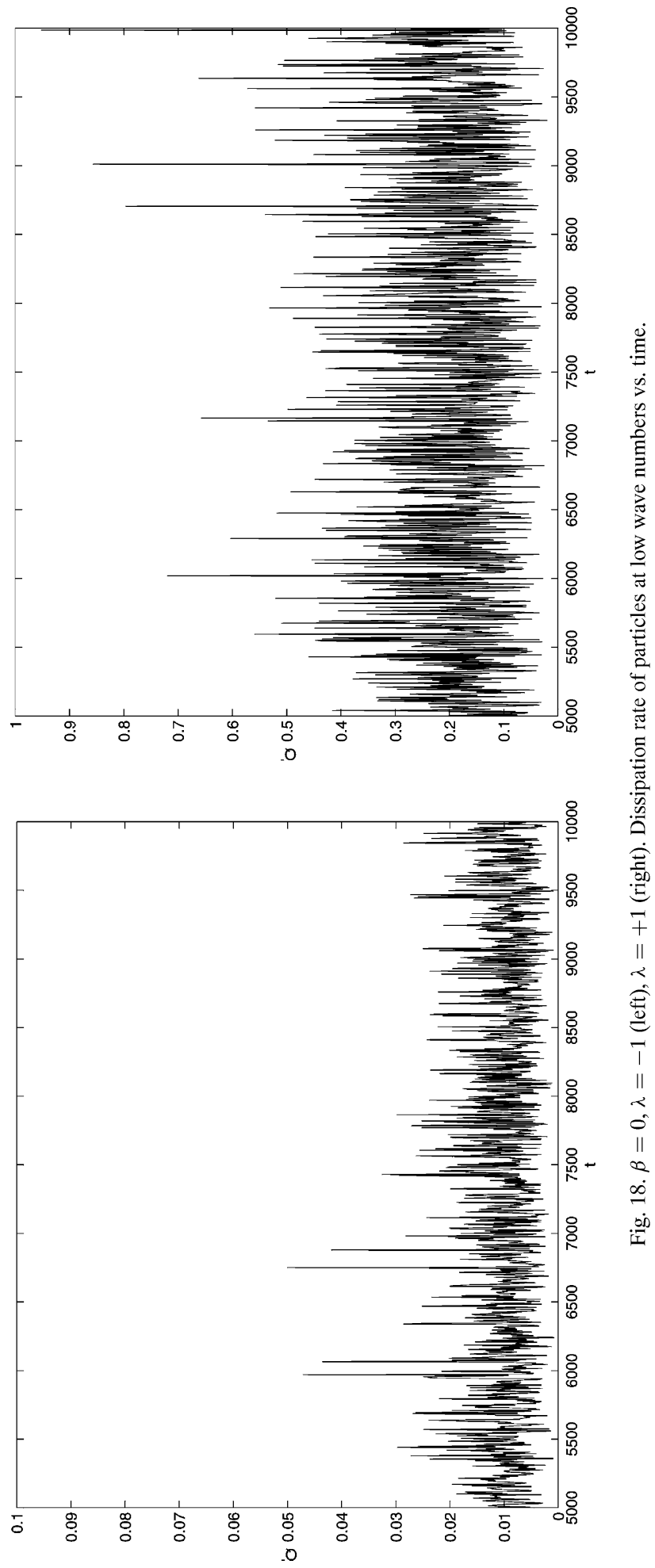


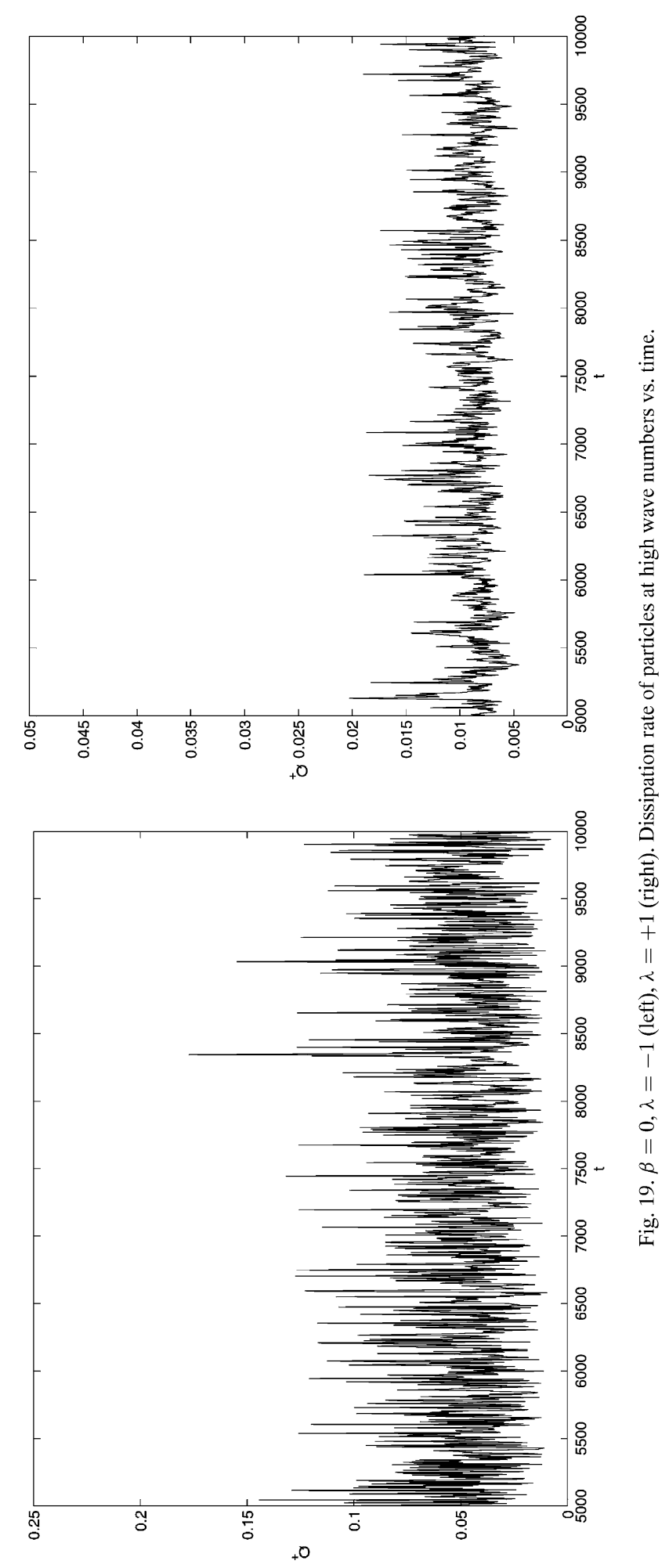



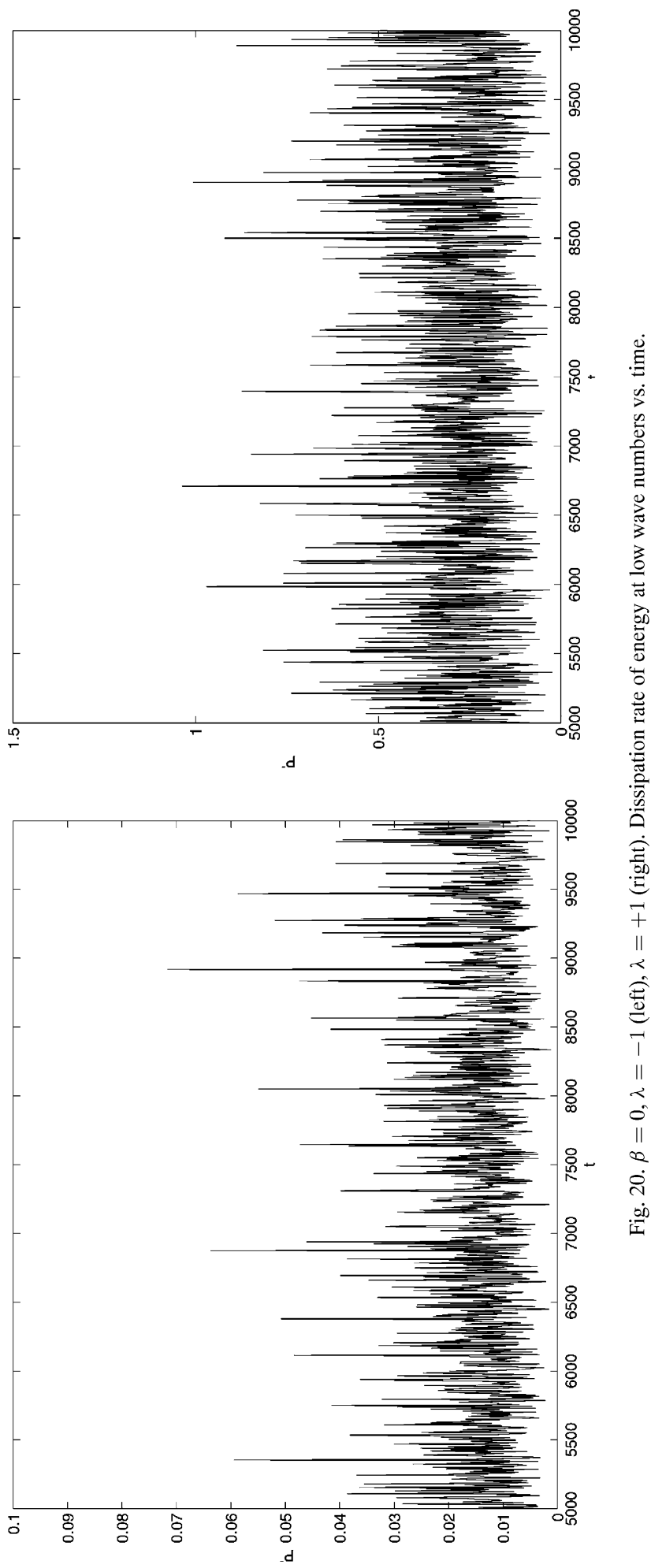


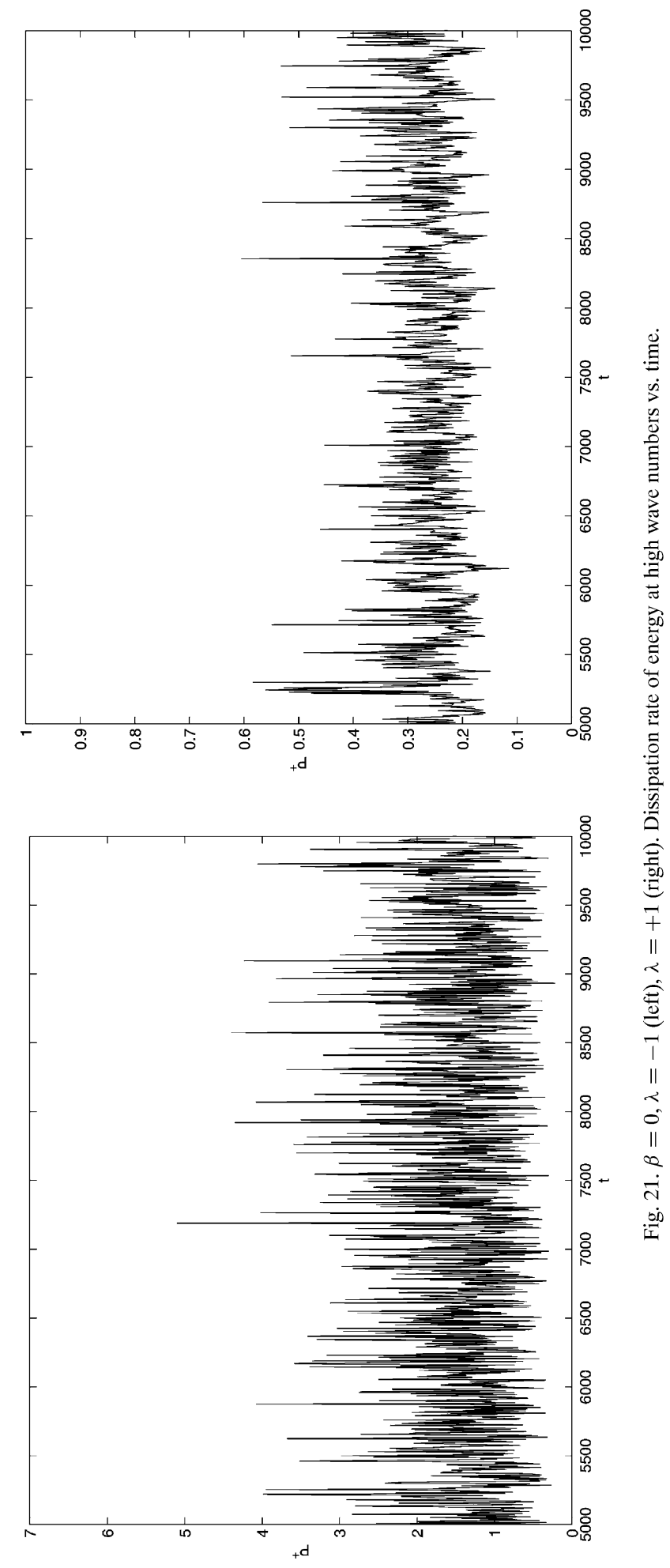



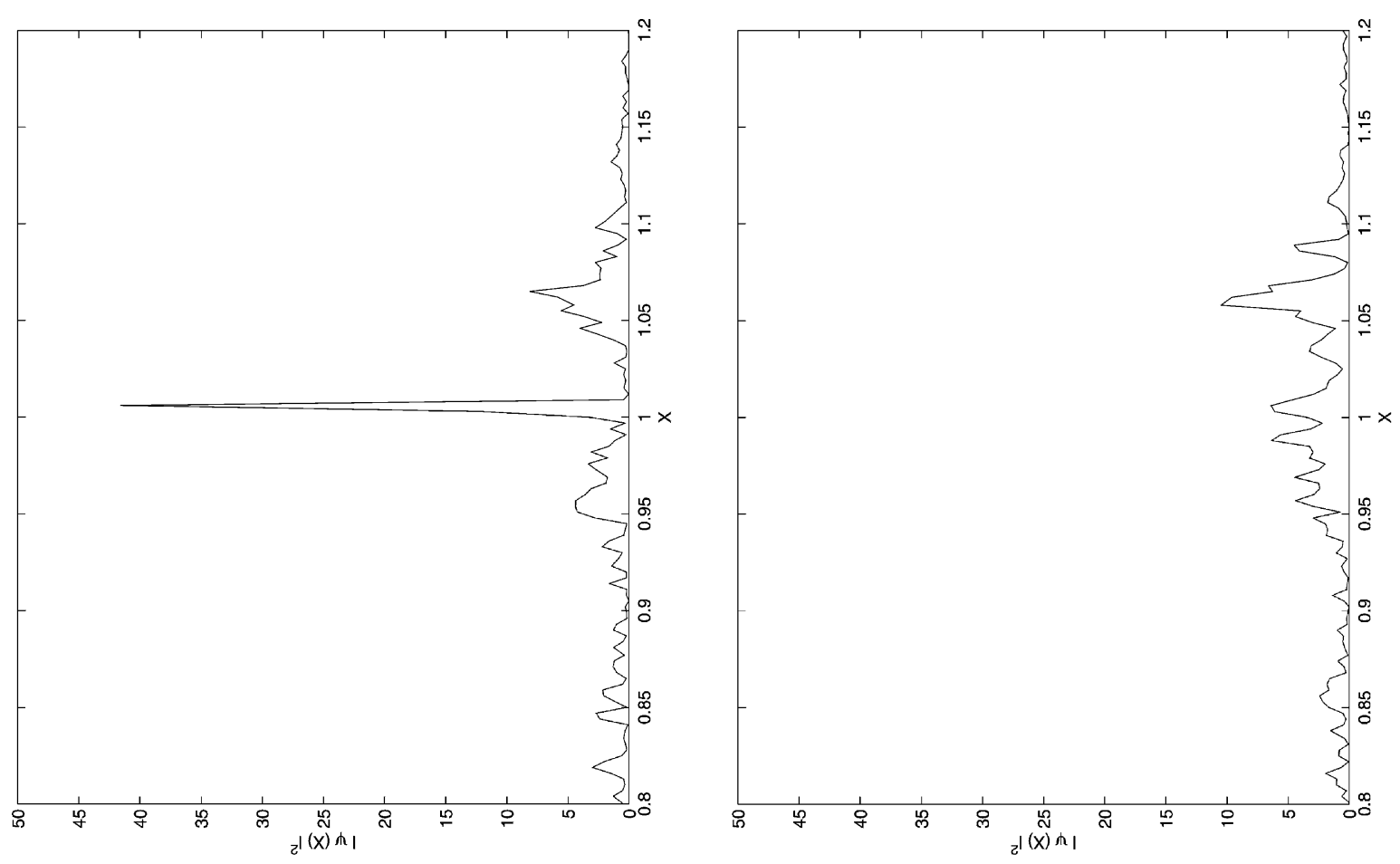

घี
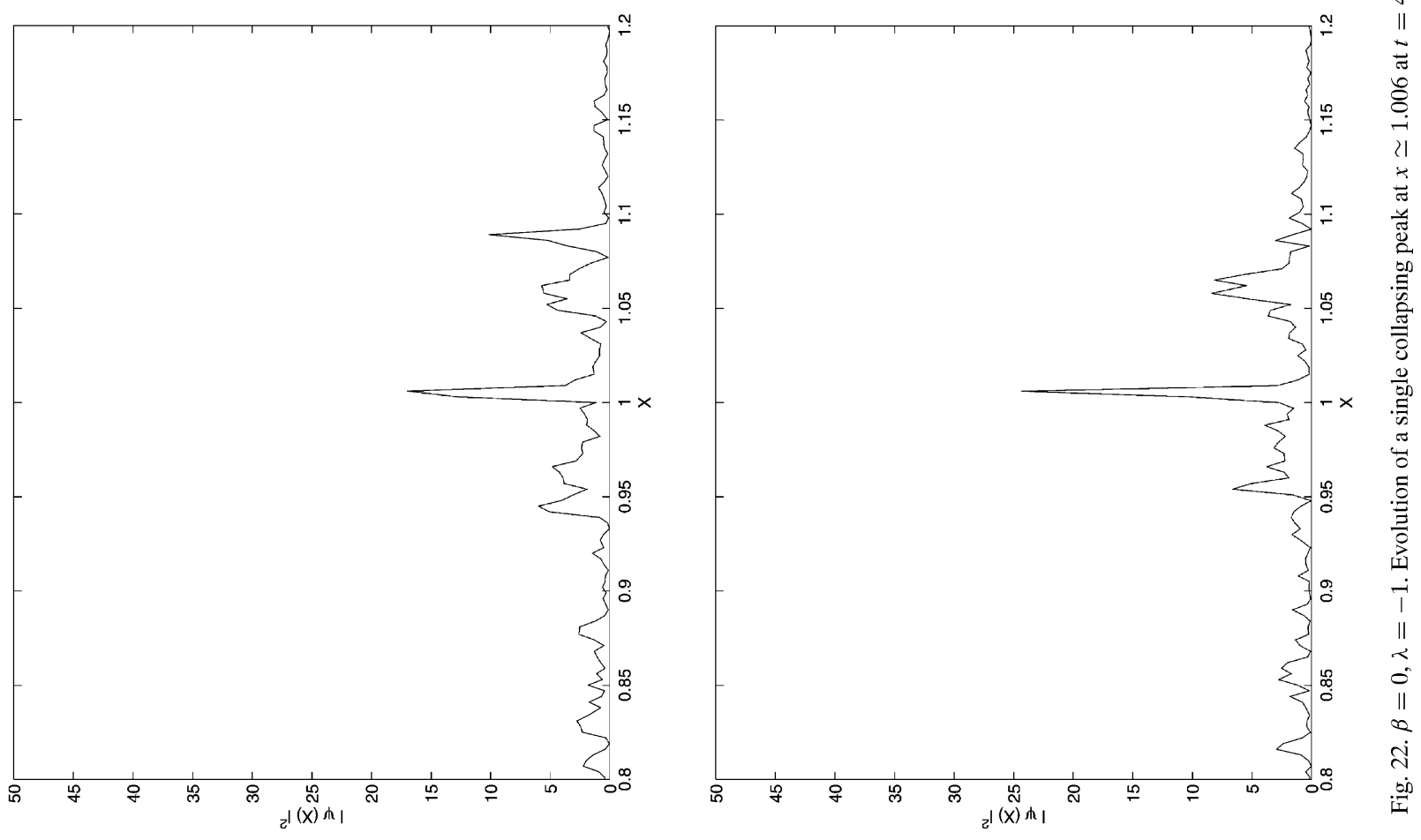


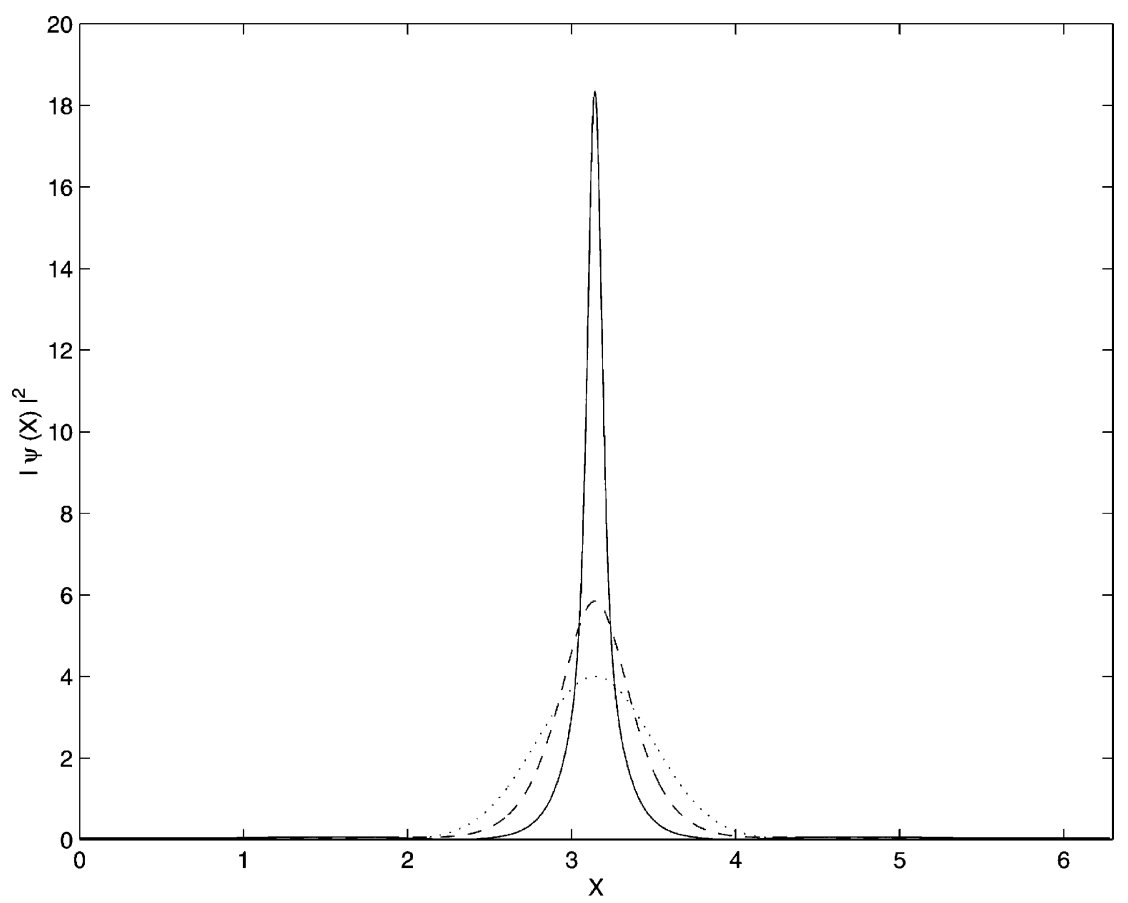

Fig. 23. $\beta=0, \lambda=-1$. Evolution towards a collapsing peak of the isolated solution for the initial amplitude $\psi_{0}=2$. Dotted line $t=0$, dashed line $t=0.55$, solid line $t=1.1$.

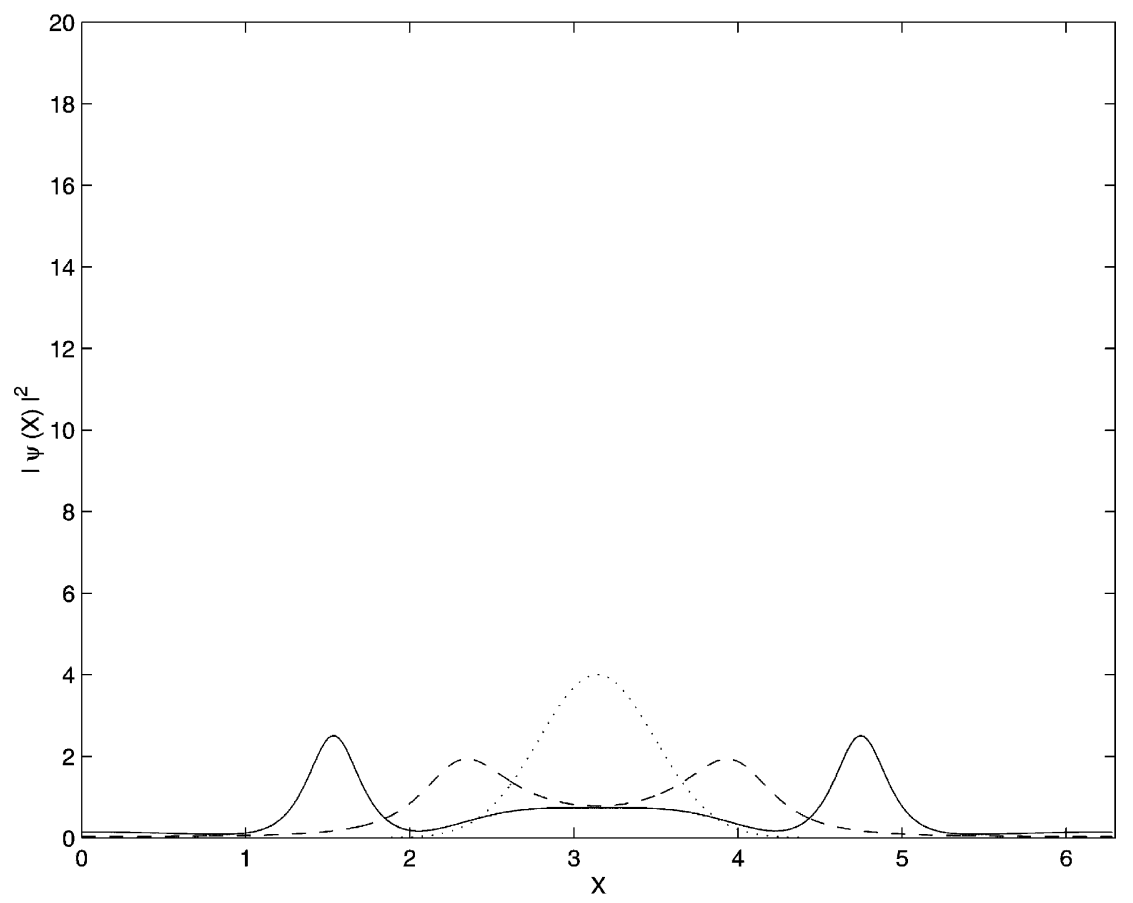

Fig. 24. $\beta=0, \lambda=+1$. Evolution towards decay of the isolated solution for the initial amplitude $\psi_{0}=2$. Dotted line $t=0$, dashed line $t=1.65$, solid line $t=3.85$. 


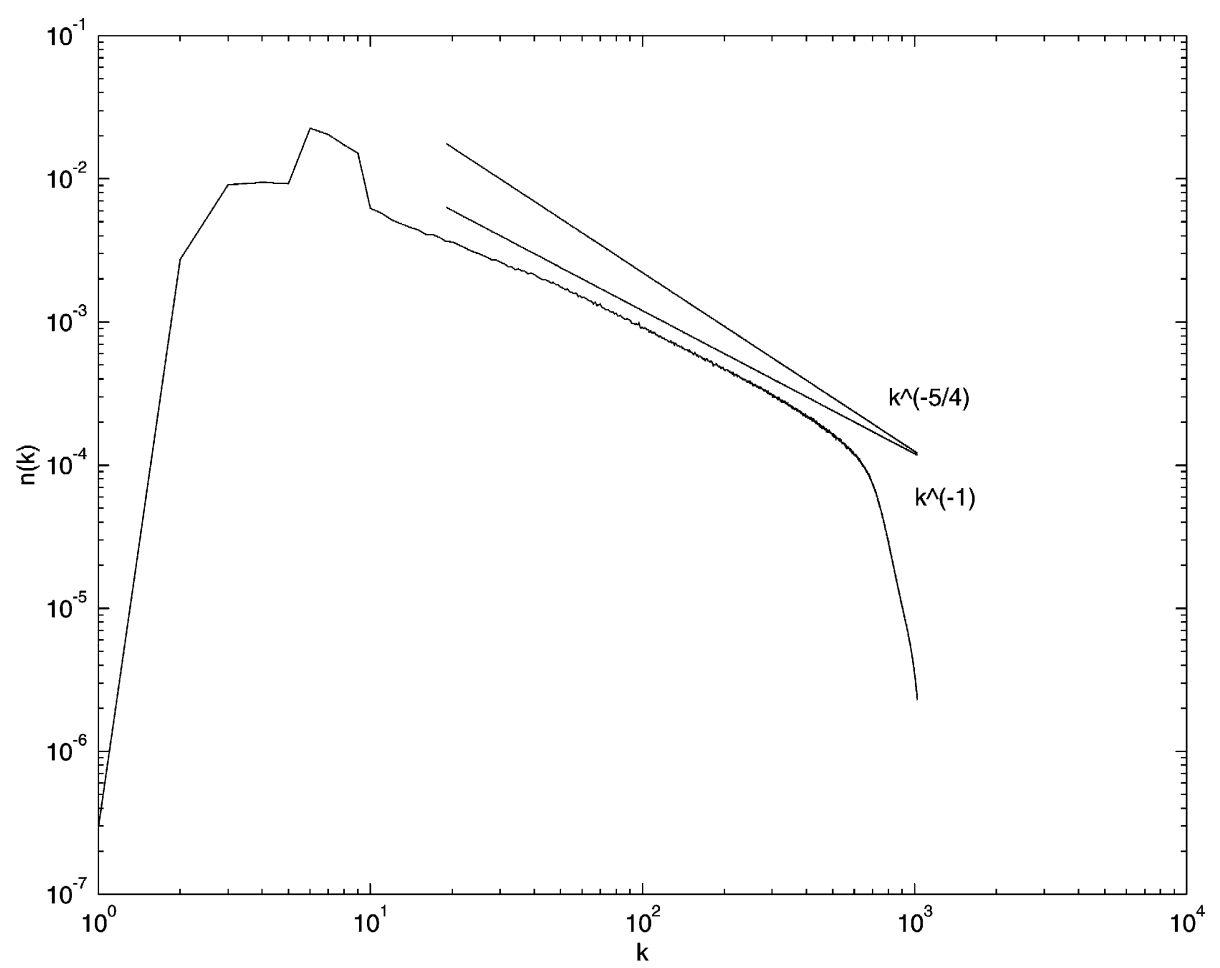

Fig. 25. $\beta=0, \lambda=-1$. Computed spectrum vs. wave number. The theoretical slopes are shown as well $\left(k^{-1}\right.$ for WT and $k^{-5 / 4}$ for MMT).

experiments $\epsilon$ could not be taken too small (i.e. $\epsilon \leq 10^{-3}$ ) for two reasons. First, the nonlinear turnover time grows longer and the energy flux is too weak to act effectively. Second, one may catch the undesirable frozen turbulence [13] due to the disappearance of quasi-resonances. One should note that, in general, frozen turbulence arises more easily in one-dimensional problems due to fewer degrees of freedom than in higher-dimensional problems.

The difference between the cases $\lambda= \pm 1$ is especially conspicuous if one considers the dissipation rates of particles and quadratic energy for small wave numbers

$$
Q^{-}=2 \int_{k<k_{\mathrm{f}}} v^{-}|k|^{-d^{-}}\left|\hat{\psi}_{k}\right|^{2} \mathrm{~d} k, \quad P^{-}=2 \int_{k<k_{\mathrm{f}}} v^{-}|k|^{-d^{-}} \omega(k)\left|\hat{\psi}_{k}\right|^{2} \mathrm{~d} k,
$$

and for large wave numbers

$$
Q^{+}=2 \int_{k>k_{\mathrm{f}}} v^{+}|k|^{d^{+}}\left|\hat{\psi}_{k}\right|^{2} \mathrm{~d} k, \quad P^{+}=2 \int_{k>k_{\mathrm{f}}} v^{+}|k|^{d^{+}} \omega(k)\left|\hat{\psi}_{k}\right|^{2} \mathrm{~d} k,
$$

where $k_{\mathrm{f}}$ is the characteristic wave number of forcing. Figs. 18-21 represent the time evolution of these quantities and their time-averaged values are collected in Table 2.

One can see that the case $\lambda=+1$ quantitatively fits WT theory. Indeed, in this case $Q^{+} / Q^{-} \simeq 0.046 \ll 1$ and $P^{+} / P^{-} \simeq 0.94$. But in the case of negative nonlinearity $\lambda=-1$ the situation is opposite. In this case $Q^{+} / Q^{-} \simeq 4.9$ and $P^{+} / P^{-} \simeq 102$ which means that most of both quadratic energy and particles are transported to high frequencies. 


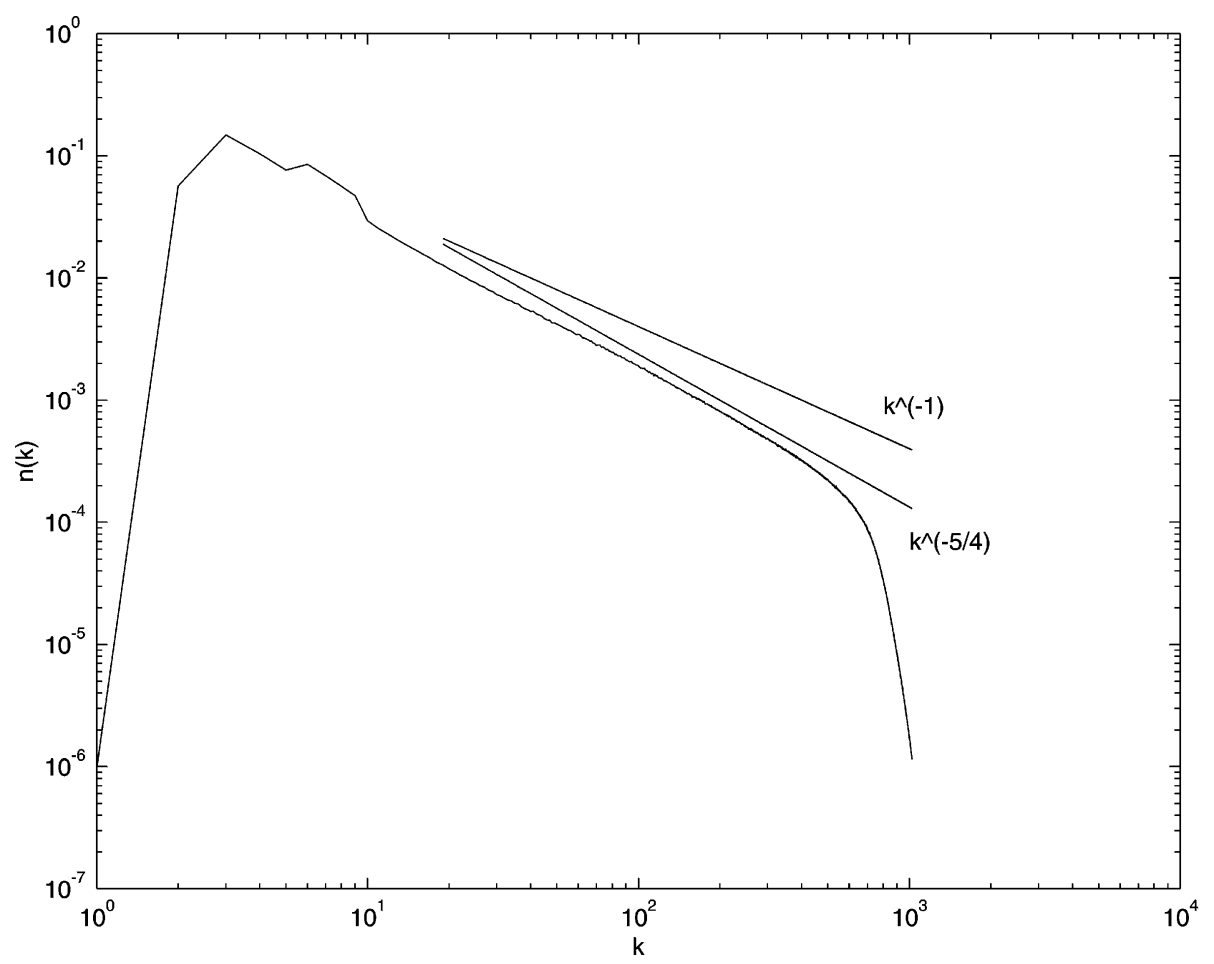

Fig. 26. $\beta=0, \lambda=+1$. Computed spectrum vs. wave number. The theoretical slopes are shown as well $\left(k^{-1}\right.$ for WT and $k^{-5 / 4}$ for MMT).

Comparison of the turbulence levels and fluxes of particles $Q^{+}$for both signs of nonlinearity leads to a paradoxal result. At $\lambda=-1$ the total number of particles is three times less than at $\lambda=+1$, while the dissipation rate of particles is higher by one order of magnitude. It can be explained only by the presence in this case of a much more powerful mechanism of nonlinear interactions, which provides very fast wave particles transport to high frequencies. In our opinion, this mechanism is wave collapse, studied theoretically in Section 7. Sporadic collapsing events developing on top of the WT background could send most of particles to high wave numbers without violation of energy conservation, because in each self-similar collapse structure the amount of total energy is zero.

We observed such collapsing events in our numerical experiments. Fig. 22 displays the collapse event taking place at the point $x=1.006$ at time $t=5000.19$. One can conjecture that the collapses are described by self-similar solutions. For such solutions $H \equiv 0$. It means that the collapse can carry particles to high frequencies, without carrying any energy at that time! As far as the Hamiltonian is the difference of quadratic and quartic terms and both of them go to infinity, it becomes possible to explain the apparent contradictions of the dissipation rates.

The hypothesis related to the prevailing role of collapses at $\lambda=-1$ is corroborated by the following facts:

1. Intermittency in dissipation rates of quadratic energy and particles for $\lambda=-1$ is much higher than for $\lambda=+1$ in the region of large wave numbers. This intermittency can be explained by outbursts of dissipation when wave collapses occur.

2. The analysis of time FTs of separate harmonics (we take $k=200$ ) shows the presence of two components, see Fig. 9. The peak at $\omega \simeq 13$ corresponds to a linear wave with a moderate nonlinear shift of frequency. This is the "WT" component of the wave field. Another component is roughly symmetrical with respect to the 


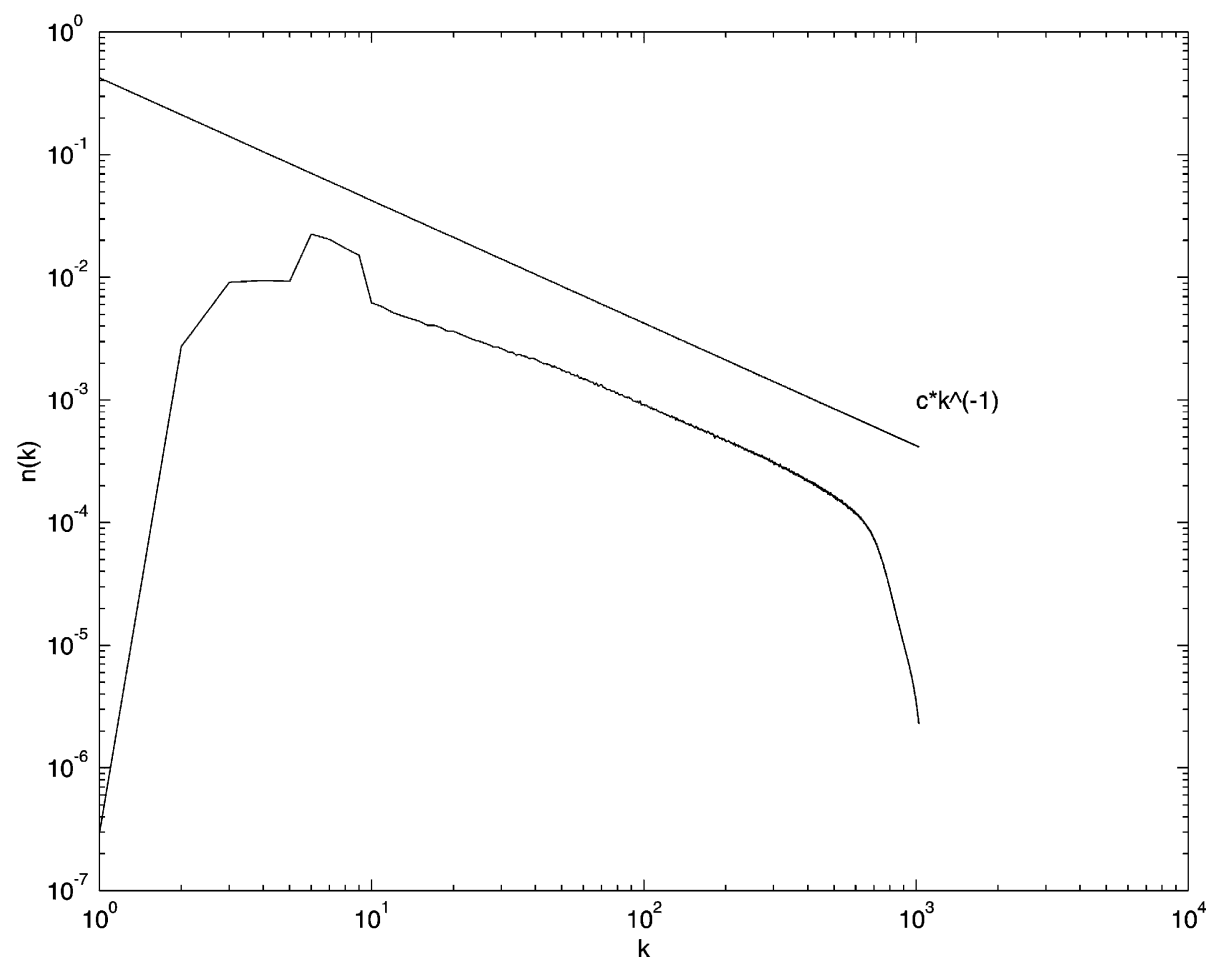

Fig. 27. $\beta=0, \lambda=-1$. Computed spectrum and WT spectrum vs. wave number. The WT spectrum (straight line) is given by $n(k)=c k^{-1}$ with $c=a P^{1 / 3} \simeq 0.42$.

reflection $\omega \rightarrow-\omega$ with the maximum at $\omega=0$. This is certainly a strongly nonlinear component which could be associated with wave collapses.

Another indication of the difference of the wave dynamics in the cases $\lambda=+1$ and $\lambda=-1$ follows from the following experiment. Figs. 23 and 24 show the early stages in the conservative evolution of the same isolated initial condition

$$
\psi(x)=\psi_{0} \mathrm{e}^{-(x-\pi)^{2} / 2 \sigma^{2}}, \quad \sigma=0.5
$$

In the case $\lambda=-1$, a sufficiently large initial condition collapses into a sharp spike, while in the case $\lambda=+1$ it decays. This experiment could serve as an evidence of the finite-time singularity formation for the case $\lambda=-1$.

Now, we discuss the stationary isotropic spectra of turbulence which are displayed in Figs. 25-28. We plotted on the same pictures the Kolmogorov spectra calculated by putting either $P=P^{+}=1.430(\lambda=-1)$ or $P=$ $P^{+}=0.258(\lambda=+1)$ and $a=0.376$ in Eq. (5.8). In Figs. 27 and 28, one can see that for both cases this spectrum provides a higher level of turbulence than the observed one. For $\lambda=-1$ this difference is almost of one order of magnitude. For $\lambda=+1$, the observed spectrum almost coincides with the WT one at low frequencies and then decays faster at higher wave numbers (approximately as MMT spectrum in Fig. 26).

It is interesting that for $\lambda=-1$ the high frequency asymptotics is fairly close to the one predicted by WT theory (Fig. 25). One can explain this fact as follows. In this case, the turbulence is the coexistence of collapsing events and WT. Collapses carry most of the fluxes of particles and quadratic energy to high frequencies. But their contribution 


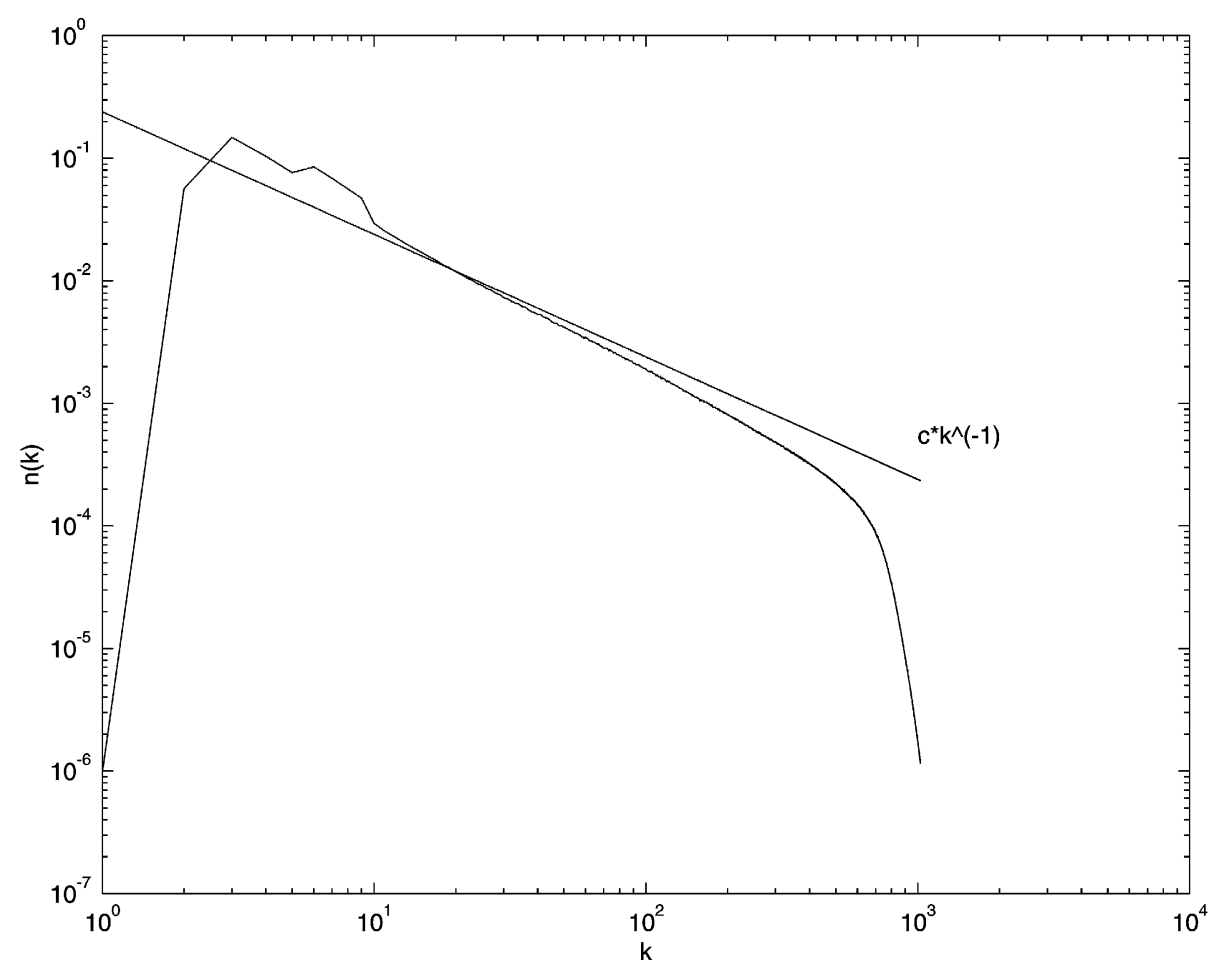

Fig. 28. $\beta=0, \lambda=+1$. Computed spectrum and WT spectrum vs. wave number. The WT spectrum (straight line) is given by $n(k)=c k^{-1}$ with $c=a P^{1 / 3} \simeq 0.24$.

to the high-frequency part of the spectrum is weak, because they produce Phillips-type spectra, decaying very fast as $k \rightarrow \infty$. In our case, this spectrum is

$$
n_{k} \simeq k^{-3 / 2}
$$

Hence as $k \rightarrow \infty$, only the WT component survives. Even $P \simeq 10^{-2} P^{+}$is enough to provide an observable tail in the WT Kolmogorov spectrum.

We should stress out again that at $\lambda=+1$ the picture of turbulence matches the WT prediction both quantitatively and qualitatively. Meanwhile, the spectrum at high $k \mathrm{~s}$ is steeper and closer to the MMT formula. So far we cannot give a consistent explanation of this fact. We can just guess that it is somehow connected with quasisolitons. As an illustration, Fig. 29 shows the conservative evolution of the initial quasisoliton (8.13) with parameter $q / k_{m}=0.1$, which is small enough to justify the Taylor expansion used in its derivation. As expected, we observe that the solution propagates and persists over a relatively long time. This similarity between quasisolitons and real solitons is verified even better in Figs. 30-33 where two initial quasisolitons with $q / k_{m}=0.2$ for the smaller one and $q / k_{m}=0.25$ for the bigger one collide almost elastically. Note here that the solution with smaller amplitude moves with a greater velocity.

\section{Numerical results for $\beta=+3$ and $\lambda=+1$}

Another series of experiments has been performed for the case $\beta=+3$ and $\lambda=+1$. This case is especially attractive due to the fact that the intensity of interaction grows with characteristic wave number in Fourier space 


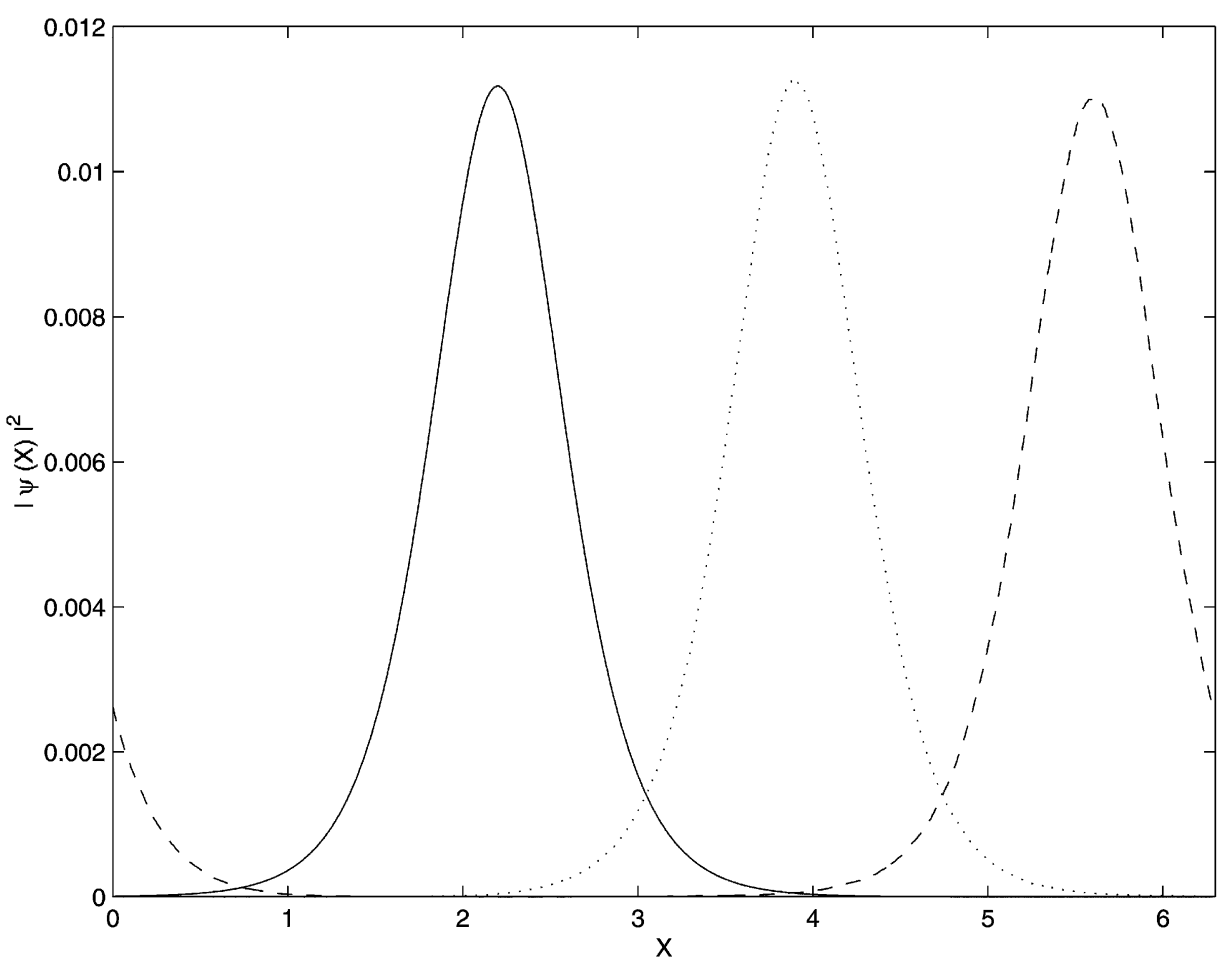

Fig. 29. $\beta=0, \lambda=+1$. Evolution of the initial quasisoliton for $q / k_{m}=0.1$. Solid line $t=0$, dotted line $t=1250$, dashed line $t=2500$.

and one can expect reduced "frozen" turbulence effects compared to the case $\beta=0$. Another motivation is the fact that the scaling of the interaction kernel reproduces the kernel for gravity water waves. Therefore, Eq. (12.1) with $\alpha=\frac{1}{2}, \beta=+3$ can be considered as a model of turbulence of the ocean surface.

The numerical simulation of Eq. (12.1) was performed on a grid of 2048 points in the real space domain of length $2 \pi$. Parameters of the forcing are defined by

$$
F(k)= \begin{cases}0.001 & \text { if } 30<k<42 \\ 0 & \text { otherwise }\end{cases}
$$

and parameters of damping in the "hyperviscosity" form by

$$
D(k)= \begin{cases}-0.05(k-4)^{8} & \text { if } 0<k<4 \\ -0.1(k-824)^{2} & \text { if } 824<k<1024 \\ 0 & \text { otherwise }\end{cases}
$$

Aliasing effects were not of concern due to the run-time control of the fastness of the spectrum decay toward high wave numbers.

The time-step of integration was equal to $\frac{1}{50}$ of the inverse fastest linear frequency in the problem. Such a small value was chosen due to the fact that the time dependence of the individual Fourier harmonics corresponding to intermediate range wave numbers showed the presence of processes of time scale smaller than the smallest linear 


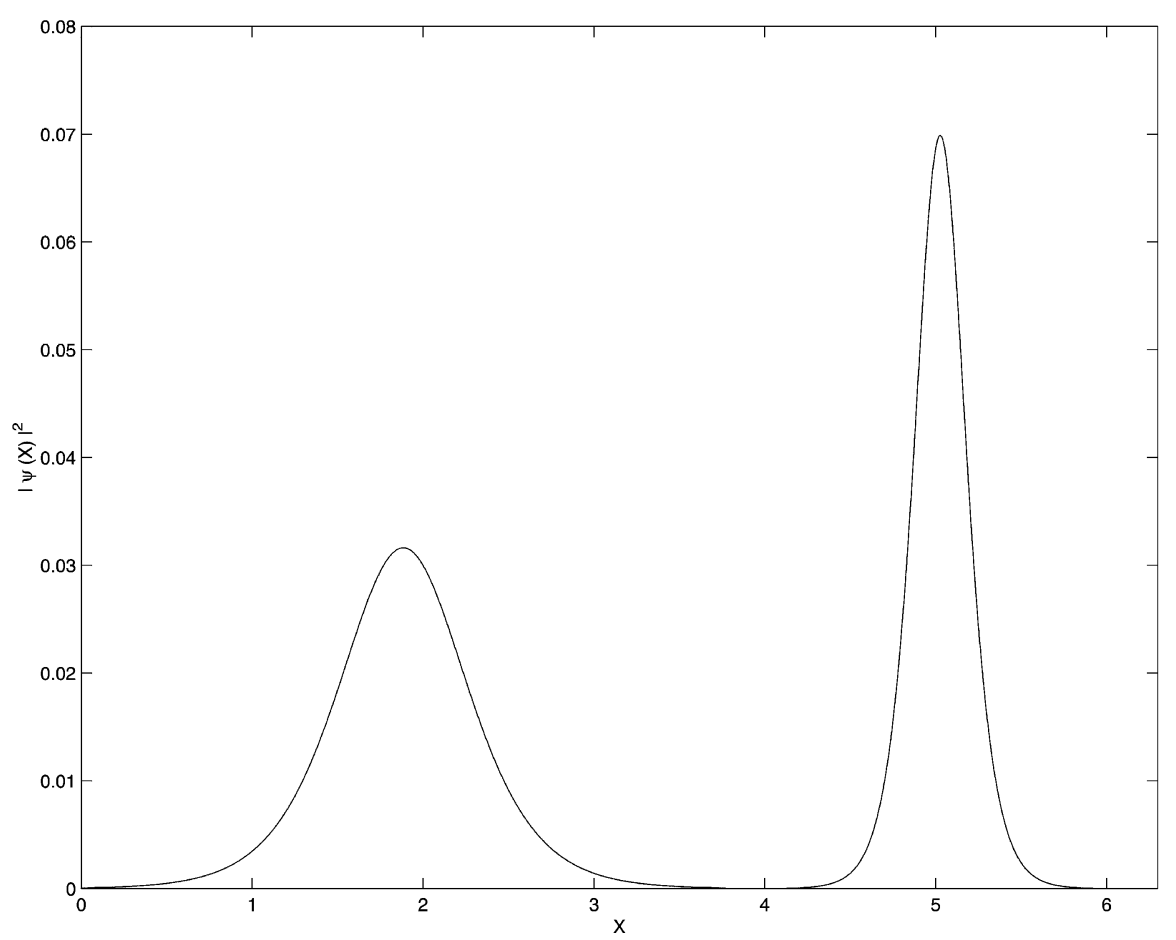

Fig. 30. $\beta=0, \lambda=+1$. Interaction of two initial quasisolitons at $t=0$. The smaller and bigger ones correspond to $q / k_{m}=0.2$ and 0.25 , respectively.

time in the system. This observation was an initial indication of the significant role of nonlinearity in the problem under consideration.

Eq. (12.1) was integrated numerically over long times for different kinds of initial conditions: low level random noise and single harmonic excitation $(k=30)$ initial conditions. While initial stages of computations were quantitatively different, the later stages of evolution were strikingly similar. Starting from big enough times, the wave system was separated into several soliton-like moving structures and low-amplitude quasi-linear waves. Processes of interaction of solitons and waves slowly redistributed the number of waves in a way leading to the growth of initially bigger solitons and the collapse of initially smaller solitons. Finally, the system was clearly separated into a state with one moving soliton and quasi-linear waves.

We interpret the observed phenomenon as similar to the "droplet" effect observed earlier in non-integrable NLS equation [20]. The soliton solution turns out to be the statistical attractor for nonlinear non-integrable wave systems: long time evolution leads to the condensation of the integral of total number of waves into the single soliton which minimizes the Hamiltonian.

Figs. 34 and 35 show snapshots of the final state of the system: the single soliton is moving with constant speed on the background of quasi-linear waves. A quantitative comparison shows that the parameters of the observed object are close to the parameters of the quasisoliton solution (8.13).

One should emphasize that there is a difference between the situation observed in the present work and former observations of "droplet" effects in non-integrable NLS equations. Solitons observed in [20] were exact stable solutions of the corresponding NLS equation. Solitary solutions observed in the present work are "quasisolitons" which are unstable at least in a certain range of parameters. 


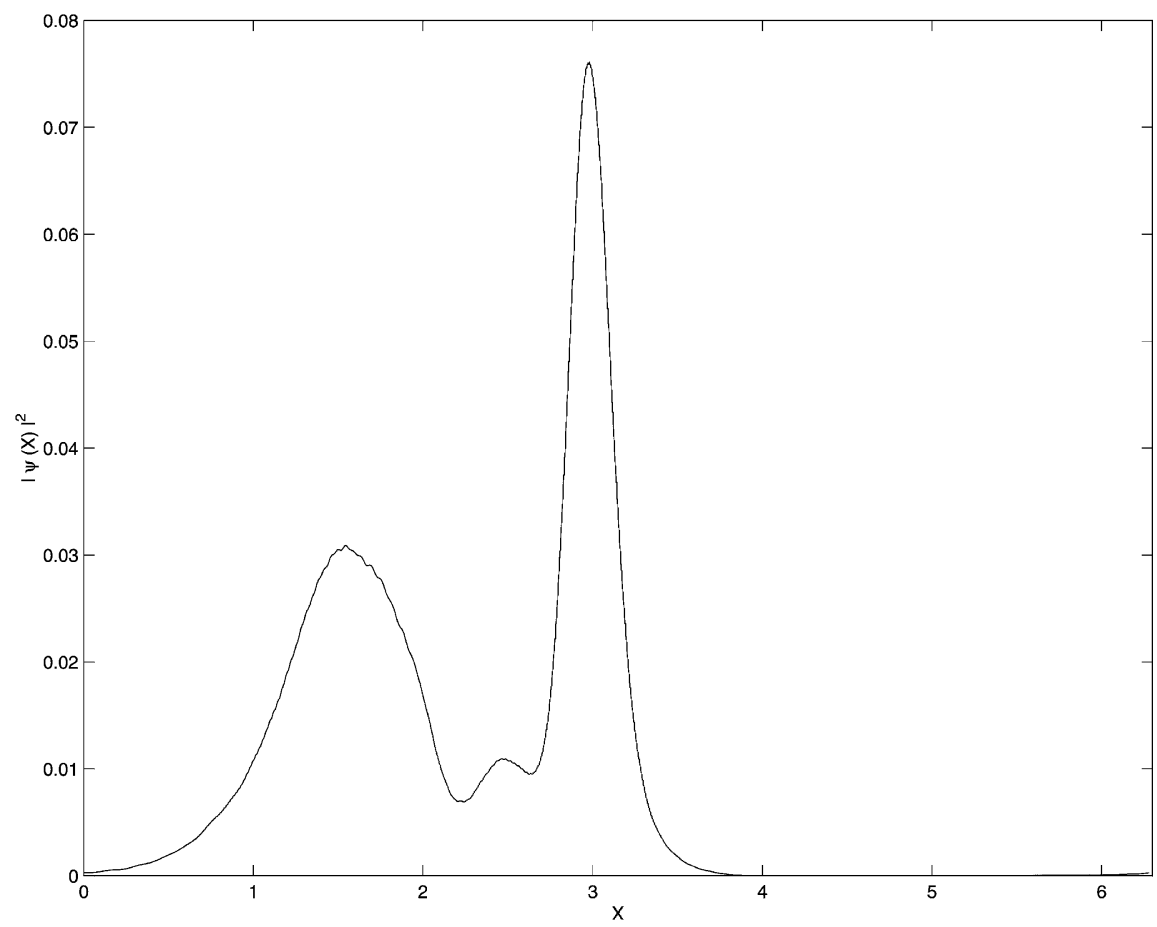

Fig. 31. $\beta=0, \lambda=+1$. Interaction of two initial quasisolitons at $t=37.5$.

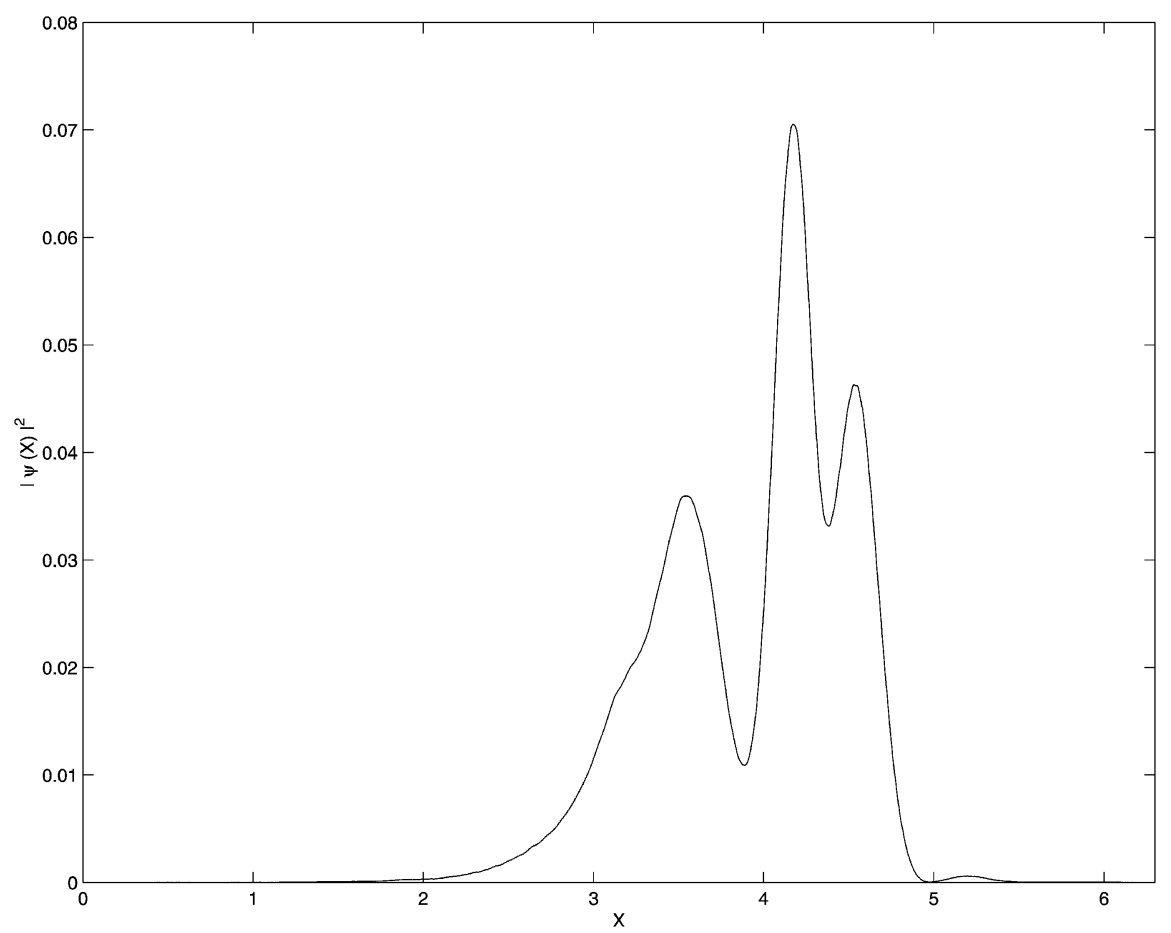

Fig. 32. $\beta=0, \lambda=+1$. Interaction of two initial quasisolitons at $t=50$. 


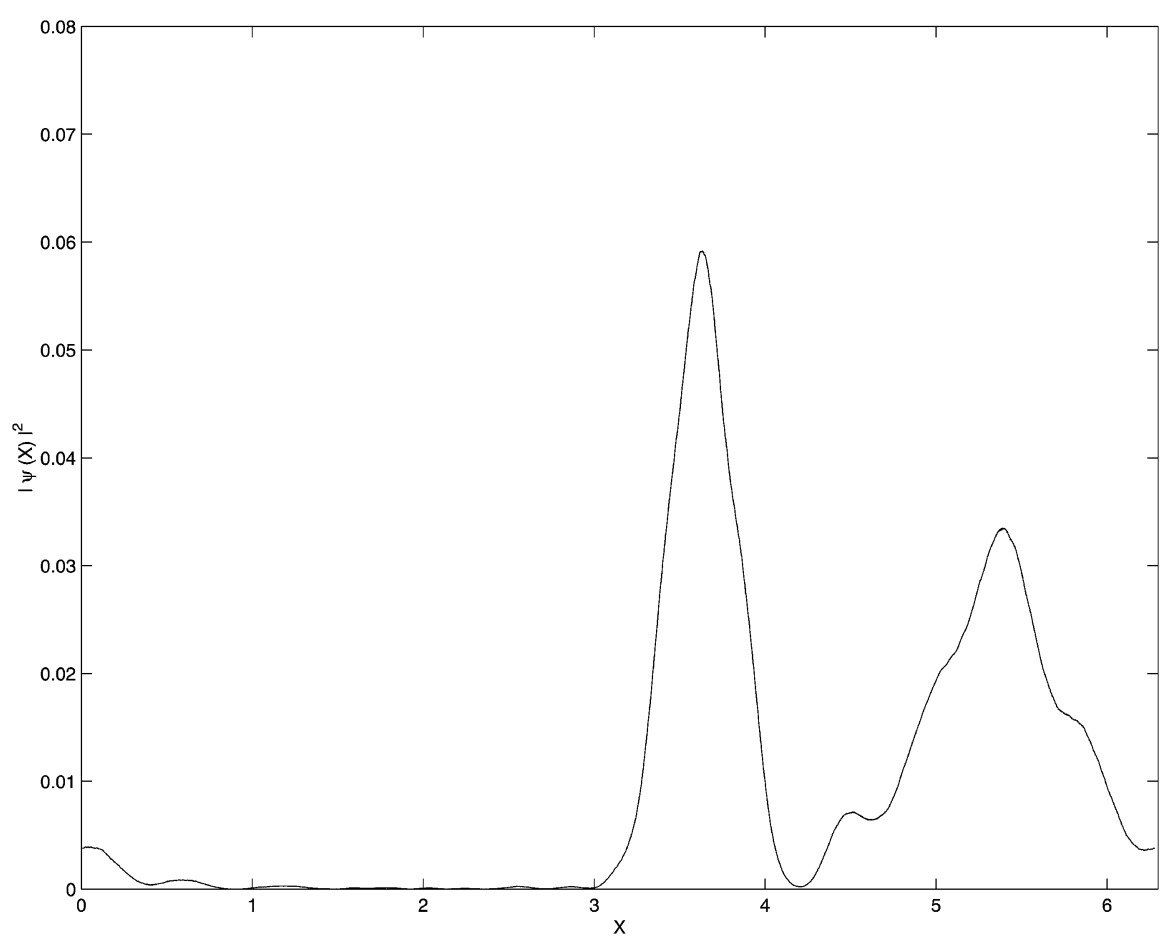

Fig. 33. $\beta=0, \lambda=+1$. Interaction of two initial quasisolitons at $t=100$.

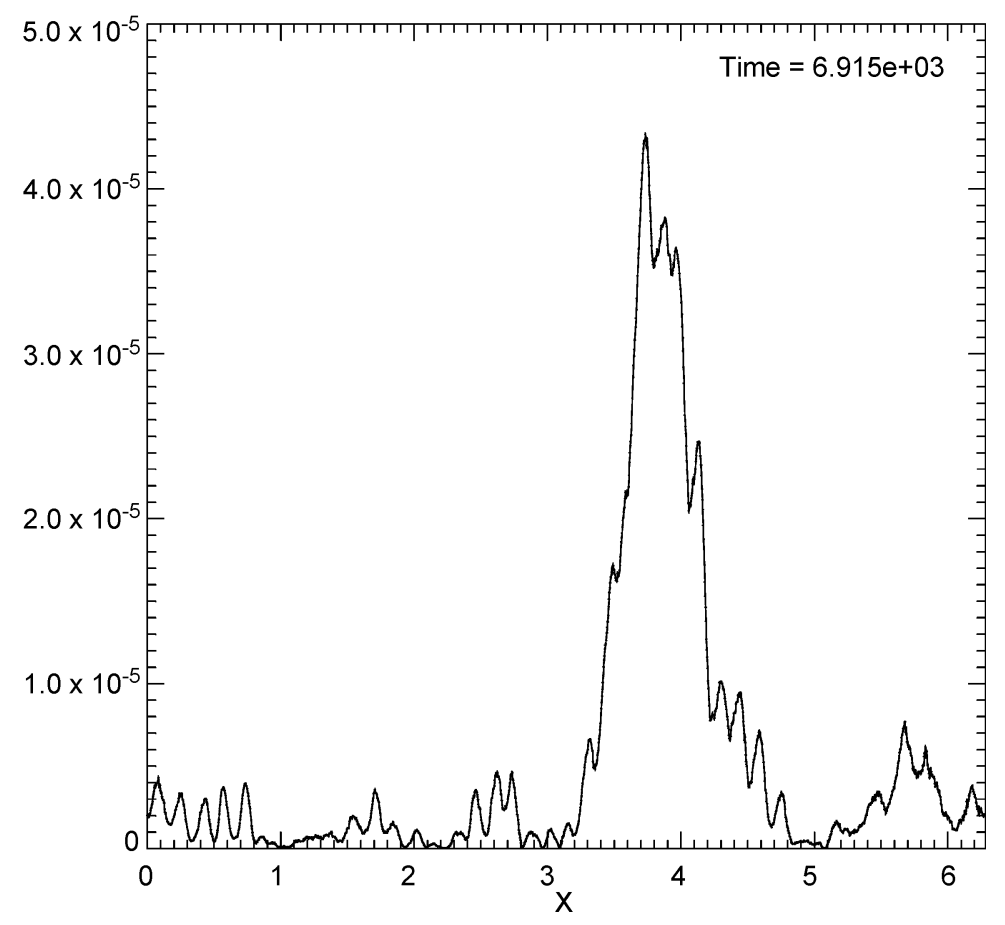

Fig. 34. $\beta=3, \lambda=+1$. Single moving soliton, $t=6915$. 


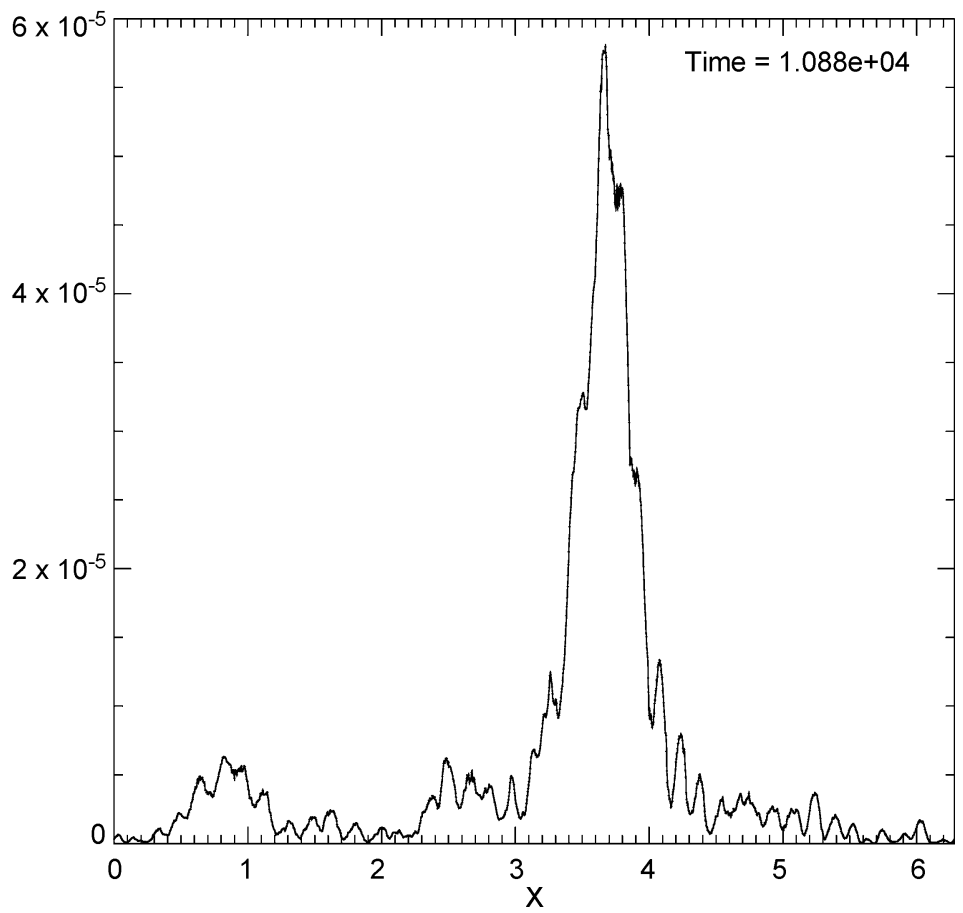

Fig. 35. $\beta=3, \lambda=+1$. Single moving soliton, $t=10880$.

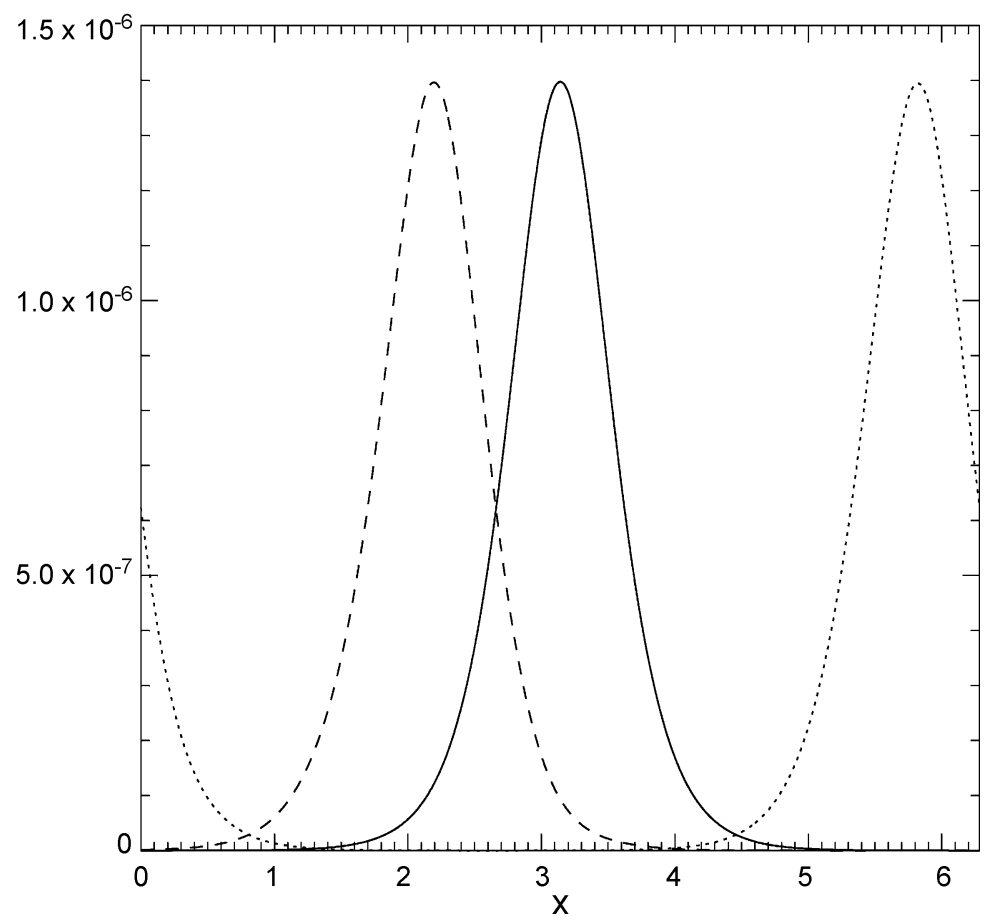

Fig. 36. $\beta=3, \lambda=+1$. Evolution of the initial quasisoliton for $q / k_{m}=0.1$. Solid line $t=0$, dotted line $t=23.6$, dashed line $t=47.1$. 


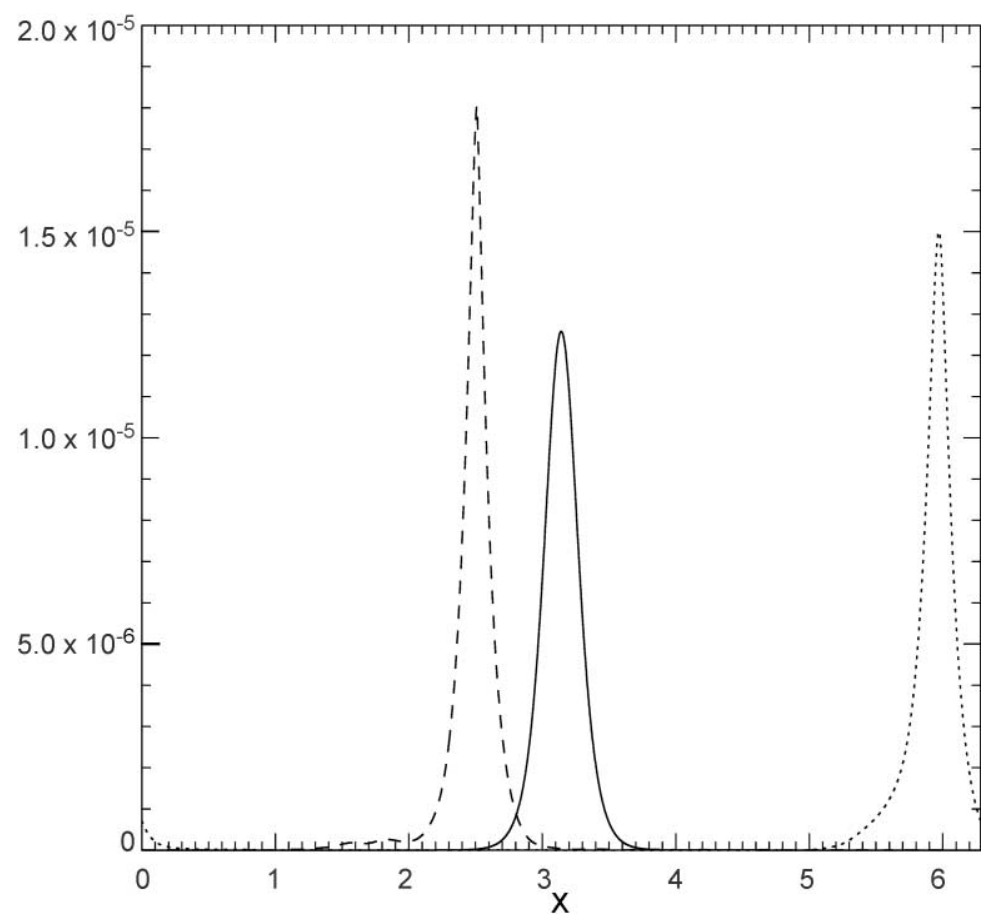

Fig. 37. $\beta=3, \lambda=+1$. Evolution of the initial quasisoliton for $q / k_{m}=0.3$. Solid line $t=0$, dotted line $t=23.6$, dashed line $t=47.1$.

In Fig. 36 the initial condition is the quasisoliton (8.13) with parameter $q / k_{m}=0.1$. Here again, it behaves as the soliton should: it moves without any detectable change of shape. Fig. 37 shows the evolution for $q / k_{m}=0.3$. One can interpret such initial condition as a "deformed" quasisoliton. This initial condition rapidly develops moving singularity collapsing, presumably, in finite time.

\section{Conclusion}

The MMT model with $\alpha<1$ and either sign of nonlinearity exhibits coherent structures. In the case of negative nonlinearity these structures are weak collapses. These collapses are a powerful mechanism of energy dissipation, which dominates in all our numerical experiments. Weak turbulence coexists with collapses, and is responsible for the formation of Kolmogorov-type tails of wave spectra. But it carries to high wave numbers just a small part of the energy (less than 5\%).

One may hope to get "pure" WT by decreasing the level of nonlinearity. But to achieve an adequate modeling of the continuous medium, one should take a very fine mesh (at least $10^{4}$ harmonics) and apply forcing in a broad range (say $10<k<100$ ). Otherwise effects of "frozen turbulence" will blur the picture. Such experiments would be very time consuming.

The case of positive nonlinearity is less clear. In this case the picture of turbulence is qualitatively similar to WT, but the slope of the spectrum fits better the MMT spectrum. So far we do not have a satisfactory explanation of this phenomenon. Probably it could be explained by the presence of interacting quasisolitons. In this case again, experiments with a larger number of harmonics could give a result closer to WT predictions. 
The relative "suppression" of WT in the MMT model can be explained by a peculiarity of the resonant conditions. In the one-dimensional case with $\alpha=\frac{1}{2}$, only well-separated waves interact. Indeed, one can see from (2.10) that

$$
\left|\frac{k_{2}}{k_{1}}\right|=\left(\frac{1}{\xi}+1+\xi\right)^{2}, \quad \xi>0,
$$

and therefore $\min \left|k_{2} / k_{1}\right|=9$ is reached at $\xi=1$. This phenomenon can be called "sparsity of resonances". Due to this sparsity, four-wave resonances easily lose the competition with coherent structures - collapses and quasisolitons. In this sense the MMT model is not an optimal object for checking the validity of WT theory. We can offer the following model, which includes the interaction of two types of waves

$$
\begin{aligned}
H= & \int|k|^{\alpha}\left(\left|a_{k}\right|^{2}+s\left|b_{k}\right|^{2}\right) \mathrm{d} k+\int\left|k k_{1} k_{2} k_{3}\right|^{\beta / 4}\left(a_{k}^{*} a_{1}^{*} a_{2} a_{3}+2 p_{1} a_{k}^{*} b_{1}^{*} a_{2} b_{3}+p_{2} b_{k}^{*} b_{1}^{*} b_{2} b_{3}\right) \\
& \times \delta\left(k+k_{1}-k_{2}-k_{3}\right) \mathrm{d} k \mathrm{~d} k_{1} \mathrm{~d} k_{2} \mathrm{~d} k_{3} .
\end{aligned}
$$

If $\alpha>1$ and $\beta<2 \alpha-1$, the corresponding dynamical system does not describe any coherent structures which could compete with four-wave resonances. Meanwhile, for $s \neq 1$, it describes non-trivial resonant interactions for different waves propagating in the same direction. The system (15.2) looks like a possible object for the simulation of wave turbulence. In the special case $\alpha=2$ and $\beta=0$, it describes coupled NLS equations.

\section{Acknowledgements}

The authors are grateful to D. McLaughlin, A. Majda, D. Cai and E. Tabak for useful discussions and for providing their results before publication. This work was performed in the framework of the NATO Linkage Grant OUTR.LG 970583. V. Zakharov and A. Pushkarev also acknowledge support of the US Army, under the grant DACA 39-99-C-0018, and of ONR, under the grant N00014-98-1-0439. F. Dias and P. Guyenne acknowledge support of

DGA, under the contract ERS 981135. A. Pushkarev wishes to thank F. Dias for his hospitality during a visit to Nice in the fall of 1998.

\section{References}

[1] R.E. Peierls, Zur kinetischen Theorie der Wärmeleitungen in Kristallen, Ann. Phys. 3 (1929) 1055-1101.

[2] K. Hasselmann, On the non-linear energy transfer in a gravity-wave spectrum. Part 1. General theory, J. Fluid Mech. 12 (1962) 481-500.

[3] K. Hasselmann, On the non-linear energy transfer in a gravity-wave spectrum. Part 2. Conservative theorems, wave-particle analogy, irreversibility, J. Fluid Mech. 15 (1963) 273-281.

[4] V.E. Zakharov, Stability of periodic waves of finite amplitude on a surface of deep fluid, J. Appl. Mech. Tech. Phys. 9 (1968) 190-194.

[5] A.N. Pushkarev, V.E. Zakharov, Turbulence of capillary waves, Phys. Rev. Lett. 76 (1996) 3320-3323.

[6] V.E. Zakharov, N.N. Filonenko, Weak turbulence of capillary waves, J. Appl. Mech. Tech. Phys. 4 (1967) 506-515.

[7] A.J. Majda, D.W. McLaughlin, E.G. Tabak, A one-dimensional model for dispersive wave turbulence, J. Nonlinear Sci. 6 (1997) 9-44.

[8] D. Cai, A.J. Majda, D.W. McLaughlin, E.G. Tabak, Spectral bifurcations in dispersive wave turbulence, Proc. Natl. Acad. Sci. 96 (1999) 14216-14221.

[9] D. Cai, D. McLaughlin, A. Majda, E. Tabak, Dispersive wave turbulence in one dimension, Physica D 152-153 (2001) 551-572.

[10] D. Cai, D. McLaughlin, Chaotic and turbulent behavior of unstable one-dimensional nonlinear dispersive waves, J. Math. Phys. 41 (2000) 4125-4153.

[11] V.E. Zakharov, Statistical theory of gravity and capillary waves on the surface of a finite-depth fluid, Eur. J. Mech. B 18 (1999) 327-344.

[12] G. Falkovich, Bottleneck phenomenon in developed turbulence, Phys. Fluids 6 (1994) 1411-1414.

[13] A.N. Pushkarev, On the Kolmogorov and frozen turbulence in numerical simulation of capillary waves, Eur. J. Mech. B 18 (1999) $345-352$.

[14] V.E. Zakharov, E.A. Kuznetsov, Optical solitons and quasisolitons, ZhETF (JETP) 113 (1998) 1892-1913. 
[15] S. Dyachenko, A.C. Newell, A.N. Pushkarev, V.E. Zakharov, Optical turbulence: weak turbulence, condensates and collapsing filaments in the nonlinear Schrödinger equation, Physica D 57 (1992) 96-160.

[16] V.E. Zakharov, V. Lvov, G. Falkovich, Kolmogorov Spectra of Turbulence, Vol. I, Springer, Berlin, 1992.

[17] A.M. Balk, V.E. Zakharov, Stability of weak turbulence Kolmogorov spectra, Am. Math. Soc. Transl. Ser. 2 182 (1998) 31-81.

[18] E.A. Kuznetsov, A.M. Rubenchik, V.E. Zakharov, Soliton stability in plasmas and hydrodynamics, Phys. Rep. 142 (1986) $103-165$.

[19] O.M. Phillips, The Dynamics of the Upper Ocean, Cambridge University Press, Cambridge, 1977.

[20] V.E. Zakharov, A.N. Pushkarev, V.F. Shvets, V.V. Yan'kov, Soliton turbulence, JETP Lett. 48 (1988) 83-87. 